DISSERTAÇÃO DE MESTRADO

ON THE PERFORMANCE OF

VIDEO QUALITY ASSESSMENT METHODS FOR DIFFERENT SPATIAL AND TEMPORAL RESOLUTIONS

Welington Yorihiko Lima Akamine

UNIVERSIDADE DE BRASÍLIA 


\section{UNIVERSIDADE DE BRASÍLIA \\ FACULDADE DE TECNOLOGIA \\ DEPARTAMENTO DE ENGENHARIA ELÉTRICA}

\section{ON THE PERFORMANCE OF VIDEO QUALITY METRICS FOR DIFFERENT RESOLUTIONS}

\section{WELINGTON YORIHIKO LIMA AKAMINE}

DISSERTAÇÃO DE MESTRADO SUBMETIDA AO DEPARTAMENTO DE ENGENHARIA ELÉTRICA DA FACULDADE DE TECNOLOGIA DA UNIVERSIDADE DE BRASÍLIA, COMO PARTE DOS REQUISITOS NECESSÁRIOS PARA A OBTENÇÃO DO GRAU DE MESTRE.

APROVADA POR:

Myken forion

MYLÉNE CHRISTIINE QUEIROZ DE FARIAS , Dra., ENE/UNB

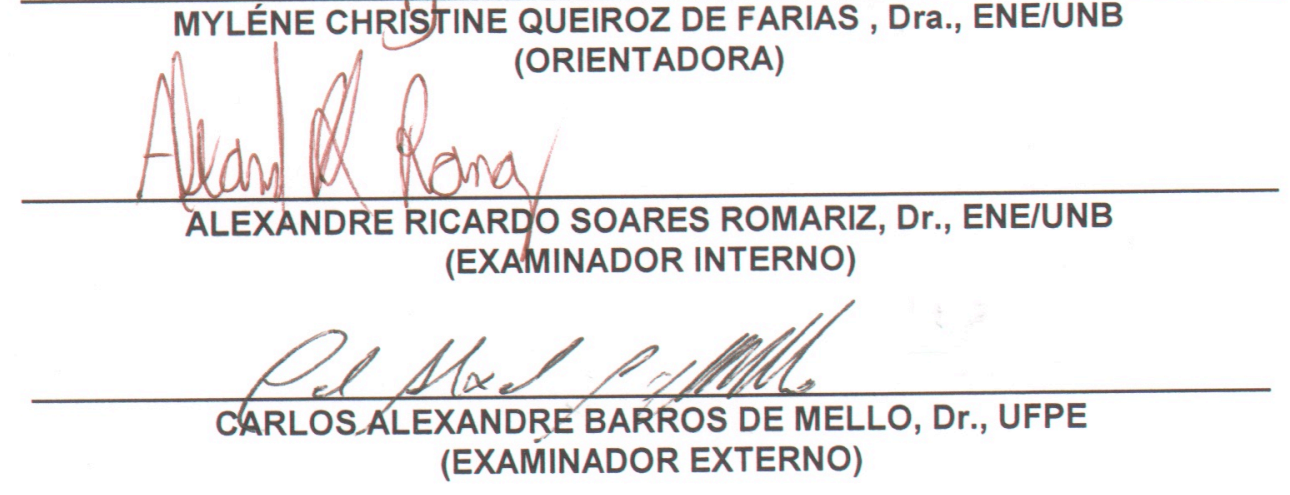

Brasília, 16 de fevereiro de 2017. 
UNIVERSIDADE DE BRASÍLIA

Faculdade de Tecnologia

\title{
DISSERTAÇÃO DE MESTRADO \\ ON THE PERFORMANCE OF VIDEO QUALITY ASSESSMENT METHODS FOR DIFFERENT SPATIAL AND TEMPORAL RESOLUTIONS
}

\author{
Welington Yorihiko Lima Akamine \\ Dissertação de Mestrado submetida ao Departamento de Engenharia \\ Elétrica como requisito parcial para obtenção \\ do grau de Mestre em Engenharia de Sistemas Eletrônicos e Automação
}

Banca Examinadora

Prof. Mylène Christine Queiroz de Farias, Ph.D,

ENE/UnB

Orientadora

Prof. Alexandre Ricardo Soares Romariz, Ph.D,

$\mathrm{ENE} / \mathrm{UnB}$

Examinador interno

Prof. Carlos Alexandre Barros de Mello, Ph.D,

$\mathrm{CIn} / \mathrm{UFPE}$

Examinador externo 
FICHA CATALOGRÁFICA

AKAMINE, WELINGTON YORIHIKO LIMA

ON THE PERFORMANCE OF VIDEO QUALITY ASSESSMENT METHODS FOR DIFFERENT SPATIAL AND TEMPORAL RESOLUTIONS [Distrito Federal] 2017.

xvi, 78 p., 210 x 297 mm (ENE/FT/UnB, Mestre, Engenharia Elétrica, 2017).

Dissertação de Mestrado - Universidade de Brasília, Faculdade de Tecnologia.

Departamento de Engenharia Elétrica

1. Video Quality Assessment Methods $\quad$ 2. Spatial Resolution

3. Temporal Resolution

4. Runtime Performance

I. ENE/FT/UnB

II. Título (série)

\section{REFERÊNCIA BIBLIOGRÁFICA}

AKAMINE, W. Y. L. (2017). ON THE PERFORMANCE OF VIDEO QUALITY ASSESSMENT

METHODS FOR DIFFERENT SPATIAL AND TEMPORAL RESOLUTIONS . Dissertação de Mestrado, Publicação: PGEA.DM - 655/2017, Departamento de Engenharia Elétrica, Universidade de Brasília, Brasília, DF, 78 p.

\section{CESSÃO DE DIREITOS}

AUTOR: Welington Yorihiko Lima Akamine

TÍTULO: ON THE PERFORMANCE OF VIDEO QUALITY ASSESSMENT METHODS FOR DIFFERENT SPATIAL AND TEMPORAL RESOLUTIONS .

GRAU: Mestre em Engenharia de Sistemas Eletrônicos e Automação $\quad$ ANO: 2017

É concedida à Universidade de Brasília permissão para reproduzir cópias desta Dissertação de Mestrado e para emprestar ou vender tais cópias somente para propósitos acadêmicos e científicos. Os autores reservam outros direitos de publicação e nenhuma parte dessa Dissertação de Mestrado pode ser reproduzida sem autorização por escrito dos autores.

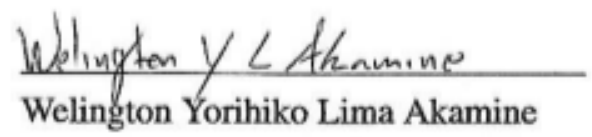

Depto. de Engenharia Elétrica (ENE) - FT

Universidade de Brasília (UnB)

Campus Darcy Ribeiro

CEP 70919-970 - Brasília - DF - Brasil 


\section{Acknowledgments}

I would first like to acknowledge Mylène Farias, my tutor, for her patience, encouragement, and immense knowledge. Knowing that I could always count on her help and guidance gave me the strength necessary to face the challenges I faced during this research. I could not be more grateful to have had her as my tutor.

I am grateful to my parents, Mercedes and Ruy, for their unconditional love, help and support. And my sisters, Aline, Larissa, and Alicia, for always being there, as friends, supporting me during all these years.

Also, I will be eternally thankful for the assistance given by Pedro Garcia. His valuable and constructive suggestions were essential during the development of this research work.

Finally, I thank all my colleagues and friends in the Digital Signal Processing Group (GPDS). Gustavo and Johnathan, with whom I shared an office at UnB. Dario, who I met in my first day as a graduate student and to whom I am thankful for his friendship during this journey. Helard and Alexandre, for helping me understand some of the algorithms used in this work.

Welington Yorihiko Lima Akamine 
O consumo de vídeos digitais cresce a cada ano. Vários países já utilizam tv digital e o tráfego de dados de vídeos na internet equivale a mais de $60 \%$ de todo o tráfego de dados na internet. Esse aumento no consumo de vídeos digitais exige métodos computacionais viáveis para o cálculo da qualidade do vídeo. Métodos objetivos de qualidade de vídeo são algoritmos que calculam a qualidade do vídeo. As mais recentes métricas de qualidade de vídeo, apesar de adequadas possuem um tempo de execução alto. Em geral, os algoritmos utilizados são complexos e extraem características espaciais e temporais dos vídeos.

Neste trabalho, realizamos uma análise dos efeitos da redução da resolução espacial no desempenho dos métodos de avaliação da qualidade do vídeo. Com base nesta análise, nós propomos um framework, para a avaliação da qualidade de vídeo que melhora o tempo de execução das métricas objetivas de qualidade de vídeo sem reduzir o desempenho na predição da qualidade do vídeo. O framework consiste em quatro etapas. A primeira etapa, classificação, identifica os vídeos mais sensíveis à redução da resolução espacial. A segunda etapa, redução, reduz a resolução espacial do vídeo de acordo com a distorção presente. A terceira etapa, predição de qualidade, utiliza uma métrica objetiva para obter uma estimativa da qualidade do vídeo. Finalmente, a quarta etapa realiza um ajuste dos índices de qualidade preditos.

Dois classificadores de vídeo são propostos para a etapa de classificação do framework. O primeiro é um classificador com referência, que realiza medidas da atividade espacial dos vídeos. O segundo é um classificador sem-referência, que realiza medidas de entropia espacial e espectral, utilizando Support Vector Machine, para classificar os vídeos. Os classificadores de vídeo têm o objetivo de selecionar o melhor fator de redução da resolução espacial do vídeo. Testamos o framework proposto com 6 métricas objetivas de qualidade de vídeo e 4 bancos de qualidade de vídeo. Com isso, melhoramos o tempo de execução de todas as métricas de qualidade de vídeo testadas.

Também analisamos os efeitos da redução da resolução temporal no desempenho das métricas de qualidade de vídeo. A análise mostra que as métricas de qualidade de vídeo, cujas medidas se baseiam nas características temporais do vídeo, são mais sensíveis à redução temporal. Da mesma forma, vídeos distorcidos por distorções temporais (e.g. perda de pacotes) são mais sensíveis à uma redução temporal. 
The consumption of digital videos increases every year. In addition to the fact that many countries already use digital TV, currently the traffic of internet video services are more than $60 \%$ of the total internet traffic. The growth of digital video consumption demands a viable method to measure the video quality. Objective video quality assessment methods are algorithms that estimates video quality. Recent quality assessment methods provide quality predictions that are well correlated with the subjective quality scores. However, most of these methods are very complex and takes long periods to compute.

In this work, we analyze the effects of reducing the video spatial resolution on the performance of video quality assessment methods. Based on this analysis, we propose a framework for video quality assessment that reduces the runtime performance of a given video quality assessment method without reducing its accuracy performance. The proposed framework is composed of four stages. The first stage, classification, identifies videos that are more sensitive to spatial resolution reduction. The second stage, reduction, aims to reduce the video spatial resolution according to the video distortion. The third stage, quality prediction, estimates the video quality using an objective video quality assessment method. Finally, the fourth stage normalizes the predicted quality scores according to the video spatial resolution.

We design two video classifiers for the first stage of the framework. The first classifier is a full-reference classifier based on a video spatial activity measure. The second is a no-reference classifier based on spatial and spectral entropy measures, which uses a Support Vector Machine (SVM) algorithm. We use the video classifiers to identify the type of distortion in the video and choose the most appropriate spatial resolution. We test the framework using six different video quality assessment methods and four different video quality databases. Results show that the proposed framework improves the average runtime performance of all video quality assessment methods tested.

We also analyze the effects of a temporal resolution reduction on the performance of video quality assessment methods. The analysis shows that video quality assessment methods based on temporal features are more sensitive to temporal resolution reduction. Also, videos with temporal distortions, like packet loss, are very sensitive to temporal resolution reduction. 
1 INTRODUCTION $\ldots \ldots \ldots \ldots \ldots \ldots \ldots \ldots \ldots \ldots \ldots \ldots \ldots \ldots \ldots \ldots \ldots \ldots \ldots \ldots, 1$

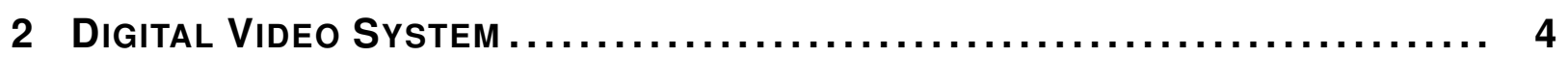

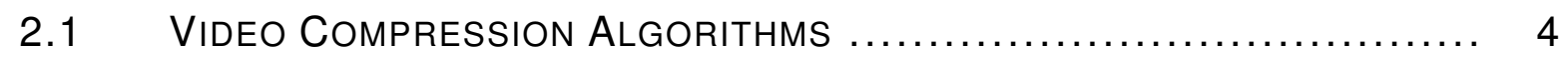

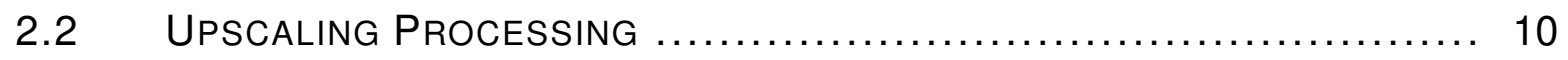

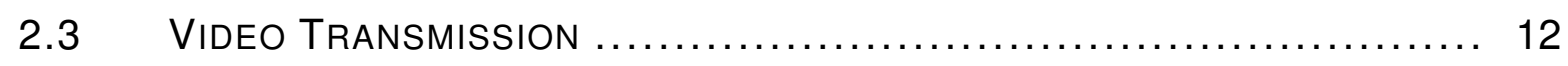

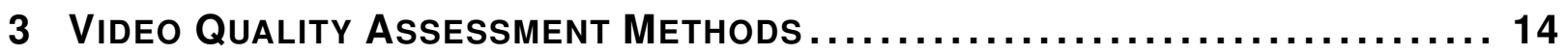

3.1 ObJective Quality Assessment Methods $\ldots \ldots \ldots \ldots \ldots \ldots \ldots \ldots \ldots \ldots \ldots \ldots \ldots \ldots$

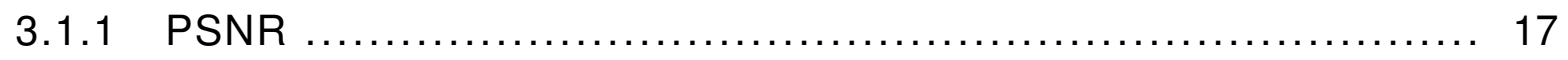

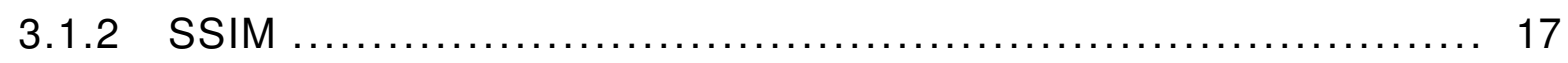

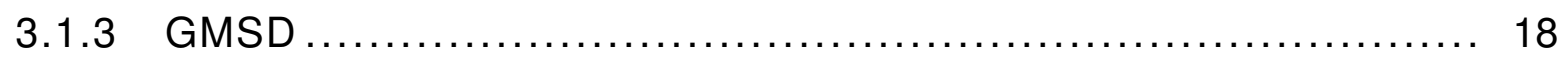

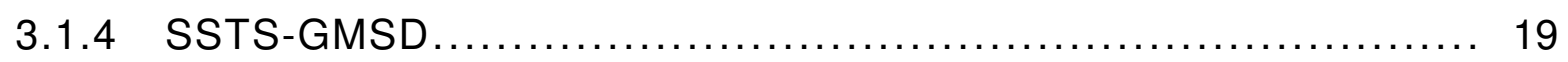

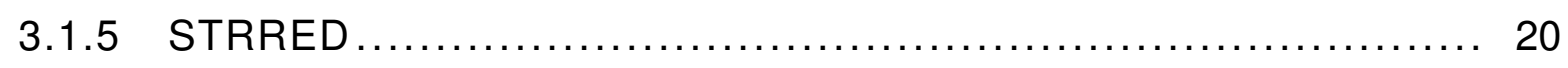

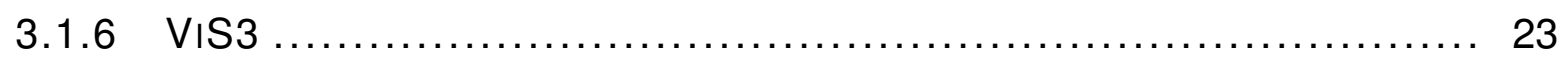

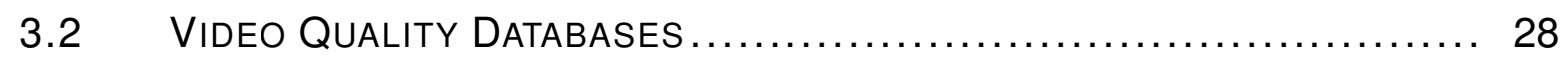

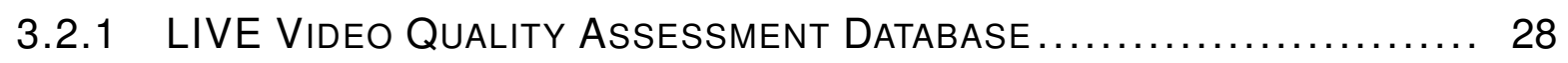

3.2 .2 CSIQ VIDEO QualitY DATABASE ............................... 30

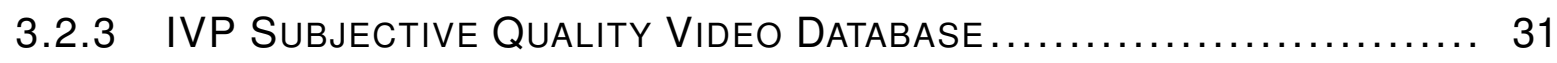

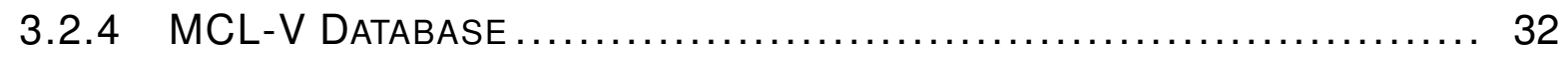

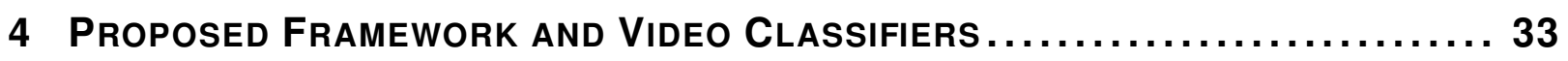

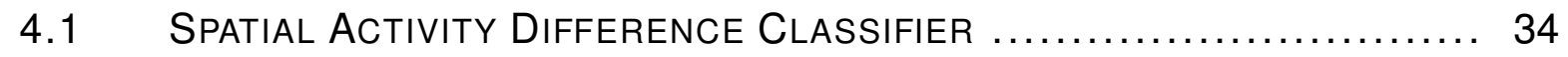

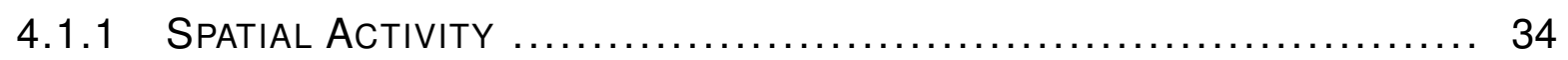

4.1 .2 Spatial Activity Difference Algorithm ........................ 36

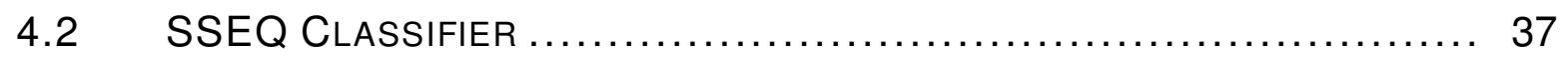

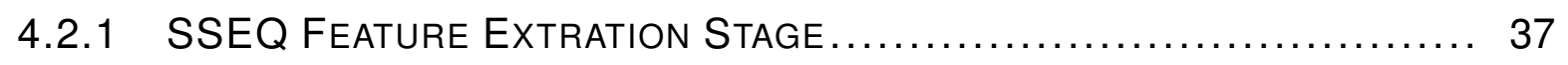

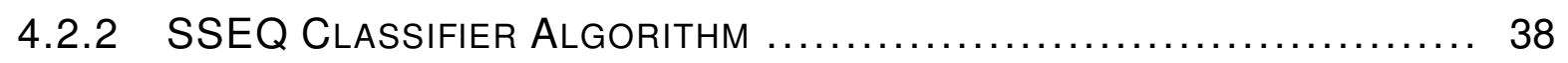

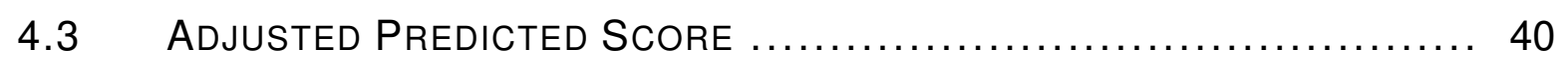

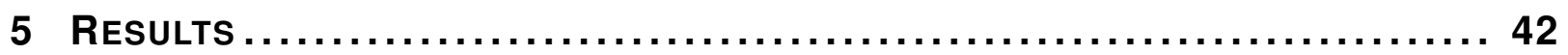

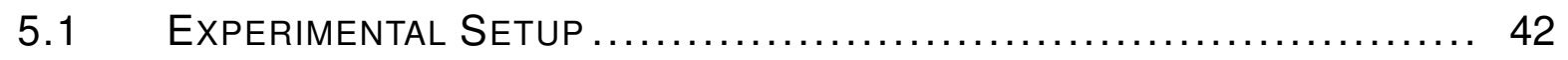

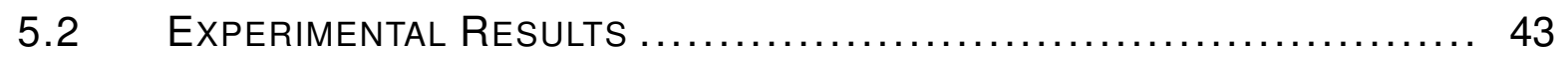

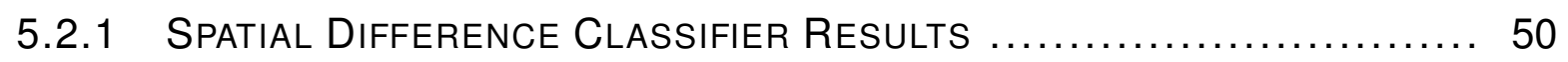

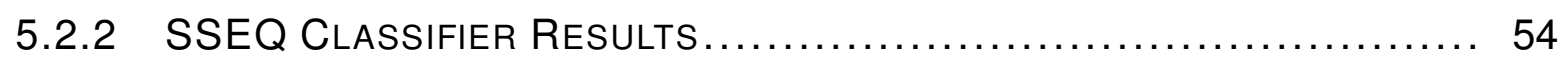

5.2.3 Influence of Frame Rate in Video Quality Metrics ............. 57

5.2 .4 DISCUSSION................................................... 59 


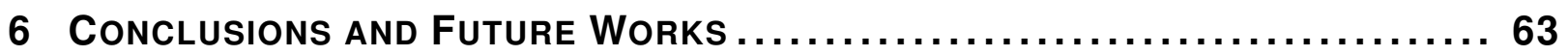

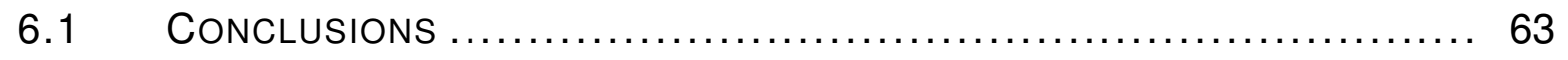

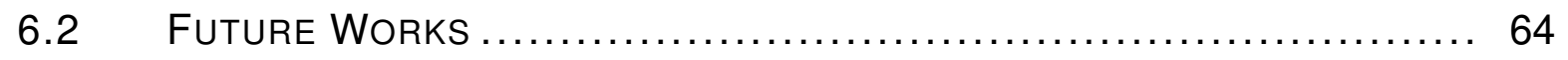

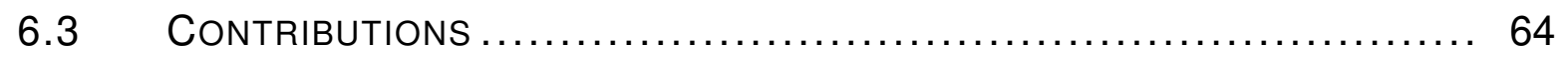

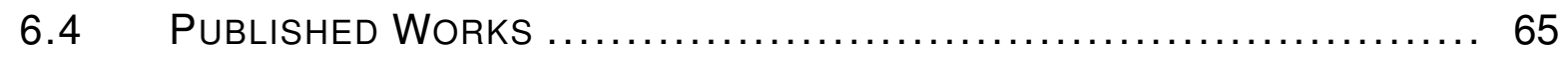

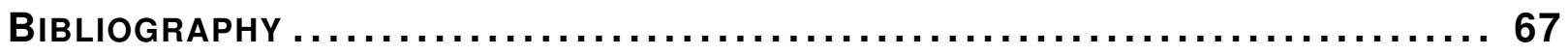

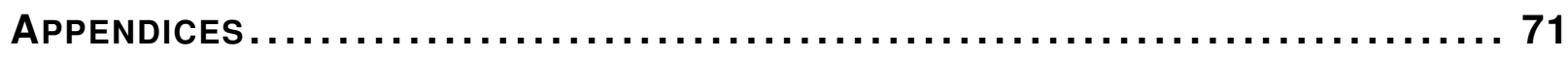

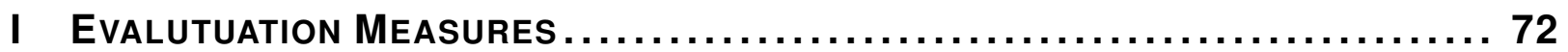

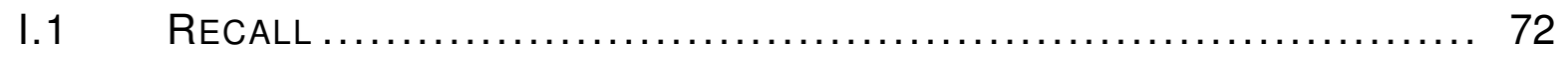

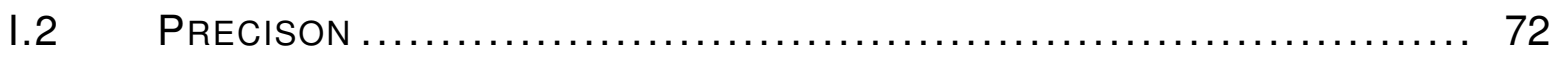

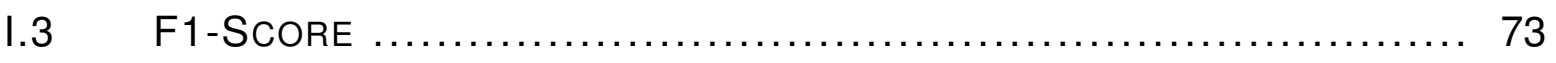

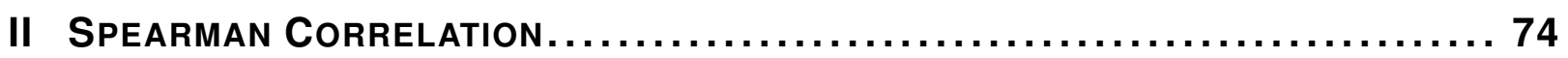




\section{LIST OF FIGURES}

2.1 Overview of Video Transmission Diagram........................................ 4

2.2 Comparison between (a) the reference frame and (b) the frame compressed using a MJPEG algorithm.

2.3 Comparison between (a) the reference frame and (b) the frame compressed using a MPEG-2 compression algorithm.

2.4 Comparison between (a) the reference frame and (b) the frame compressed using an H.264 compression algorithm.

2.5 Comparison between (a) the reference frame and (b) the frame compressed using a DIRAC-Wavelet compression algorithm.

2.6 Comparison between (a) the reference frame and (b) the frame compressed using an H.265 compression algorithm.

2.7 Comparison between (a) the reference frame and (b) the frame compressed by an H.264 algorithm and (c) the frame compressed by an H.264 algorithm with Upscaling.

2.8 Comparison between (a) the reference frame and (b) the frame distorted by white noise.

2.9 Comparison between (a) the reference frame and (b) the frame distorted by packet loss artifacts

3.1 Comparison between the images with the same MSE score, but with different distortions. (a) Original Image. (b) Contrast-stretched image. (c) Mean-shifted image. (d) JPEG compressed image. (e) Blurred image. (f) Salt-pepper impulsive

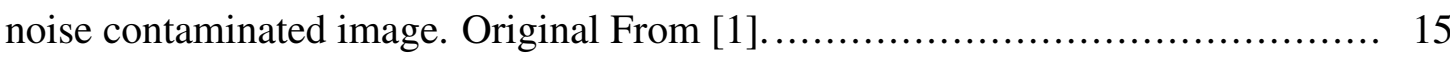

3.2 Diagram of the SSIM Algorithm............................................ 17

3.3 Example of Spatial Temporal Slices. Originals taken from [2]...................... 19

3.4 Diagram of SSTS-GMSD. Original from [2] ................................... 20

3.5 Result of steerable pyramid decomposition in three scales and three orientations. Three sub-bands images at each scale and the final lowpass image. Original from [3]

3.6 Diagram of ViS1 Algorithm. Original from [4] ................................ 23

3.7 Diagram of ViS2 Algorithm. Original from [4] .............................. 26

3.8 Diagram of ViS3 Algorithm. Original from [4] ................................. 28

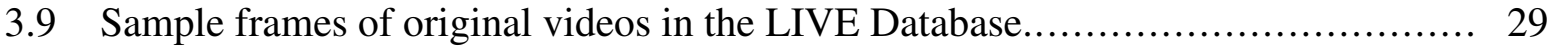

3.10 Sample frames of original videos in the CSIQ Database............................ 30

3.11 Sample frames of original videos in the IVP Database ........................... 31

3.12 Sample frames of original videos in the MCL-V Database. ........................ 32

4.1 High Level Overview of the Downsampling Video Framework. ................... 33 
4.2 Sample frames (unimpaired and distorted) are shown in the left, while their Sobel filtered versions are shown in the right, along with the spatial activity (SA) measure.

4.3 Diagram of the proposed Spatial Activity Classifier. .............................. 36

4.4 Diagram of SSEQ Feature Extraction. .......................................... 37

4.5 SSEQ Video Feature Extraction.................................................. 39

4.6 High-level overview of SSEQ algorithm for traning and testing stages............. 39

4.7 Plot of ViS3 versus Mean Opnion Score. The videos used were from CSIQ Database and they were downsampling to $768 \times 432$ and $384 \times 216 \ldots \ldots \ldots \ldots \ldots \ldots . \ldots 4$

4.8 High Level Overview of the Adjustment of Predicted Score......................... 41

5.1 Box plot of Spearman Correlation Coeficient (SCC) across 1000 simulations, where we ramdonly chose $80 \%$ of the reference videos and their distortions versions. 52

5.2 Box plot of SCC across 1000 test simulations, where we ramdonly chose $20 \%$ of the reference videos and their distortions versions.

5.3 SSIM score of 'Blue Sky' video distorted by H.264 Compression, MPEG-2 Compression and Packet-Loss.

5.4 Spearman correlation coefficient versus computational time (in log space). Comparing the accuracy performance when using the Spatial Activity classifier and the video in their original resolution.

5.5 Spearman correlation coefficient versus computational time (in log space). Comparing the accuracy performance when using the SSEQ classifier and the video in their original resolution. 
4.1 F1 scores using threshold for the Difference in Spatial Activity $(\Delta \mathrm{SA})$, varying

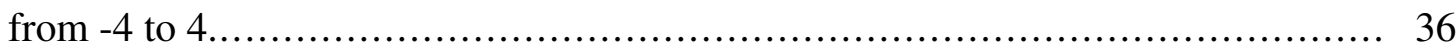

4.2 F1 score of the proposed SSEQ-based classifier for different temporal resolutions. . 39

4.3 Mean Confusion Matrix of proposed SSEQ-based Distortion Classifier, for 1,000 simulations. ............................................................. 40

4.4 Evaluation of the proposed SSEQ-based Distortion Classifier: median values of Precision, Recall, and F1 Score for 1,000 simulations. ......................... 40

5.1 Comparison of Video Quality Databases Specifications. ........................... 43

5.2 Spearman Correlation Coefficient (SCC) of the quality assessment methods, tested on the LIVE database. ............................................................ 44

5.3 Spearman Correlation Coefficient (SCC) of the quality assessment methods, tested on the CSIQ database. ....................................................... 44

5.4 Spearman Correlation Coefficient (SCC) of the quality assessment methods, tested on the IVP database.

5.5 Spearman Correlation Coefficient (SCC) of the quality assessment methods, tested on the MCL-V database.

5.6 Spearman Correlation Coefficient (SCC) of the quality assessment methods, tested on the LIVE database.

5.7 Spearman Correlation Coefficient (SCC) of the quality assessment methods, tested on the CSIQ database.

5.8 Spearman Correlation Coefficient (SCC) of the quality assessment methods, tested on the IVP database.

5.9 Spearman Correlation Coefficient (SCC) of the quality assessment methods, tested on the MCL-V database.

5.10 Spearman Correlation Coefficient (SCC) of the VIS3 metric, tested on all databases. 48

5.11 Spearman Correlation Coefficient (SCC) of the STRRED metric, tested on all databases.

5.12 Spearman Correlation Coefficient (SCC) of the SSTS-GMSD metric, tested on all databases.

5.13 Spearman Correlation Coefficient (SCC) of the GMSD metric, tested on all databases. 49

5.14 Spearman Correlation Coefficient (SCC) of the SSIM metric, tested on all databases. 49

5.15 Spearman Correlation Coefficient (SCC) of the PSNR metric, tested on all databases. 49

5.16 Spearman Correlation Coefficient (SCC) of all objective methods, using the proposed framework with the Spatial Activity Difference Classifier. 
5.17 T-Test results between the SCC of various video quality assessment methods for all databases. The video quality assessment methods evaluate the videos in their original spatial resolution (Ori), 384×216 resolution (Min), using the framework with an ideal classifier (Ideal), and with SA classifier (SA)

5.18 Average running time for performing an objective quality assessment with and without spatial activity classifier (in seconds).

5.19 Median Spearman Correlation Coefficient of all methods using SSEQ Distortion Classifier in 1000 simulations.

5.20 T-Test results between the SCC of various video quality assessment methods for all databases. The video quality assessment methods evaluate the videos in their original spatial resolution (Ori), 384x216 resolution (Min), using the framework with an ideal classifier (Ideal), and with SSEQ classifier (SSEQ).

5.21 Average running time for performing an objective quality assessment with and without SSEQ classifier (in seconds).

5.22 Spearman Coefficient Correlation of the methods when varying the video Frames Per Second (FPS).

5.23 Spearman Coefficient Correlation of the metrics when varying the video FPS for each video distortion.

I.1 Example of a Confusion Matrix 
BBC British Brodcasting Corporation. 7

DCT Discrete Cosine Transform. 5, 8, 37, 38

DFT Discrete Fourier Transform. 23

DMOS Difference Mean Opinion Scores. 29, 31, 49

FPS Frames Per Second. xiii, 29, 31, 32, 39, 54-56, 61, 62

FR Full-Reference. 14, 17

GMSD Gradient Magnitude Similarity Deviation. 16-20, 43-48, 50, 52, 55, 56, 63, 64

GOF Group of Video Frames. 25

GSM Gaussian Scale Mixture. 21, 22

HVS Human Visual System. 5, 15, 16

INLSA Iterated Nested Least-Squares Algorithm. 49

ITU International Telecommunication Union. 14

JND Just Noticeable Differences. 16

MOAVI Monitoring Of Audiovisual Quality by Key Indicators. 36

MOS Mean Opinion Scores. 1, 30, 32, 49

MSE Mean Squared Error. 14, 15, 17, 24

NR No-Reference. 14

PSNR Peak Signal-to-Noise Ratio. 1, 7, 14, 17, 43-45, 48, 50, 52, 54-56, 63, 64

RMS Root Mean Squared. 23, 24, 27, 34

RR Reduced-Reference. 14, 17

RRED Reduced Reference Entropic Differencing. 20, 21

SA Spatial Activity. 34, 36 
SAMVIQ Subjective Assessment Methodology for Video Quality. 30

SCC Spearman Correlation Coeficient. xi, 43, 51-54, 74

SRRED Spatial Reduced Reference Entropic Differences. 21, 22

SSEQ Spatial-Spectral Entropy-based Quality. 37-40, 54, 64

SSIM Structural Similarity Index. 1, 16-18, 43-45, 48, 50-52, 55, 56, 63, 64

SSTS-GMSD Spatial and Spatial-Temporal Slices Gradient Magnitude Similarity Deviation. 16, $17,19,25,42,45-48,50,52,55,63$

STRRED Spatial-Temporal Reduced Reference Entropy Difference. 16, 17, 20, 22, 45-48, 50, $52,54,56,63$

STS Spatial-Temporal Slices. 16, 19, 20

SVM Support Vector Machine. 38, 53

TRRED Temporal Reduced Reference Entropic Differences. 21, 22

ViS3 Video Quality Assessment via Analysis of Spatial and Spatial-Temporal Slices. 16, 17, 23, $25,45-48,50,52,54,56,63$

VQEG Video Quality Experts Group. 41 


\section{INTRODUCTION}

In recent years there has been an increasing demand for video-based services. According to Cisco in 2019, 80 percent of all consumer Internet traffic will be from video applications [5]. As consumption increases, the demand for a better user experience also increases. Many factors contribute to the quality of the user experience, like the display in which the video is playing, the ambient light, the user interest, and the video quality. It is worth pointing out that video quality refers to the quality of the video signal, as perceived by the user.

There are basically two ways of assessing the quality of a video: subjectively and objectively. Subjective video quality assessment methods estimate the quality of a video by performing a series of pycho-physical experiments. In these experiments, subjects watch a series of videos and give a quality score to each video. The average of these scores is the Mean Opinion Scores (MOS), which is a subjective quality estimate. This type of video quality assessment method is known to be the most reliable method [6]. However, psycho-physical experiments are expensive, very difficult to replicate, and require a minimum number of subjects taken from a diverse pool of people to provide reliable results. Most of the times, objective video quality assessment methodologies are used. Objective methods are basically algorithms that automatically compute a quality estimate score for the video. These methods are faster and cheaper than the subjective methods, but they are less precise.

In the early days, image quality assessment methods were adapted to measure the video quality. In other words, image quality assessment methods, like Peak Signal-to-Noise Ratio (PSNR), were computed for each frame and averaged to provide the video quality predicted score [7]. However, using image quality assessment methods has limitations. The main issue is that these methods do not account for temporal distortions. To solve this problem, some research add temporal information to image quality assessment methods to improve their prediction accuracy performances. For example, Wang et al. proposed adding a motion estimation stage to the Structural Similarity Index (SSIM) [1], giving more weight to slow moving regions [8]. Vu et al. adapt the Most-Apparent-Distortion (MAD), an image quality assessment method, to video by using spatial-temporal slices and optical-flow [9].

Nowadays, most of the proposed video quality assessment methods use spatial and temporal features to evaluate video quality. For example, Pinson et al. extracted several features from the distorted and reference video to estimate the video quality [10]. Some of the features extracted by this method include spatial activity, temporal activity, and color-based differences. Seshadrinathan used a 3D Gabor analysis to extract spatial, temporal, and spatial-temporal features from the distorted video and compared them with the same features extracted from the reference (unimpaired) video [11].

Although video quality assessment methods are becoming more precise, there are still chal- 
lenges in this area. Chandler and Wang discuss some of these challenges related to image/video quality assessment methods [12,13]:

- How to deal with multiple distortions: Many video quality assessment methods have a very good performance when the content evaluated has the same distortion, i.e. when only the level of the distortion is modified from one video to another. However, different video distortions affect the video in different ways and some video quality methods cannot interpret which distortion is more or less visible.

- How to deal with image enhancements: Some video/image post-processing can improve the perceptual quality of the video or image. Nevertheless, most video quality assessment methods interpret this processing as a distortion, i.e. are not able to recognize quality enhancements.

- How to make the image/video quality assessment scores easy-to-use and easy-to-understand: Image and video quality assessment methods are becoming more complex over the years. Although most methods have a good correlation with subjective scores, they are often complex algorithms that are hard to understand and use.

- How to estimates video quality of High Dynamic Range (HDR) content: HDR type content is becoming more common, but most video quality assessment methods are not ready to evaluate the quality of this type of content.

Finally, one big challenge that is mentioned in both articles:

- How to reduce the runtime performance of video quality assessment methods: Some of the current video quality methods are so computationally complex that it is impossible to use them in real time scenarios or any practical application. For example, the average runtime of a recent developed method, the ViS3, is more than 600 seconds for videos 10 seconds long [12].

Some video quality methods have options to reduce the runtime. For example, Wang's video quality method has an option to reduce the number of frames used by the algorithm to estimate the video quality [8]. The VQM reduces the spatial and temporal resolution of the video to improve the runtime performance [10]. And MOVIE analyzes the video in an 8 -by- 8 frame interval [11]. But, although these methods offer options to improve the runtime performance, they do not consider that different video distortions are more or less sensitive to reductions of spatial or temporal resolution.

Our main objective is to create a framework to improve the runtime of video quality assessment methods, without affecting their prediction accuracy performance. One of the simplest methods to improve runtime performance is to reduce the video spatial resolution. However, when the spatial resolution is reduced, the video quality may be altered and the accuracy performance of the video quality assessment methods may decrease. In this work, we test the proposed 
framework using six video quality assessment methods. More specifically, we analyze their accuracy performance when we reduce the video spatial resolution. We also try to understand which video distortions are more (or less) sensitive to spatial reductions. The proposed framework has four steps. The first step, classification, consists of identifying the video distortions that are more sensitive to a spatial resolution reduction. The second step, reduction, performs a resolution reduction, according to its distortion. The third step, quality prediction, uses an objective quality assessment method to measure the video quality. Finally, the fourth step adjusts the predicted score, making sure that the same scale is used for scores corresponding to videos with different resolutions.

In this work, we design two video distortion classifiers to perform the first step of the proposed framework. These two classifiers aim to identify which distortions are present in the video, so that the video spatial resolution can be adjusted according to the sensitivity of the distortion. We test the proposed framework with six objective quality assessment methods and on four different video quality database. We also perform tests to analyze the effects of reducing the temporal resolution on the video quality assessment methods accuracy performance. Notice that reducing the temporal resolution is another simple and fast way to decrease the runtime of quality assessment methods.

We divide this work into six chapters. In Chapter 2, we give details of a digital video system, briefly detailing video compression, processing, and transmission algorithms, along with common video distortions. In Chapter 3, we describe the video quality assessment methods and the video quality databases used in this work. In Chapter 4, we detail the proposed framework and video classifiers. In Chapter 5, we present the results of this work. Finally, in Chapter 6, we present our conclusion and discuss possible future works. 


\section{DIGITAL VIDEO SYSTEM}

Before a video reaches the final user, it has to go through some processing stages. Figure 2.1 shows an overview of a common digital video transmission system. First, the original video is compressed to reduce its size and, consequently, increase the transmission speed. The encoding stage includes the compression and the channel encoding. The result of the video compression stage is a bitstream, in which the coded data is divided into packets for transmission. These packets are encoded according to the transmission protocol requirements. Then, the video is transmitted through a channel that can be dynamic or static, packet-switched or circuit-switched. When the video data reaches the receiver, it is decoded and decompressed in the decoding stage [14].

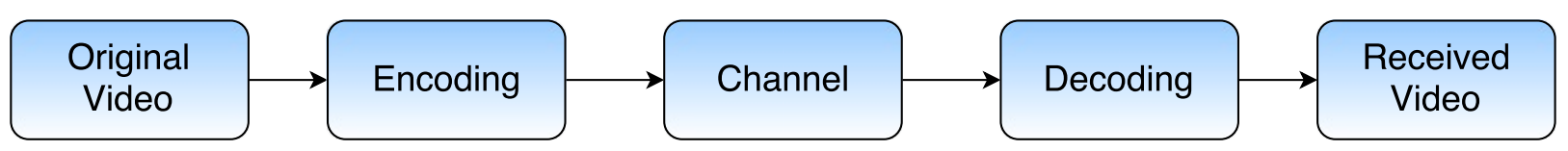

Figure 2.1: Overview of Video Transmission Diagram.

Video distortions are perceptible differences between the degraded video and the corresponding reference video. These distortions are the result of one or more errors introduced in the videos during compression, post processing or transmission stages. For example, the compression of a video, usually, is a lossy compression, meaning that some of the video data is discarded and the received video cannot be perfectly reconstructed [15]. Depending on the type of compression and the compression rate, some visible artifacts can be present in the video. Also, during the transmission stage through the physical channel, some of its packets can be lost, what may also affect the video quality. Even after the video is received and decompressed, it can further be processed for a better visualization on the display device. For example, if the video is in a lower resolution than the display resolution, the video can be upscaled to the display resolution, what may also alter its quality. Finally, the video can be corrupted by noise during the acquisition, transmission, storage, or post-processing stages [16].

Next, we briefly describe the several stages of a modern video transmission scenario and the several types of distortions that can be introduced in each stage.

\subsection{VIDEO COMPRESSION ALGORITHMS}

Data compression techniques have the goal of representing the data in a more compact form [15]. Videos are compressed to reduce storage and transmission costs. There are lossless and lossy compression algorithms. Although lossless compression algorithm can reduce the video size and recover it without any errors, the compression ratio that can be achieved with lossless compression 
algorithm is limited [17]. Fortunally, since video sequences are usually very redundant, lossy compression algorithms can achieve great compression rates with a good video quality. The most popular lossy compression algorithms are MJPEG, MPEG-2, DIRAC-Wavelet, H.264 and HEVC. Although lossy compression algorithms are more efficient in terms of compression rate, they may introduce perceivable artifacts into the video.

The MJPEG compression algorithm is based on the JPEG image compression. JPEG makes use of the Discrete Cosine Transform (DCT). First, the image is divided into 8x8 blocks and the DCT of each block is computed. The DCT coefficients are quantized, removing most of the high frequency coefficients. The quantized coefficients are rearranged and coded using a Huffman coding algorithm [18]. In MJPEG each video frame is compressed as a JPEG image. Although JPEG is very good at compressing images, using it to compress individuals video frames is not a very good strategy. Video sequences have temporal redundancies that can be explored to allows for a more efficient compression of video sequences. So, videos compressed by MJPEG have a lower video quality than videos compressed by other compression standards, like MPEG-2 and H.264, which take into account temporal redundancies.

Figure 2.2 shows an example of video frame compressed by MJPEG, which is affected by blocking and blurring artifacts. The blocking artifact is a consequence of the quantization of the DCT coefficients. Since the coefficients in each block are processed independently, regardless of the spatial correlation between them, intensity differences between neighboring blocks cause the edges/borders that characterize blockiness. The Human Visual System (HVS) can easily detect blocking artifacts because of equidistant distribution of the blocks in the frame [19]. Blurring artifacts, on the other hand are caused by any type of operation that discards high frequencies DCT coefficients [19]. In image processing, the high frequencies coefficients are associated with edges and abrupt changes in intensities [20]. Therefore, blurred images have less sharp edges and borders.

MPEG-2 compression algorithm is a block-based motion-compensated video coding algorithm. It is similiar to JPEG, but it uses motion estimation techniques to reduce temporal redundancy. Since it also individually quantizes the DCT blocks, discarding most of the high frequencies components, MPEG-2 may also introduce blockiness and blurring artifacts in the compressed video. Motion estimation techniques search blocks in previous frames that are similar to blocks in the current frame [21]. This way, only the coordinates of the similar blocks and the difference between these blocks and the current blocks need to be encoded and sent to the receiver. As a consequence of the motion estimation, videos compressed by MPEG-2 may also be affected by mosquito noise. This artifact occurs when the predicted block contains only part of a moving object. Mosquito noise is more visible in smooth texture regions around egdes [14]. Figure 2.3 shows a video frame compressed by MPEG-2 and the corresponding reference frame.

H.264 compression algorithm is also a block-based motion-compensated video coding algorithm [22]. In H.264, the video is first divided into macroblocks $(16 \times 16$ for the luminance component and $8 \times 8$ for the chroma components), and, then, the DCT coefficients of each macroblock 


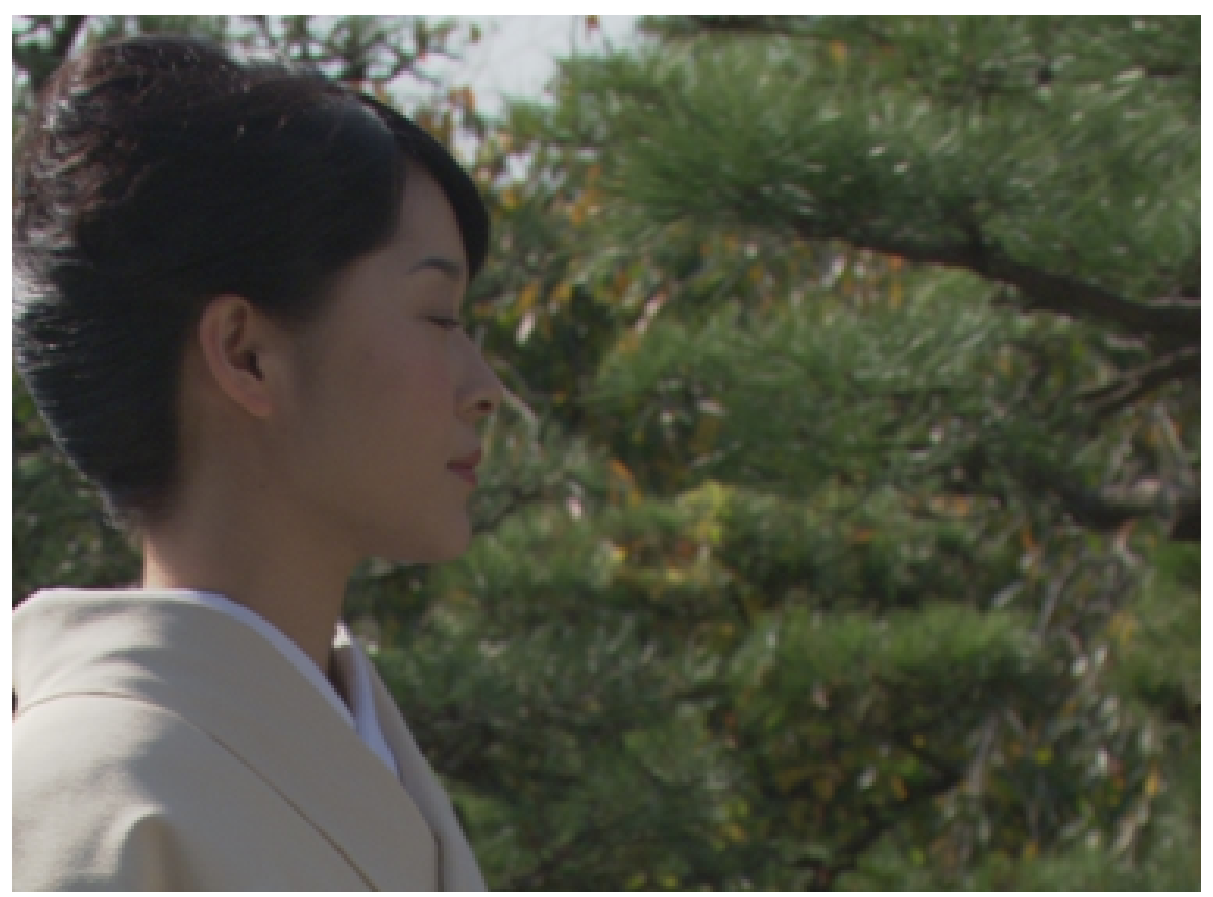

(a) Reference Frame

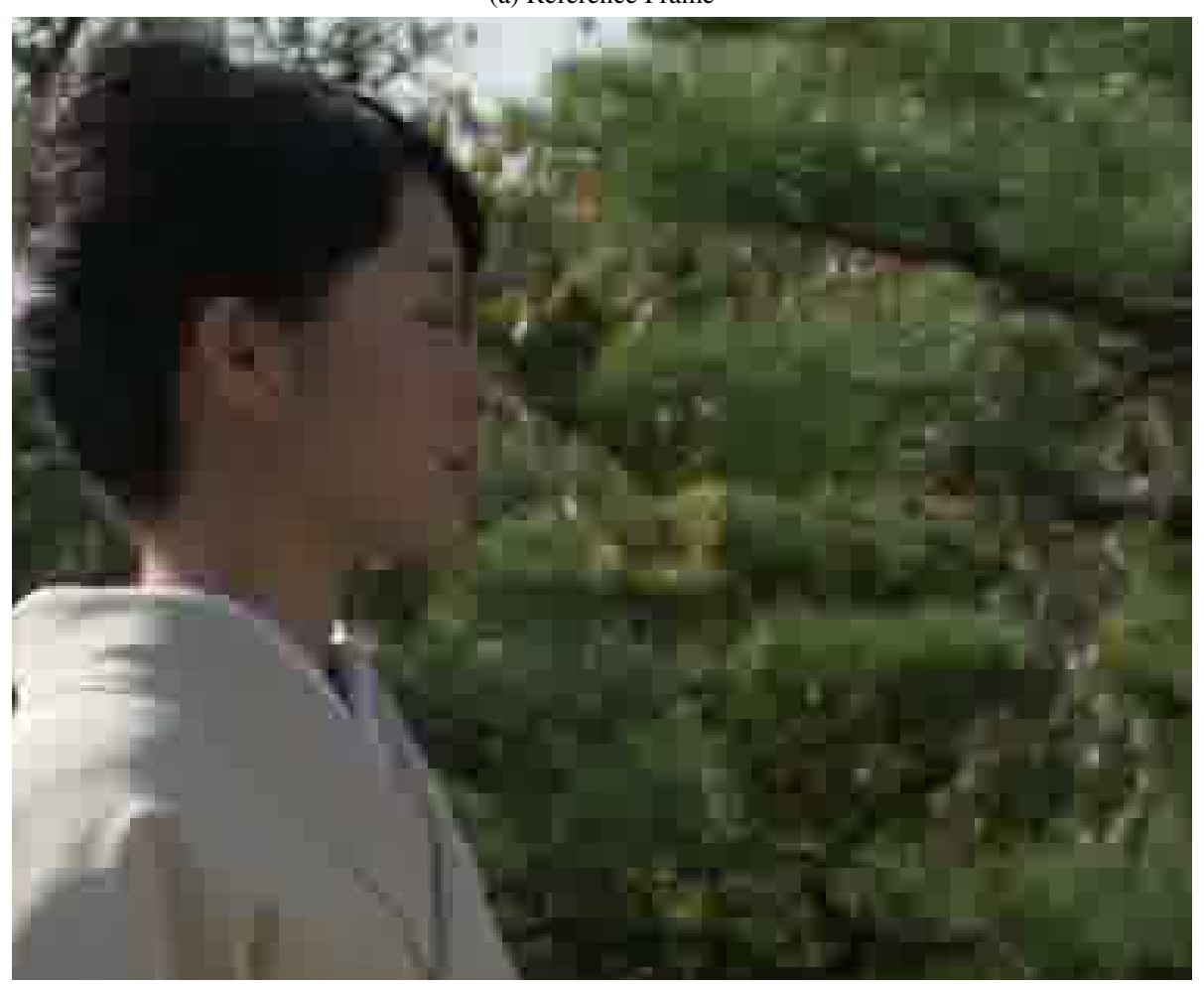

(b) MJPEG

Figure 2.2: Comparison between (a) the reference frame and (b) the frame compressed using a MJPEG algorithm.

are calculated and quantized. Next, a motion estimation algorithm is used to obtain inter-frame predictions. Different from previous compression algorithms, each macroblock can be divided into sub-macroblocks of different sizes, raging from $16 \times 16$ down to $4 \times 4$. This granularity improves the quality of the prediction, specially for fast motion regions. Koh et al. show that the 


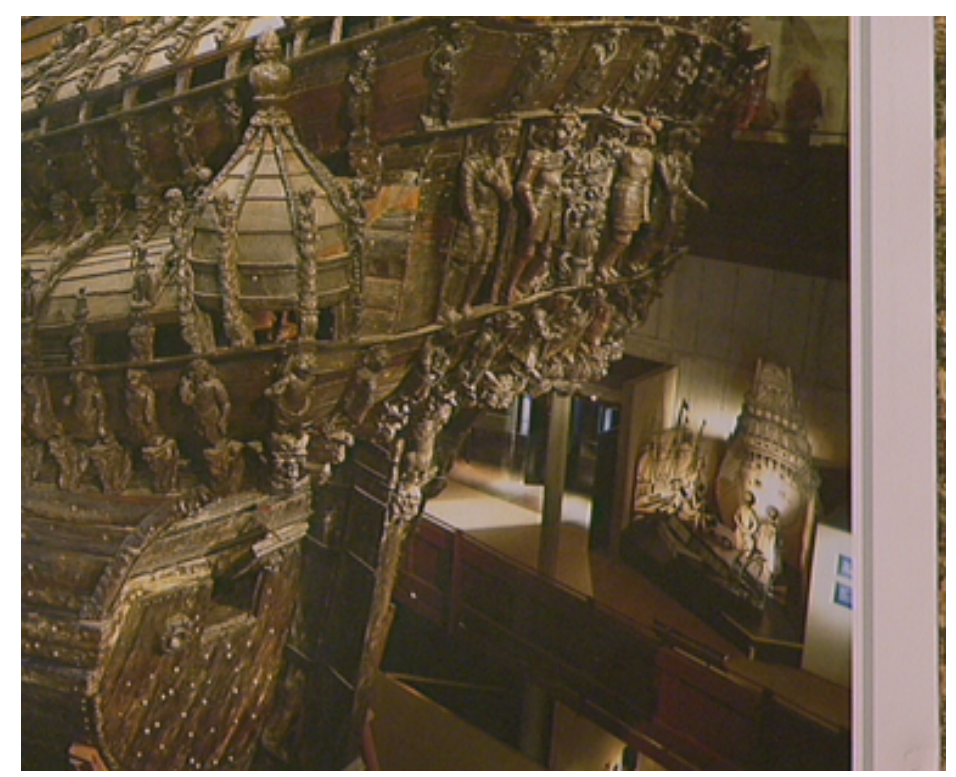

(a) Reference Frame

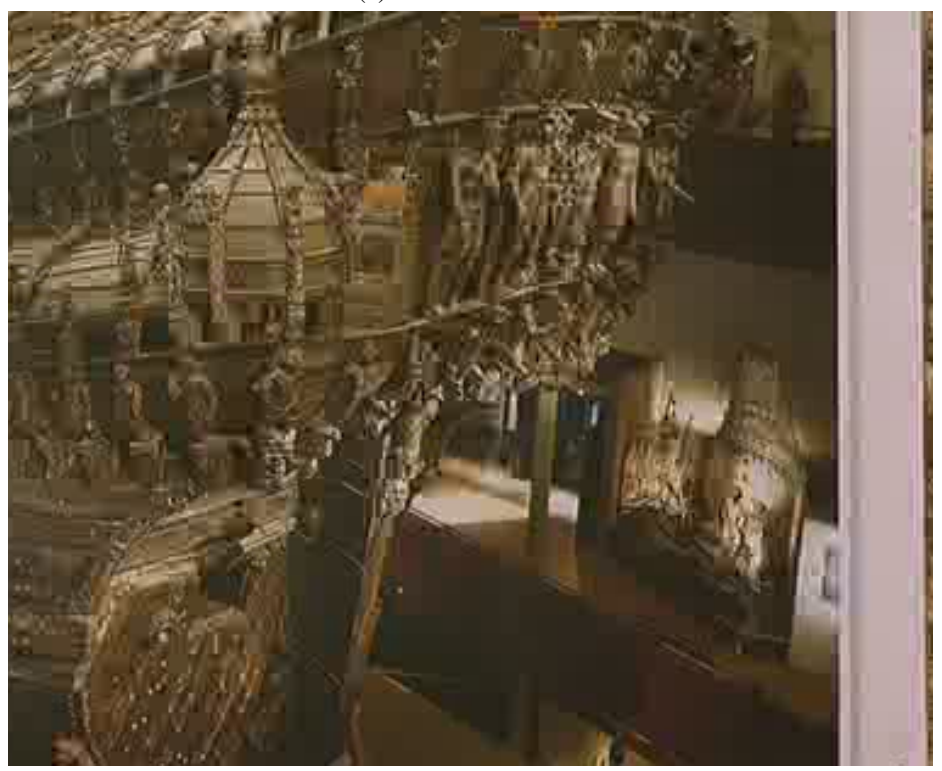

(b) MPEG-2

Figure 2.3: Comparison between (a) the reference frame and (b) the frame compressed using a MPEG-2 compression algorithm.

blocking is the most annoying artifact present in videos compressed by MPEG-2 [23]. To improve video quality, H.264 uses a deblocking filter to reduce the blocking artifacts, which are common in other block-based compression algorithms. The use of a deblocking filter improves the quality of the video by $9 \%$ in PSNR [24]. Figure 2.4 shows a H.264 compressed video frame and the corresponding reference frame. Notice that blocking artifacts are less visible in H.264 compressed frames than in MJPEG or MPEG-2 compressed frames. However, H.264 compressed video frames still present blurring artifacts.

DIRAC-Wavelet compression algorithm was developed by the British Brodcasting Corporation (BBC) [25]. The major difference between the DIRAC-Wavelet algorithm and other com- 


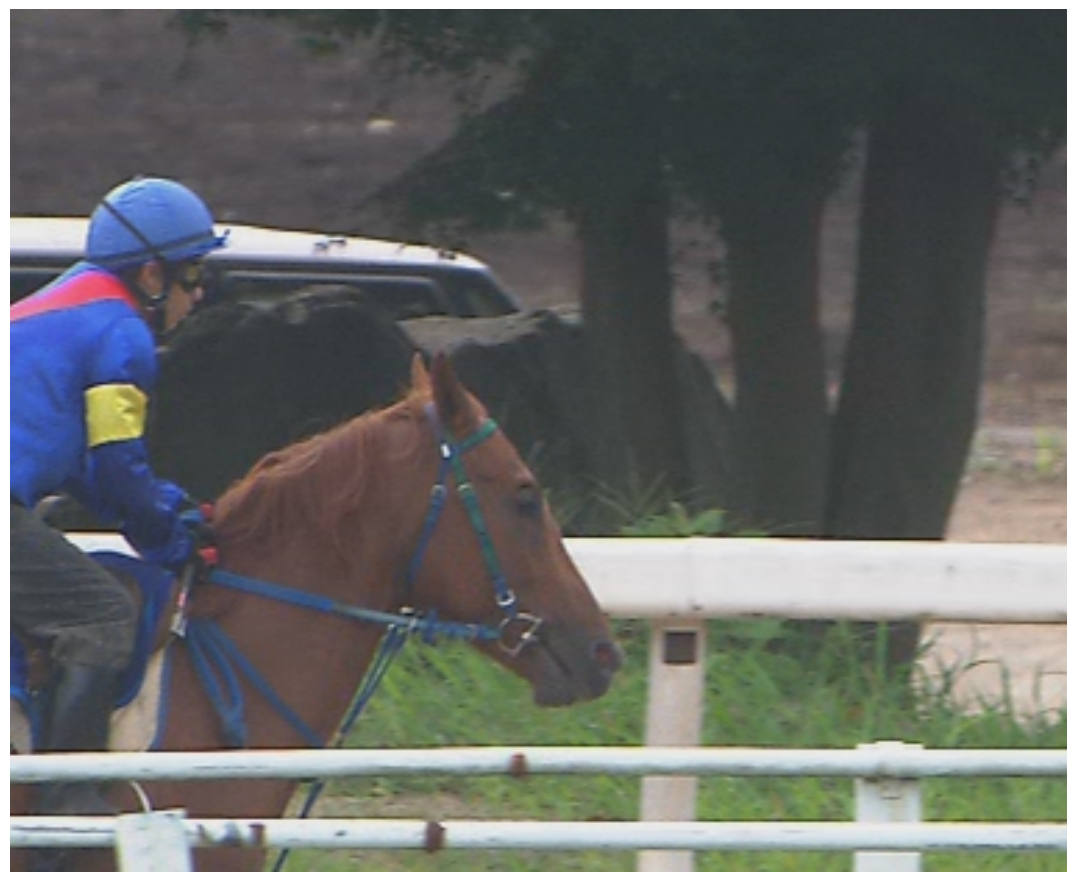

(a) Reference Frame

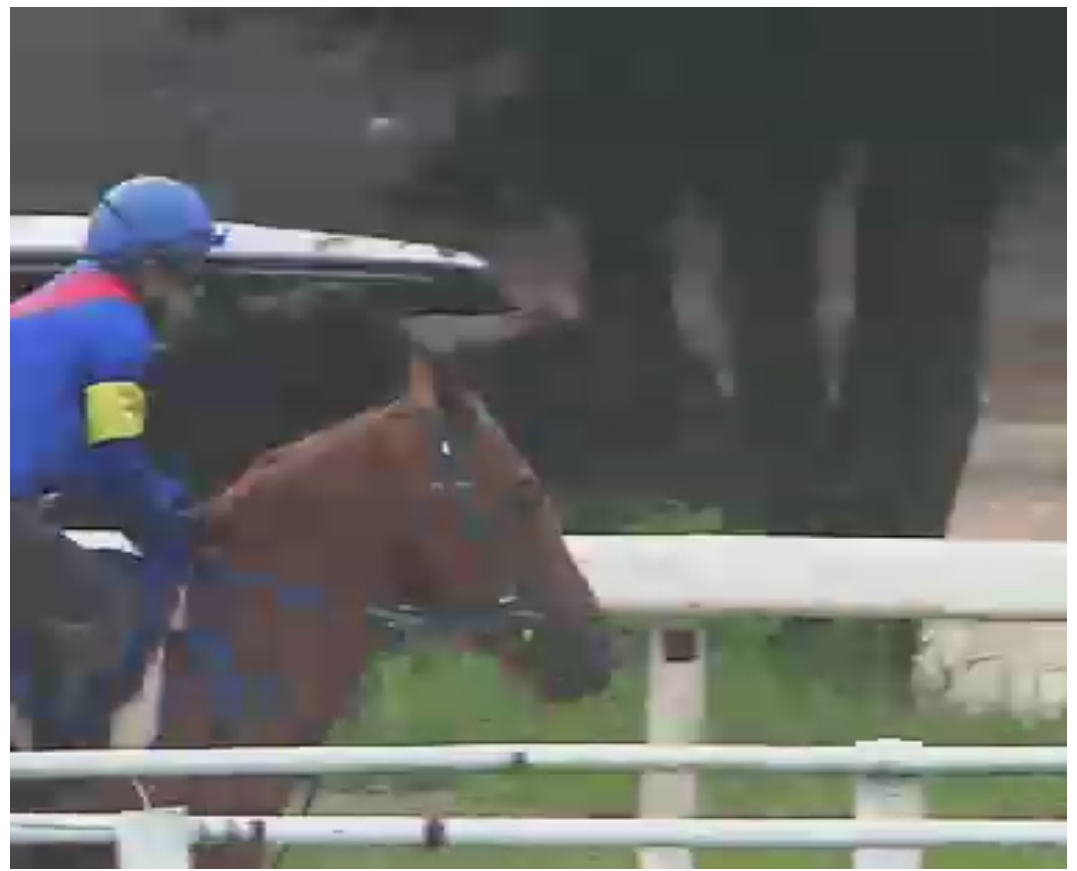

(b) H.264

Figure 2.4: Comparison between (a) the reference frame and (b) the frame compressed using an H.264 compression algorithm.

pression algorithms, like MPEG-2 and H.264, is that it uses wavelets instead of DCT, reducing the blockiness artifacts. But, the quantization of wavelet coefficients leads to ringing and blurring artifacts. Ringing artifacts manifest themself as wave-like structures in edges regions. Figures 2.5 shows an example of a video frame distorted by a DIRAC-Wavelet compression. Notice that ringing artifacts are present in areas around high contrast edges. 
H.265 or HEVC compression algorithm is an improved version of the H.264 compression. One of the changes introduced by H.265 is the possibility of partitioning the video frame using blocks of different sizes, which vary from $64 \times 64$ down to $8 \times 8$ [26]. The performance of H.265 is $50 \%$ better than the performance of H.264, i.e. for the same quality level, the bitrate obtained with H.265 is half the bitrate obtained with H.264 [27]. Figure 2.6 shows a video frame distorted by a H.265 compression compression. Videos compressed with H.265 at low bitrates contain mostly blurring artifacts.

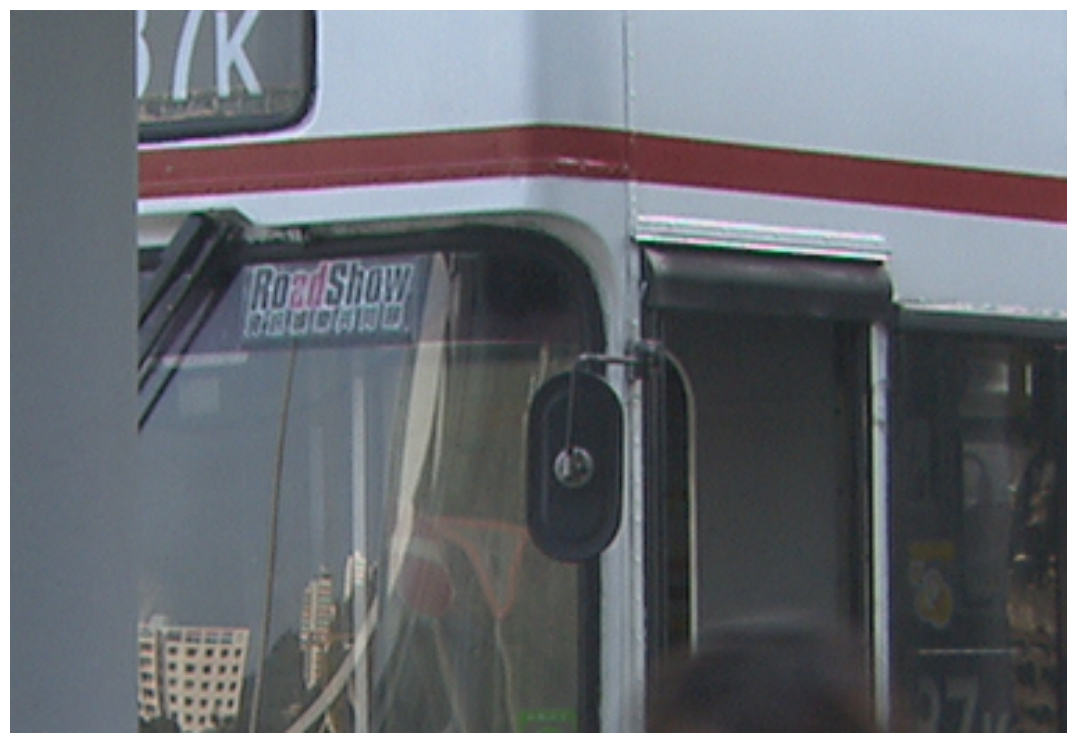

(a) Reference Frame

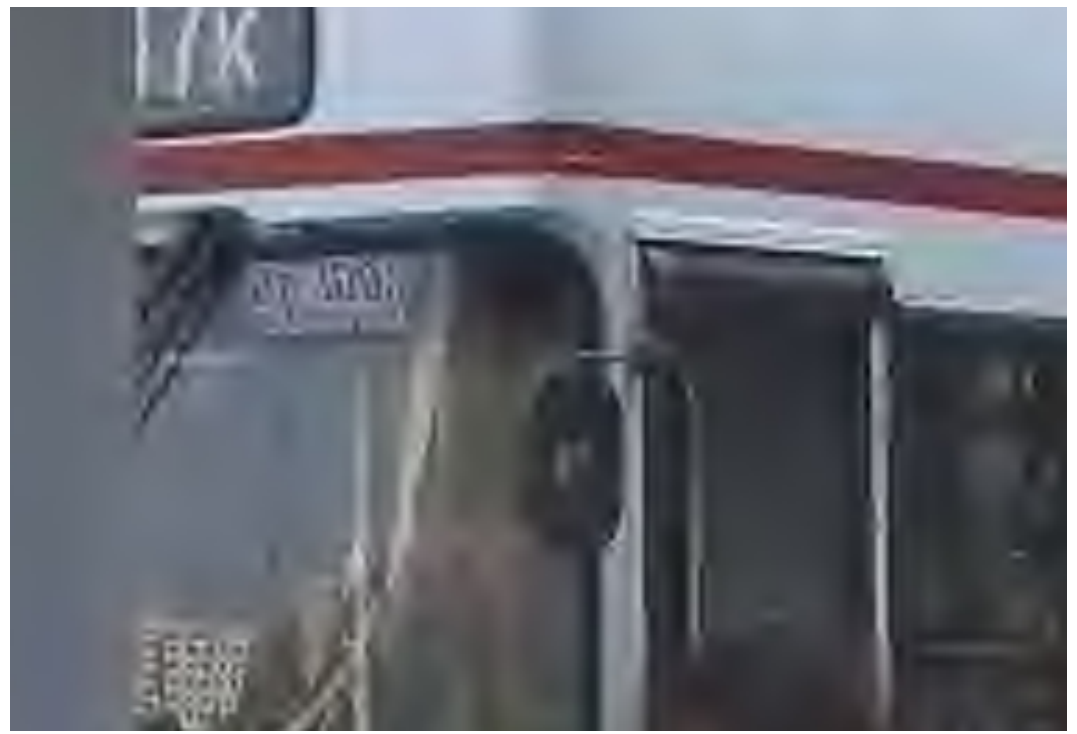

(b) DIRAC-Wavelet

Figure 2.5: Comparison between (a) the reference frame and (b) the frame compressed using a DIRAC-Wavelet compression algorithm. 


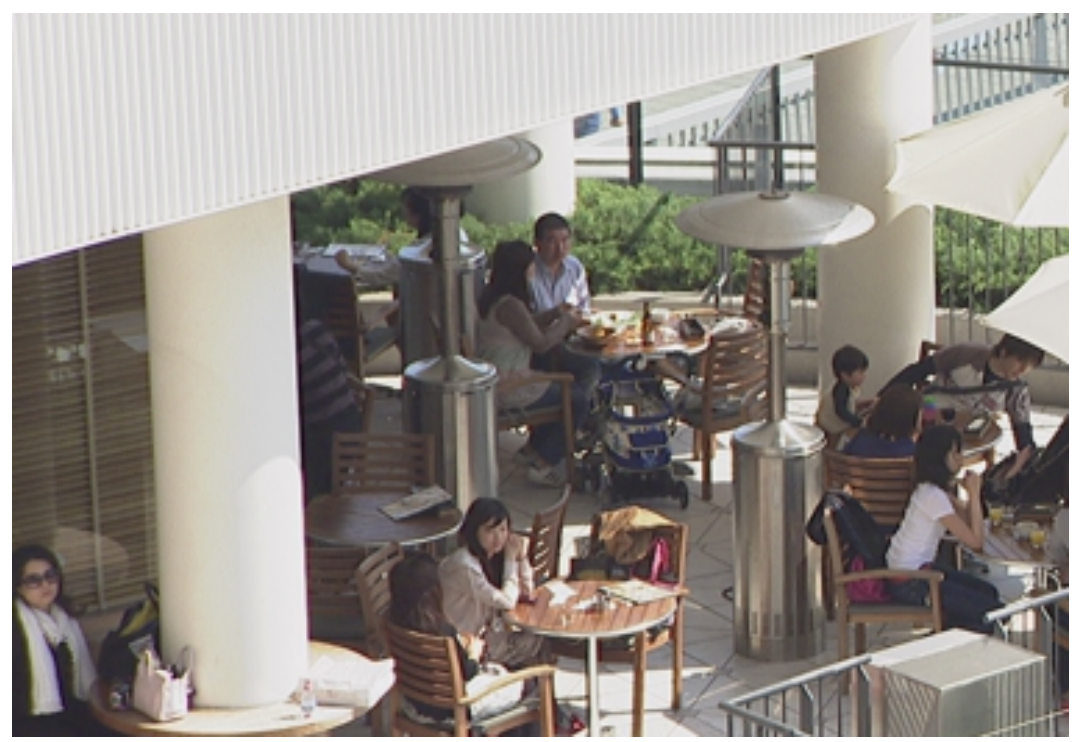

(a) Reference Frame

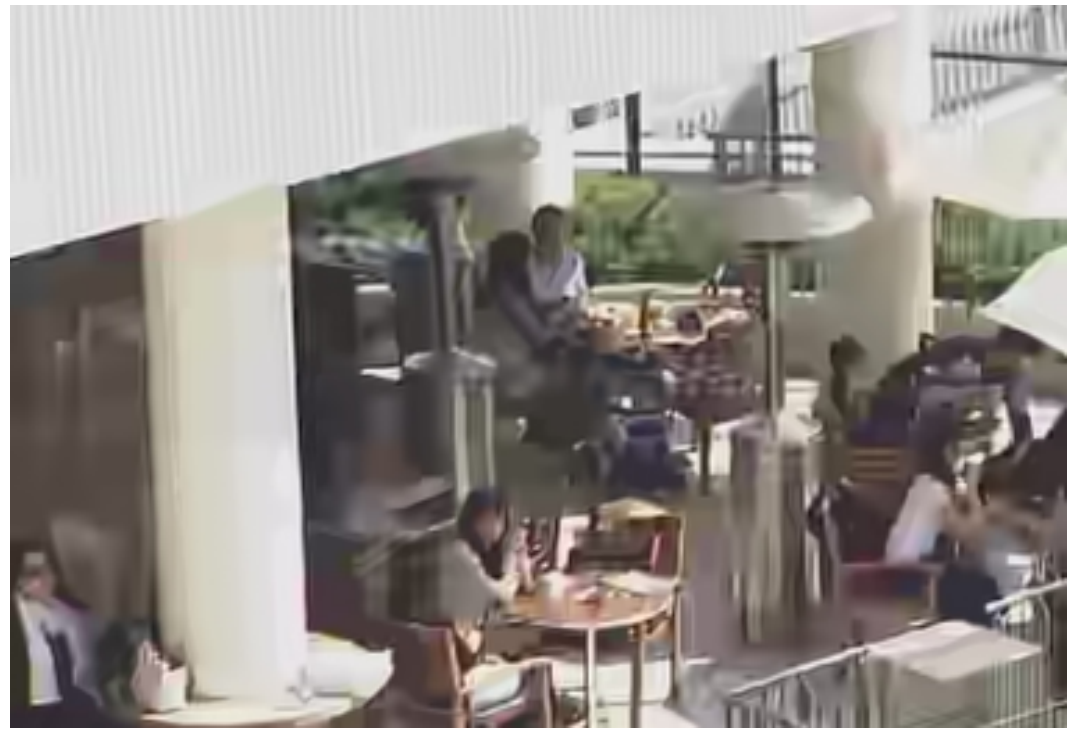

(b) HEVC

Figure 2.6: Comparison between (a) the reference frame and (b) the frame compressed using an H.265 compression algorithm.

\subsection{UPSCALING PROCESSING}

Sometimes the display where the video is presented has a higher resolution than the received video. Algorithms of interpolation are then used to upscale the video resolution to the display resolution. This type of processing is commonly used in streaming services applications, because video resolutions vary according to the available bandwidth [28]. Interpolation algorithms used in upscaling consider the neighboring pixels to estimate a pixel value.

There are different interpolation algorithms, including nearest-neighbor, bilinear, and bicubic [29]. The nearest-neighbor algorithm is the most simple interpolation algorithm, consisting simply of repeating the value of the neighboring pixel that is closer to the pixel. The biliniar 
and bicubic generate images with better quality, because they use more information to predict the new pixel. However, the images generated using these interpolation algorithms are blurred. Figure 2.7 shows an example of video frame distorted by upscaling. In this example, the video was compressed with H.264 and then upscaled to a higher resolution, using a bilinear interpolation algorithm. Notice that the upscaled video frame is more blurred than the reference video frame.

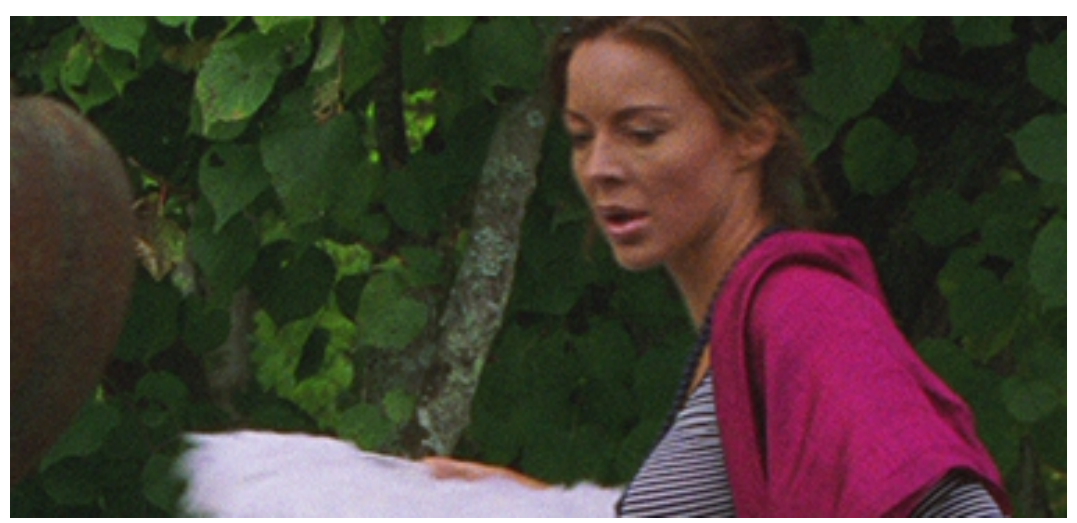

(a) Reference Frame

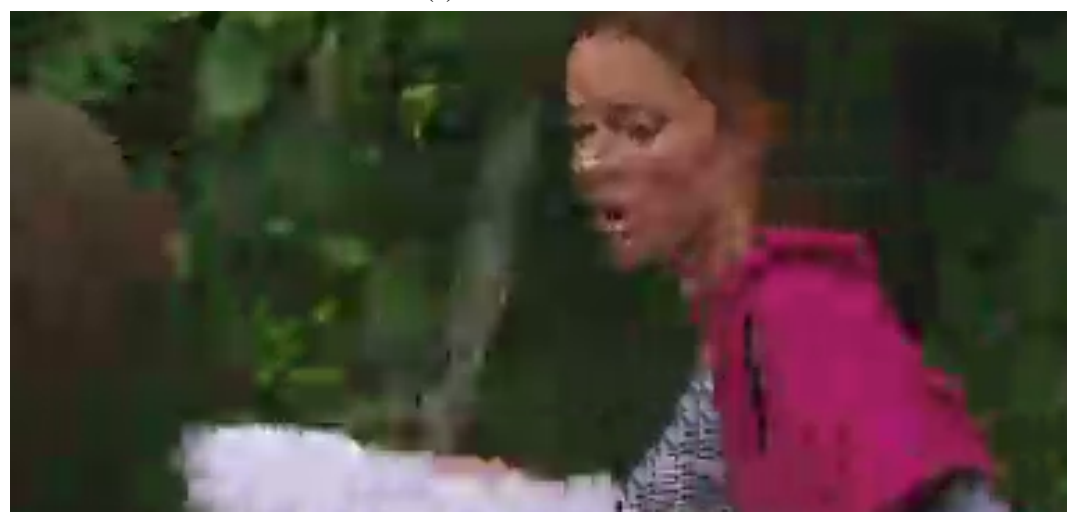

(b) H.264

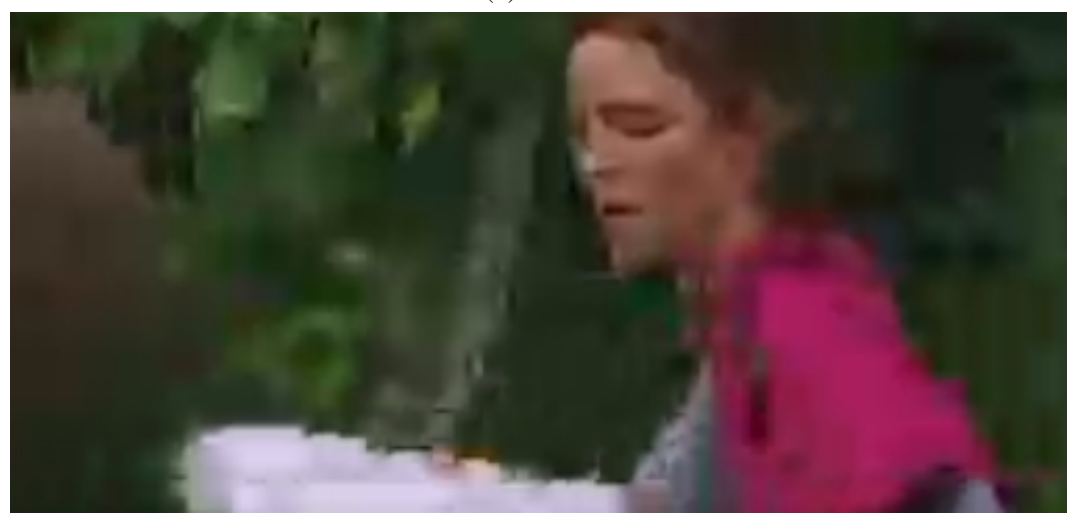

(c) H.264 with Upscaling

Figure 2.7: Comparison between (a) the reference frame and (b) the frame compressed by an H.264 algorithm and (c) the frame compressed by an H.264 algorithm with Upscaling. 


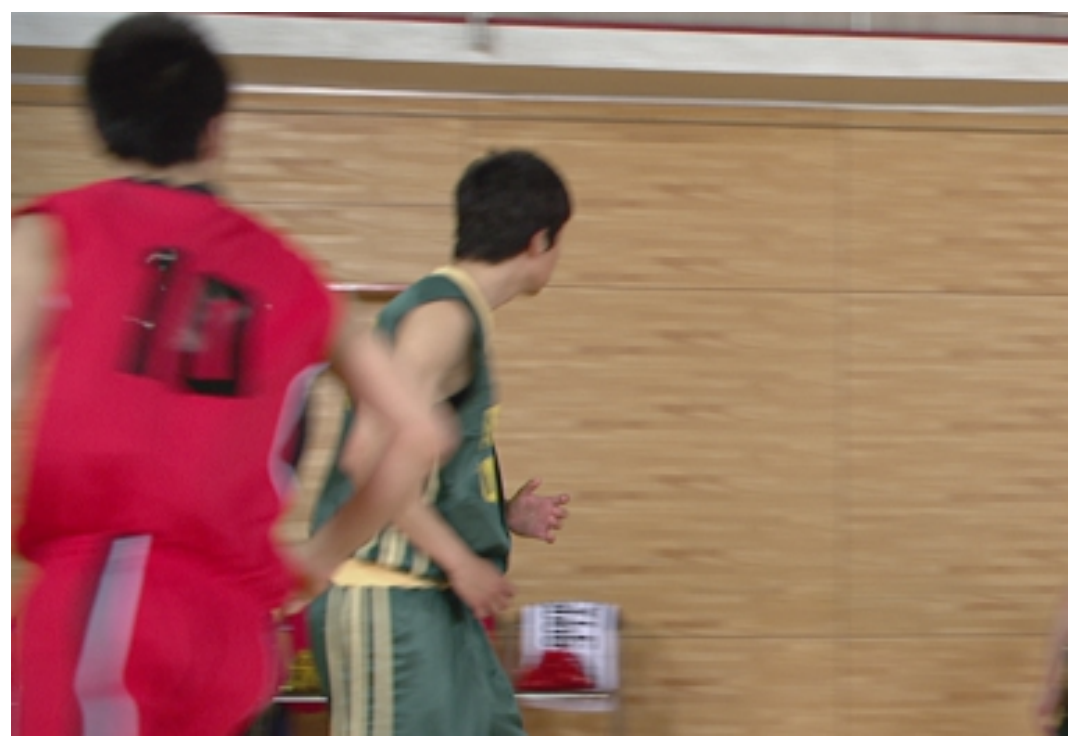

(a) Reference Frame

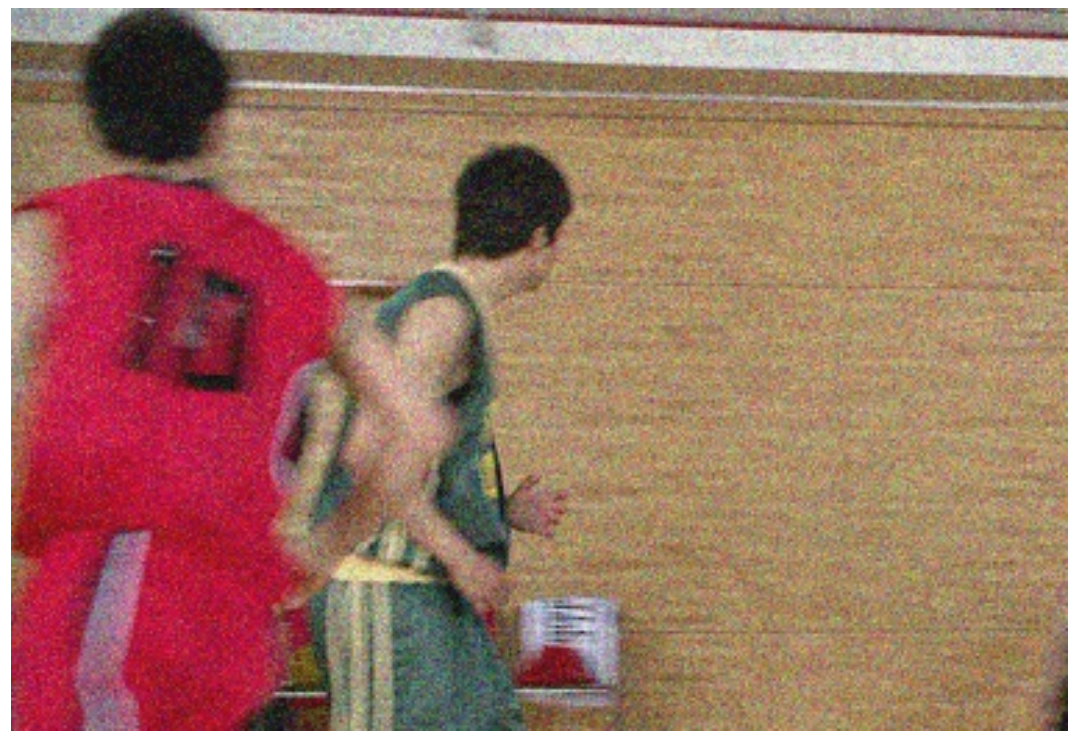

(b) White Noise

Figure 2.8: Comparison between (a) the reference frame and (b) the frame distorted by white noise.

\subsection{VIDEO TRANSMISSION}

Video can be transmitted either in analog or digital channels. In analog transmissions, one of the most common artifacts is noise, most commonly white noise that is a random signal that occupies the whole spectrum. Noise can also be added in video sequences during the acquisition and processing [16]. Figure 2.8 shows a video frame distorted by white noise. Notice that in this case the noise is an additive distortion, which affects the whole frame.

In digital transmission, before the compressed video is transmitted over a channel (e.g. a wired or wireless transmission channel), it is divided into packets. Sometimes these packets are discarded due to the traffic in the network. The impact of a packet loss in video quality depends on the content of the video and the channel loss distribution [30]. Packet loss is characterized 


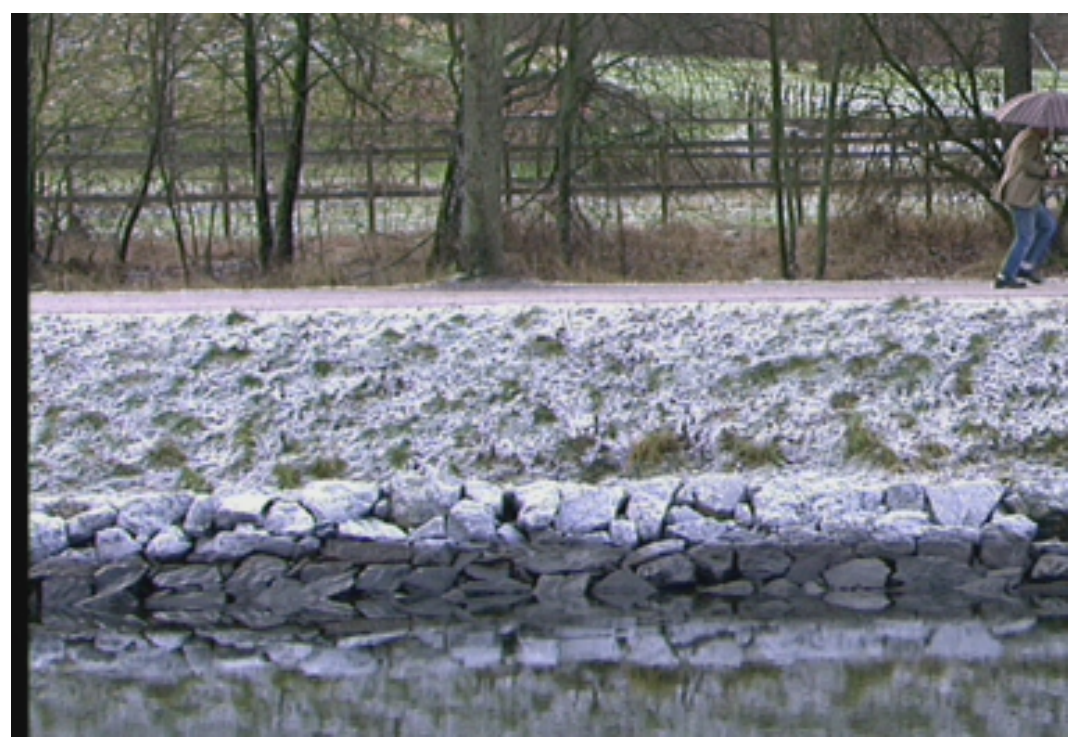

(a) Reference Frame

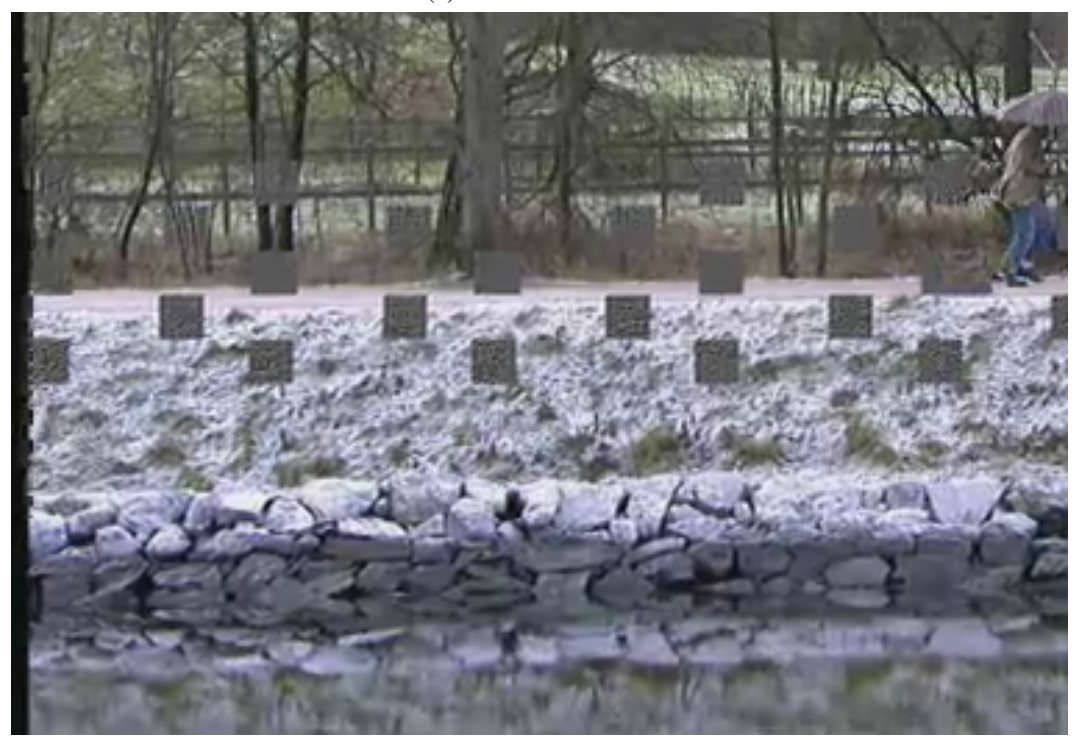

(b) Packet Loss

Figure 2.9: Comparison between (a) the reference frame and (b) the frame distorted by packet loss artifacts.

by the presence of erroneously decoded blocks in the decoded video. In most video compression algorithms, frames that are coded without any reference to previous frames are send periodically. These frames are called I-Frames and are used as a reference to decode other frames. If the packet loss occurs in these I-Frames, the distortions are more severe and may affect several frames [31]. Figure 2.9 shows a video frame distorted by packet-loss artifacts. Notice that packet-loss is a local distortion, i.e. it is spatially and temporally concentrated. 


\section{VIDEO QUALITY ASSESSMENT METHODS}

In this chapter, we discuss objective methods to automatically estimate the quality of visual signals (images and videos). More specifically, we present the different types of objective methods and present a review of the state-of-the-art of the area, describing the methods that are used in this work. Finally, we also present the video quality assessment databases that are used as benchmarks for the tests performed in this work.

\subsection{OBJECTIVE QUALITY ASSESSMENT METHODS}

There are two ways to evaluate the video quality. The first is to subjectively assess the quality of videos. Subjective quality assessment methods are psycho-physical experiments in which subjects (voluntary participants) evaluate the quality of a set of videos. To perform a subjective test properly, experimenters follow recommendations established by the International Telecommunication Union (ITU) [32]. Subjective methods are known to provide the most reliable results for video quality, however, they are very expensive and time-consuming.

The second way to evaluate the video quality consists of measuring the signal quality using objective methods. These methods use mathematical models, which can be implemented in hardware or software, to evaluate video quality. Depending on the information available, objective methods can be categorize into three groups:

- Full-Reference (FR): the original and the test video are available to the method at the measurement point;

- Reduced-Reference (RR): besides the test video, some information about the original video is available to the method at the measurement point;

- No-Reference (NR): only the test video is available to the method at the measurement point.

Since FR methods require information about the reference video, what is frequently not available in real-time applications, these methods are more adequate for laboratory applications. Codec comparison and optimization are examples of scenarios in which these methods can be used. Although FR methods are more precise, RR and NR methods are more appropriate for real-time scenarios.

Objective quality assessment methodologies can also be classified as data metrics or picture metrics [33]. Data metrics measure only the fidelity of the video signal, i.e. how similiar are the test and reference video. Data metrics do not consider the content of the video or how degradations are perceived by human viewers. These metrics, often, are simple and fast. Mean Squared Error (MSE) and Peak Signal-to-Noise Ratio (PSNR) are examples of data metrics. One of the 
problems of data metrics is that they do not consider the type of distortion present in the image or video. Figure 3.1 shows five images that have the same MSE score, but are degraded with different types of distortions and have different perceived quality levels. As can be seen in these images, although the perceived quality of the images containing the distortions are different, the MSE does not capture these differences. Another problem of data metrics is that they do not take into consideration the content of the image or video. According to Wang, distortions may be less visible in texture areas of the video frame or when the speed of motion is large [8]. Nevertheless, it is worth pointing out that data metrics can be used in other contexts, when we simply want to measure the differences between two video signals.

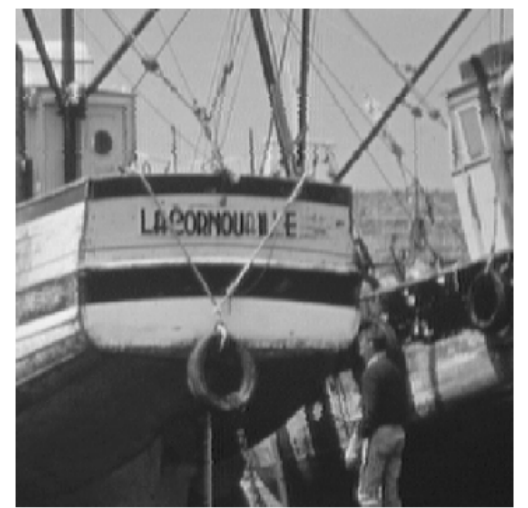

(a)

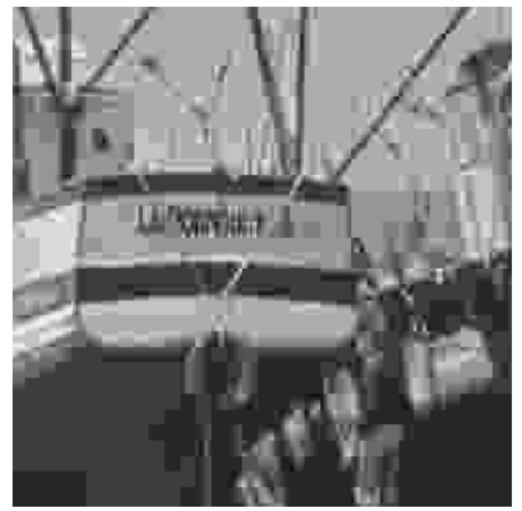

(d) $\mathrm{MSE}=210$ $\operatorname{SSIM}=0.6949$

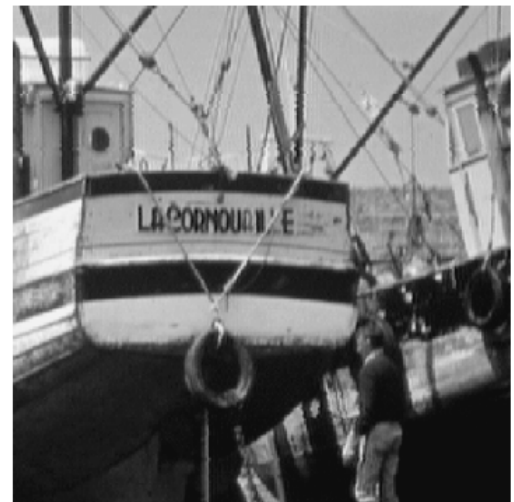

(b) $\mathrm{MSE}=210$

$\operatorname{SSIM}=0.9168$

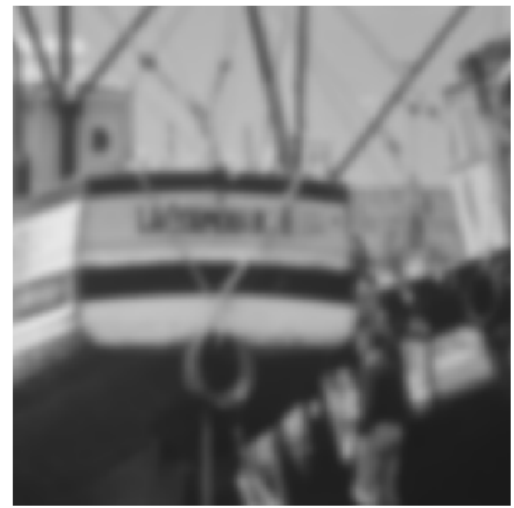

(e) $\mathrm{MSE}=210$

$\operatorname{SSIM}=0.7052$

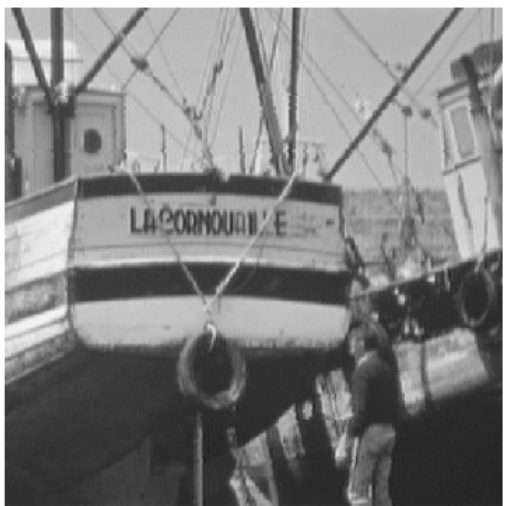

(c) $\mathrm{MSE}=210$ $\mathrm{SSIM}=0.9900$

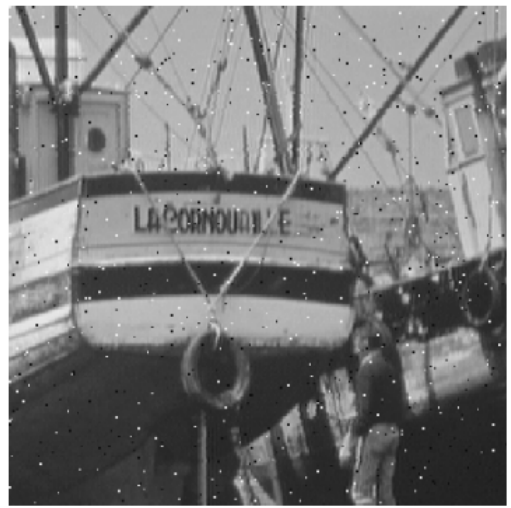

(f) $\mathrm{MSE}=210$ $\operatorname{SSIM}=0.7748$

Figure 3.1: Comparison between the images with the same MSE score, but with different distortions. (a) Original Image. (b) Contrast-stretched image. (c) Mean-shifted image. (d) JPEG compressed image. (e) Blurred image. (f) Salt-pepper impulsive noise contaminated image. Original From [1].

In the last decades, researchers started developing pictures metrics, which are objective quality assessment methods that take into consideration the intrinsic characteristics of a visual signal. In other words, picture metrics analyze the visual data as visual information, considering the effects of video distortions and content on the perceived quality. Picture metrics can be designed either using a vision modeling approach or an engineering approach. The vision modeling approach implements models of components of the HVS, like color perception, contrast sensitivity, and pattern masking. One example of a video quality assessment method that uses a vision modeling 
approach is the Sarnoff Just Noticeable Differences (JND) method, which is based on the chromatic and luminance differences that can be perceived by human viewers, i.e. the just noticeable perceived difference [34]. Another example of a video quality assessment method that uses visual models is the Perceptual Distortion Metric (PDM), which is implements a perceptual color space model, multi-channel representation of spatial and temporal mechanisms, contrast sensitivity and pattern masking models [35].

The engineering approach is based on the analysis of certain features of the video, like the spatial luminance gradient, the image structure, or specifics artifacts (like blur, noise, etc). Some examples of video quality assessment methods that adopt an engineering approach are:

- SSIM - an image quality assessment method, that measures the mean, variance and covariance of patches of the image [1];

- Gradient Magnitude Similarity Deviation (GMSD) - an image quality assessment method that analyzes the spatial luminance gradient [36];

- Spatial and Spatial-Temporal Slices Gradient Magnitude Similarity Deviation (SSTS-GMSD) - an extended version of the GMSD that analyzes the gradient of the Spatial-Temporal Slices (STS) of the video [2].

On the other hand, there are methods that combine both approaches. One example is the Video Quality Assessment via Analysis of Spatial and Spatial-Temporal Slices (ViS3), which includes a data metric and a picture metric in its model [4]. First, a spatial distortion map is computed using the Most Apparent Distortion algorithm (MAD), which takes into account on how the HVS perceives video distortions [37]. Then, the spatial-temporal dissimilarity between the reference and distorted videos is computed using spatial-temporal correlation and spatialtemporal responses.

Recently, the use of machine learning algorithms for video quality assessment has become extremely popular, specially for the no-reference quality assessment methods scenario. Most machine learning based quality assessment methods extract features from the videos and use machine learning techniques, like support vector regression or neural networks, to predict the video quality scores. Xu et al. propose a no-reference video quality assessment method that uses an unsupervised feature learning approach that trains a support vector regression to predict video quality [38]. Saad et al. use a spatio-temporal natural scene statistics model and a motion model to train a support vector regression algorithm [39].

Although prediction accuracy performance of video quality assessment methods are improving, these methods are becoming more complex, requiring large running times. Both ViS3 and Spatial-Temporal Reduced Reference Entropy Difference (STRRED) take several minutes to estimate the quality of a video 10 seconds long [4, 40]. MOVIE, an algorithm that uses a 3D Gabor filters, takes hours to estimate the quality of a video 10 seconds long [11]. Therefore, an mentioned earlier, improving the runtime of video quality assessments methods is still an open challenge $[12,13]$. 


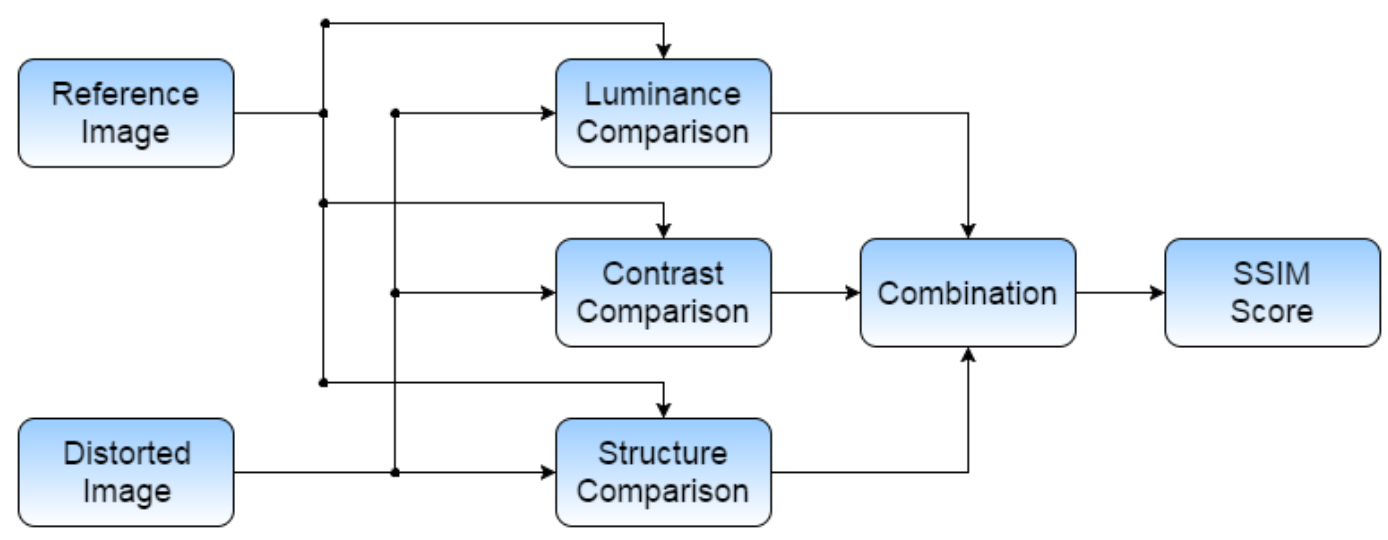

Figure 3.2: Diagram of the SSIM Algorithm.

In this work, we study the performance of full-reference and reduced-reference video quality assessment methods. Among the assessment methods studied in this work are the ViS3, SSTSGMSD, GMSD, SSIM and PSNR FR methods and the STRRED RR method. These methods are detailed in the next sub-sections.

\subsubsection{PSNR}

The Peak Signal-to-Noise Ratio (PSNR) is a simple full-reference method. PSNR is based on the mean squared error (MSE) difference of two images, given by the following equation:

$$
M S E=\frac{\sum_{x=0}^{M-1} \sum_{y=0}^{N-1}\left(I_{r e f}(x, y)-I_{\text {dist }}(x, y)\right)^{2}}{M \cdot N},
$$

where $I_{r e f}(x, y)$ is a pixel from the reference image, $I_{\text {dist }}(x, y)$ is a pixel from the distorted or test image and $M \mathrm{x} N$ is the spatial dimension of these images. In this work, we consider only the luminance component of the image to calculate the MSE.

PSNR is calculated using the following equation:

$$
P S N R=10 \cdot \log _{10} \frac{M A X_{I}^{2}}{M S E},
$$

where $M A X_{I}$ is the maximum possible value of the image (for images with 8 bits, $M A X_{I}=$ 255). To use PSNR as a video quality method, we calculate the PSNR for each frame of the video and average the frame results to obtain one video quality score.

\subsubsection{SSIM}

The Structural Similarity (SSIM) is a full reference image quality assessment method developed by Wang et al. [1]. SSIM compares three features of the reference and distorted image. These features are luminance, contrast and structure, as shown in Figure 3.2. 
SSIM performs its analysis on the luminance component of the image. To compute the SSIM score, the image is first divided in $8 \times 8$ blocks. For each block the luminance, contrast and structure comparison measurements are performed. The luminance comparison is calculated by the following equation:

$$
l(x, y)=\frac{2 \mu_{x} \mu_{y}+C}{\mu_{x}^{2}+\mu_{y}^{2}+C},
$$

where $\mu_{x}$ and $\mu_{y}$ are the mean intensity of the original image block and the distorted (test) image block, respectively, and $C$ is a small constant necessary to avoid instability.

The Contrast Comparison is calculated by the following equation:

$$
c(x, y)=\frac{2 \sigma_{x} \sigma_{y}+C_{2}}{\sigma_{x}^{2}+\sigma_{y}^{2}+C_{2}}
$$

where $\sigma_{x}$ and $\sigma_{y}$ are the standard deviation intensity of the original and distorted (test) image block, respectively, and $C_{2}$ is a small constant necessary to avoid instability.

The Structural Comparison is calculated by the following equation:

$$
s(x, y)=\frac{\sigma_{x y}+C_{3}}{\sigma_{x} \sigma_{y}+C_{3}}
$$

where $\sigma_{x}$ and $\sigma_{y}$ are the standard deviation intensity of the original and distorted block, $\sigma_{x y}$ is the covariance between the original image block and the distorted (test) image block, and $C_{3}$ is a small constant necessary to avoid instability.

To combine these comparisons into a single SSIM map, we use the following equation:

$$
\operatorname{SSIM}(x, y)=l(x, y)^{\alpha} \cdot c(x, y)^{\beta} \cdot l(x, y)^{\gamma},
$$

where, to simplify, $\alpha=\beta=\gamma=1$. The average value of the SSIM map is the final score. To use SSIM as a video quality method, we calculate the SSIM for each video frame and average these values to obtain the video quality score.

\subsubsection{GMSD}

The Gradient Magnitude Similarity Deviation (GMSD) is a full reference image quality assessment method developed by Xue et al. [36]. It is based on the gradient differences between reference and distorted (test) gray-scaled images. The gradient magnitude is computed using Prewitt filters for horizontal and vertical directions, as given by the following expressions:

$$
h_{x}=\left|\begin{array}{ccc}
1 / 3 & 0 & -1 / 3 \\
1 / 3 & 0 & -1 / 3 \\
1 / 3 & 0 & -1 / 3
\end{array}\right|
$$

$$
h_{y}=\left|\begin{array}{ccc}
1 / 3 & 1 / 3 & 1 / 3 \\
0 & 0 & 0 \\
-1 / 3 & -1 / 3 & -1 / 3
\end{array}\right|
$$




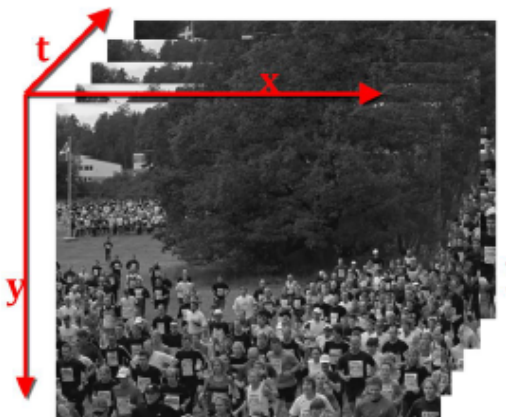

(a) Video Frames

(represented by $x$ and $y$ coordinates)

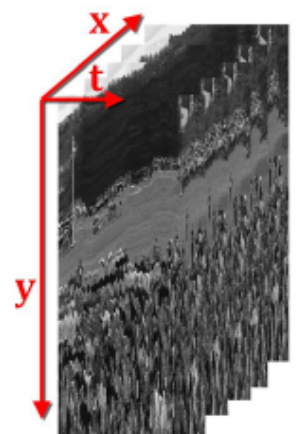

(b) Vertical Slices (represented by $y$ and $t$ coordinates)

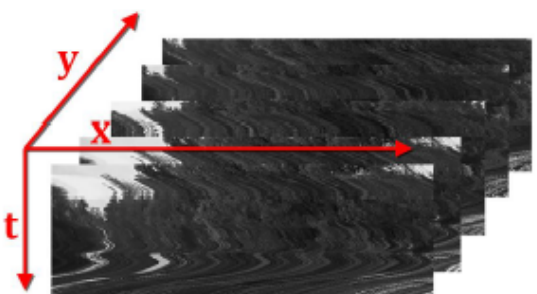

(c) Horizontal Slices

(respresented by $x$ and $t$ coordinates)

Figure 3.3: Example of Spatial Temporal Slices. Originals taken from [2].

To compute the gradient magnitude image, we convolve the reference and distorted (test) images with the Prewitt filters, as shown in the following equations:

$$
m_{r}=\sqrt{\left(I_{r e f} * h_{x}\right)^{2}+\left(I_{r e f} * h_{y}\right)^{2}}
$$

and

$$
m_{d}=\sqrt{\left(I_{\text {dist }} * h_{x}\right)^{2}+\left(I_{\text {dist }} * h_{y}\right)^{2}},
$$

where $I_{\text {ref }}$ is the reference image, $I_{\text {dist }}$ is the distorted image, $m_{r}$ is the gradient magnitude of the reference image, $m_{d}$ is the gradient magnitude of the distorted image, and $*$ represents the convolution operation.

Finally, we compare the gradient magnitude of the reference and distorted images using the following equation:

$$
G M S_{\text {map }}=\frac{2 m_{r} m_{d}+c}{m_{r}^{2}+m_{d}^{2}+c}
$$

where $\mathrm{c}$ is a small constant necessary to avoid instability. The GMSD final score is obtained by calculating the standard deviation of the $G M S_{\text {map }}$. To use GMSD as a video quality method we calculate the GMSD for each video frame and average these to obtain the video quality score.

\subsubsection{SSTS-GMSD}

The Spatial and Spatial-Temporal Slices Gradient Magnitude Similarity Deviation (SSTSGMSD) is a full-reference video quality assessment method based on GMSD, which was developed by Yan et al. [2]. It uses Spatial-Temporal Slices (STS) of the video to provide temporal information to the GMSD. A video can be represented as a 3-D array, $F(x, y, t)$, where the $x$ and $y$ are spatial coordinates and the $t$ is a temporal coordinate. Figure 3.3, depicts the STSs. Notice that there are two types of STS: the vertical STS, represented by $y$ and $t$ coordinates, and the horizontal STS represented by $x$ and $t$ coordinates.

The SSTS-GMSD algorithm computes the GMSD of the video frames $(x, y)$, the horizontal 
STS $(x, t)$ and the vertical STS $(y, t)$, as shown in Figure 3.4. Then, the GMSD scores are sorted in descending order and the average value of the first $20 \%$ GMSD scores is computed, for each of these dimensions. This creates the Spatial GMSD index (S-GMSD), the Horizontal-Slice index (H-GMSD), and the Vertical-Slice GMSD index (V-GMSD). The following equation shows how these indices are combined into a single index that represents the quality of the video:

$$
S S T S-G M S D=\sqrt{S-G M S D \cdot V-G M S D \cdot H-G M S D} .
$$

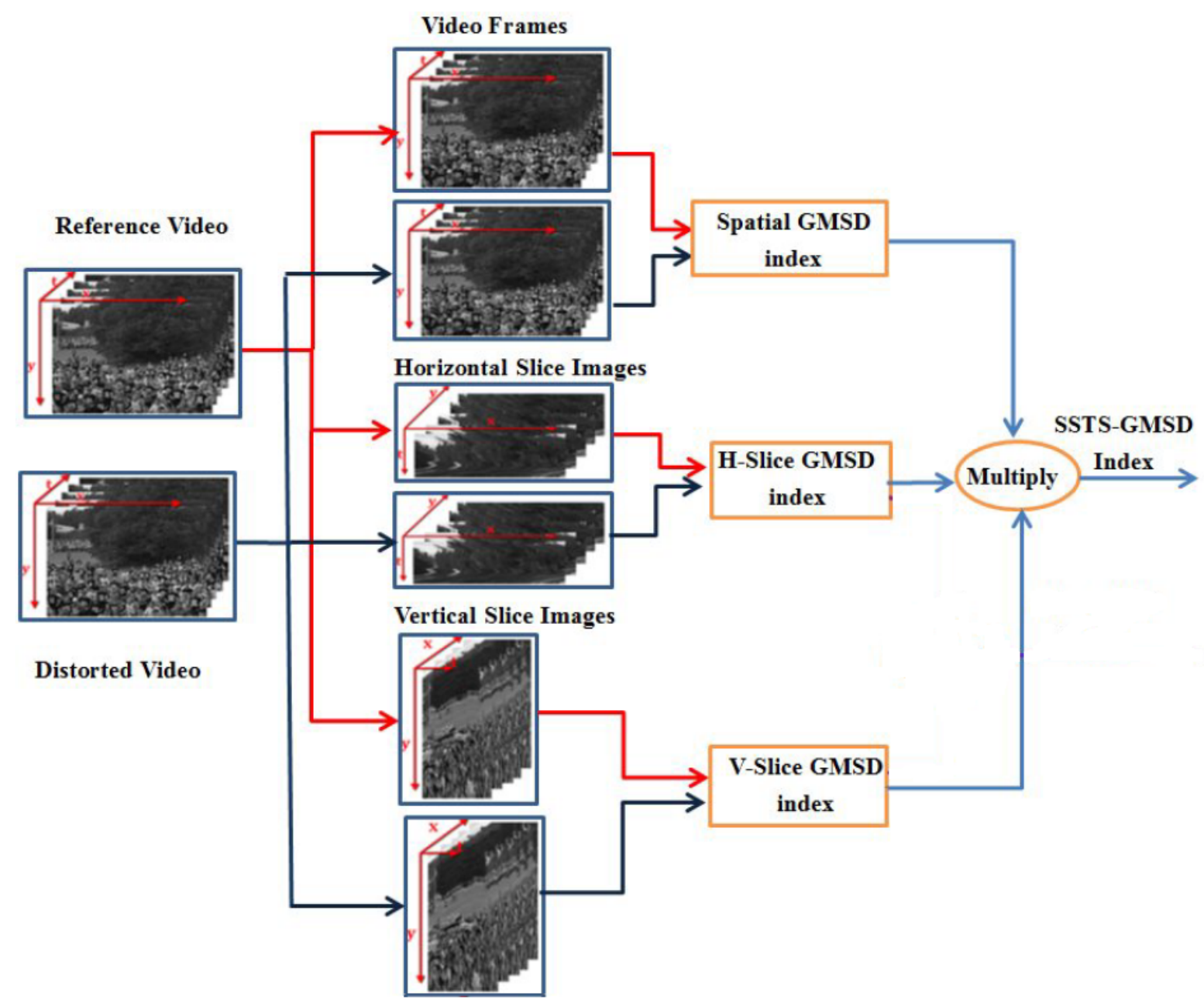

Figure 3.4: Diagram of SSTS-GMSD. Original from [2].

\subsubsection{STRRED}

The Spatial-Temporal Reduced Reference Entropy Difference (STRRED) is a reduced reference video quality method developed by Soundararajan and Bovik [40]. It is based on a previously work, the Reduced Reference Entropic Differencing (RRED) [41]. RRED is a reduced-reference image quality assessment method based on the differences between the entropies of wavelet coefficients of the reference and distorted images. STRRED estimates video quality taking into 
account spatial and temporal distortions. The spatial distortions are measured using the Spatial Reduced Reference Entropic Differences (SRRED). SRRED is based on the frame's RRED index with the addition of a motion weight, what makes it more sensitive to distortions that occur in slow motion regions. Temporal distortions are measured using Temporal Reduced Reference Entropic Differences (TRRED), which is based on the adjacent frames difference RRED index.

The SRRED algorithm calculates the entropic differences of the wavelet coefficients between reference and distorted frames. First, the wavelet coefficients are calculated using a steerable pyramid decomposition with multiple scales and orientations [3]. Figure 3.5 shows an example of a steerable pyramid decomposition in three scales and three orientations. Each image in the steerable pyramid decomposition is considered a sub-band.

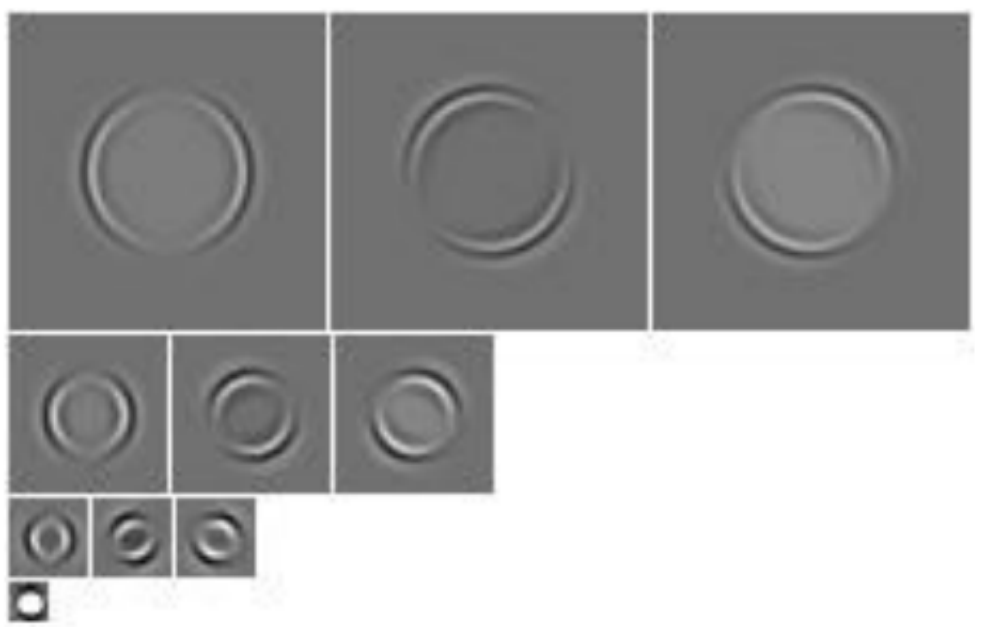

Figure 3.5: Result of steerable pyramid decomposition in three scales and three orientations. Three sub-bands images at each scale and the final lowpass image. Original from [3].

The wavelet coefficient in a sub-band is partitioned in non-overlapping blocks of size $3 \times 3$. Each block in a sub-band $k(k \in 1,2,3, \ldots, k)$ is indexed by $m(m \in 1,2, \ldots, M k)$. Let $\overline{\mathbf{C}}_{m k f}$ denote a vector of wavelet coefficients in the $m_{t h}$ block, the $k_{t h}$ sub-band, and the $f_{t h}$ frame. The vector of wavelet coefficients from the reference video, $\overline{\mathrm{C}}_{m k f r}$, is modeled as a continuous Gaussian Scale Mixture (GSM) distribution [42], defined by the following equation:

$$
\overline{\mathrm{C}}_{m k f r}=S_{m k f r} \overline{\mathrm{U}}_{m k f r}
$$

where $S_{m k f r}$ are independent of $\overline{\mathbf{U}}_{m k f r}$, with $\overline{\mathbf{U}}_{m k f r} \sim \mathcal{N}\left(0, \mathrm{~K}_{U} k f\right)$. The vectors of wavelet coefficients from the distorted video, $\overline{\mathbf{C}}_{m k f d}$, are modeled in the same way.

SRRED is calculated with the following equation:

$\operatorname{SRRED} D_{k}^{M_{k}}=\frac{1}{F M_{k}} \sum_{f=1}^{F} \sum_{m=1}^{M_{k}}\left|\gamma_{m k f r} h\left(C_{m k f r}^{\prime} \mid S_{m k f r}=s_{m k f r}\right)-\gamma_{m k f d} h\left(C_{m k f d}^{\prime} \mid S_{m k f d}=s_{m k f d}\right)\right|$,

where $F$ is the total of frames in the video, $M_{k}$ is the total number of blocks in the sub-band, $h\left(C_{m k f r}^{\prime} \mid S_{m k f r}=s_{m k f r}\right)$ and $h\left(C_{m k f d}^{\prime} \mid S_{m k f d}=s_{m k f d}\right)$ are the entropies of $C_{m k f r}^{\prime}$ and $C_{m k f d}^{\prime}$, 
which are conditioned on the maximum likelihood estimates $S_{m k f r}$ and $S_{m k f d}$. Finally, $\gamma_{m k f r}$ and $\gamma_{m k f d}$ are scale factors defined by the following equations:

$$
\gamma_{m k f r}=\log \left(1+s_{m k f r}^{2}\right)
$$

and

$$
\gamma_{m k f d}=\log \left(1+s_{m k f d}^{2}\right)
$$

The TRRED algorithm is based on the entropic differences of the wavelet coefficients of the current frame and the next frame, in both the reference and distorted videos. Let $\overline{\mathrm{D}}_{m k f}$ denote the vector of wavelet coefficients differences of the current frame $(f)$ and the next frame $(f+1)$, corresponding to the $m_{t h}$ block and the $k_{t h}$ sub-band. The block $\overline{\mathrm{D}}_{m k f r}$, from the reference video, is modeled as a continuous GSM distribution [42], as defined by the following equation:

$$
\overline{\mathrm{D}}_{m k f r}=T_{m k f r} \overline{\mathrm{V}}_{m k f r}
$$

where $T_{m k f r}$ is independent of $\overline{\mathrm{V}}_{m k f r}$, with $\overline{\mathrm{V}}_{m k f r} \sim \mathcal{N}\left(0, \mathrm{~K}_{V} k f\right)$. The vectors of the wavelet coefficients differences of the current and next distorted frames are modeled in the same way, and defined as $\overline{\mathrm{D}}_{m k f d}$.

TRRED is calculated using the following equation:

$T R R E D_{k}^{M_{k}}=\frac{1}{F M_{k}} \sum_{f=1}^{F} \sum_{m=1}^{M_{k}}\left|\delta_{m k f r} h\left(D_{m k f r}^{\prime} \mid T_{m k f r}=t_{m k f r}\right)-\delta_{m k f d} h\left(D_{m k f d}^{\prime} \mid T_{m k f d}=t_{m k f d}\right)\right|$,

where $F$ is the total of frames in the video, $M_{k}$ is the total number of blocks in the sub-band, $h\left(D_{m k f r}^{\prime} \mid T_{m k f r}=t_{m k f r}\right)$ and $h\left(D_{m k f d}^{\prime} \mid T_{m k f d}=t_{m k f d}\right)$ are the entropies of $D_{m k f r}^{\prime}$ and $D_{m k f d}^{\prime}$, which are conditioned on the maximum likelihood estimates of $T_{m k f r}$ and $T_{m k f d}$. Finally, $\delta_{m k f r}$ and $\delta_{m k f d}$ are scale factors defined by:

$$
\gamma_{m k f r}=\log \left(1+s_{m k f r}^{2}\right) \log \left(1+t_{m k f r}^{2}\right)
$$

and

$$
\gamma_{m k f d}=\log \left(1+s_{m k f d}^{2}\right) \log \left(1+t_{m k f d}^{2}\right)
$$

SRRED and the TRRED is combined into a single STRRED quality index. For the $k_{t h}$ subband, the STRRED index is defined as:

$$
S T R R E D_{k}=S R R E D_{k} \cdot T R R E D_{k}
$$

Although the video is decomposed in many sub-bands. These sub-bands have different orientations and size. STRRED is only computed for the sub-band with the vertical orientation and 1/4 of the video size, because this gives the best prediction accuracy performance among all different 


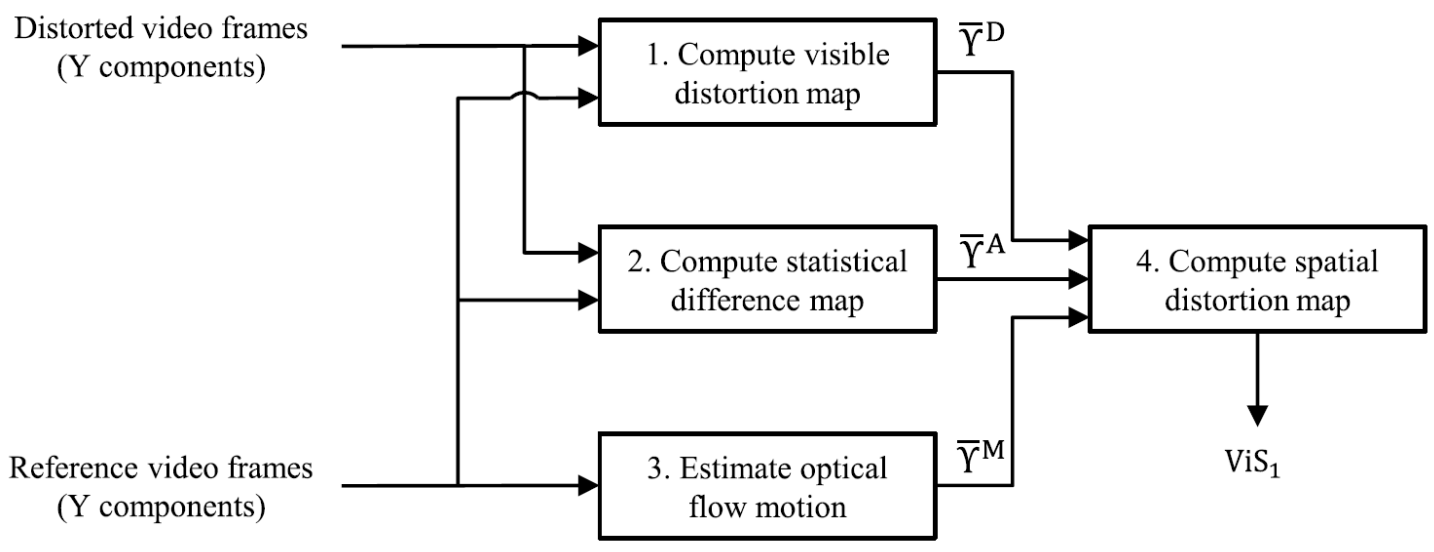

Figure 3.6: Diagram of ViS1 Algorithm. Original from [4].

orientations and scales.

\subsubsection{ViS3}

Video Quality Assessment via Analysis of Spatial and Spatial-Temporal Slices (ViS3) is a full-reference video quality assessment method developed by Vu et al. [4]. This method computes video quality in two stages. First, it estimates the degradations due to spatial distortions. This stage is called ViS1. The second stage, ViS2, estimates the degradations due to joint spatial and temporal distortions.

Figure 3.6 shows an overview of the ViS1 algorithm. To compute the visible distortion map, the reference frame and the distorted frame are converted to perceived luminance values using the following equation:

$$
L=(\alpha+k I)^{\frac{\gamma}{3}}
$$

where $\mathrm{I}$ is the video frame, $\alpha=0, k=0.02874$ and $\gamma=2.2$. These parameters are adjusted for 8-bit pixel values and sRGB displays. An error frame $(\Delta L)$ is computed using the following equation:

$$
\Delta L=L_{\text {ref }}-L_{\text {dist }}
$$

where $L_{\text {ref }}$ and $L_{\text {dist }}$ are the reference and distorted frames converted to perceived luminance. Then, a contrast sensitivity function is applied to the reference frames and error frames using the following equation:

$$
\tilde{L}=\mathscr{F}^{-1}[H(u, v) \cdot \mathscr{F}[L]],
$$

where $\mathscr{F}$ and $\mathscr{F}^{-1}$ correspond to the Discrete Fourier Transform (DFT) and the Inverse DFT, and $H(u, v)$ is the Contrast Sensitivity Function in its DFT version, as defined in [37].

After this pre-processing stage, a local Root Mean Squared (RMS) contrast map is computed for the reference frame. The frame is divided in $16 \times 16$ blocks, with a $75 \%$ of overlap among 
neighboring blocks. The RMS contrast is computed for each block using the following equation:

$$
C_{r e f}=\frac{\tilde{\sigma}(b)}{\mu_{r e f}(b)}
$$

where $\mu_{\text {ref }}(b)$ is the average pixel value of block $b$ of $\tilde{L}$, and $\tilde{\sigma}(b)$ is the minimum of the standard deviations of the four non overlapping $8 \times 8$ blocks within block $b$.

The local RMS contrast for the error frame $(\Delta L)$ is computed. The frame is divided in $16 \times 16$ blocks, with a 75\% overlap between neighboring blocks, and the RMS contrast is computed for each block using the following equation:

$$
C_{e r r}(b)= \begin{cases}\frac{\sigma_{e r r}(b)}{\mu_{r e f}(b)} & \text { if } \mu_{r e f}(b)>0.5 \\ 0 & \text { otherwise }\end{cases}
$$

where $\mu_{\text {ref }}(b)$ is the standard deviation of the error frame $(\Delta L)$ corresponding to block $b$. This adapted RMS contrast algorithm takes into account the human visual system insensitivity to changes in darker areas, by making the values of darker areas equal to zero.

The local distortion visibility map is computed using the following equation:

$$
\left.\zeta_{(} b\right)= \begin{cases}\ln \left[C_{e r r}(b)\right]-\ln \left[C_{r e f}(b)\right] & \text { if } \ln \left[C_{\text {err }}(b)\right]-\ln \left[C_{r e f}(b)\right]>-5 \\ \ln \left[C_{e r r}(b)\right]+5 & \text { if } \ln \left[C_{\text {err }}(b)\right]>-5 \geq \ln \left[C_{r e f}(b)\right] \\ 0 & \text { otherwise. }\end{cases}
$$

Finally, the visible distortion map is computed using a point-by-point multiplication between the local distortion visibility map $(\zeta)$ and the MSE of the reference and distorted frames, as given by the following equation:

$$
\Upsilon^{D}=\zeta(b) \cdot M S E(b)
$$

The statistical difference map measures the differences between the local statistics of multiscale log-gabor filter response of reference and distorted frames. The reference and distorted frames are filtered with a log-Gabor filter bank (with five scales $s \in 1,2,3,4,5$ and four orientations $o \in 1,2,3,4)$. The result are sets of log-Gabor subbands of the reference $\left(R^{s, o}\right)$ and distorted $\left(\hat{R}^{s, o}\right)$ frames. Each log-Gabor subband is divided in $16 \times 16$ blocks, with a $75 \%$ overlapping between neighboring blocks. Then, the standard deviation, skewness, and kurtosis are computed for each block. The statistical difference of the block $b$ is computed as:

$$
\Upsilon^{A}(b)=\sum_{s=1}^{5} \sum_{o=1}^{4} w_{s}\left[\left|\sigma^{s, o}(b)-\hat{\sigma}^{s, o}(b)\right|+2\left|\zeta^{s, o}(b)-\hat{\zeta}^{s, o}(b)\right|+\left|\kappa^{s, o}(b)-\hat{\kappa}^{s, o}(b)\right|\right],
$$

where $\sigma^{s, o}(b), \zeta^{s, o}(b)$ and $\kappa^{s, o}(b)$ are the standard deviation, skewness and kurtosis of block $b$ and subband $\left(R^{s, o}\right)$, respectively. And $\hat{\sigma}^{s, o}(b), \hat{\zeta}^{s, o}(b)$ and $\hat{\kappa}^{s, o}(b)$ are the standard deviation, skewness and kurtosis of block $b$ and subband $\left(\hat{R}^{s, o}\right)$. 
Motion vectors are computed to model the effects of motion on video quality. In areas with larges amount of movement, distortions are less visible. Alternatively, in areas with less motion, distortions are more visible. Motion vectors are computed using the Lucas-Kanade method [43]. In this method, two matrices of motion vectors are obtained, $M_{v}$ for the vertical motion and $M_{h}$ for the horizontal motion. The motion magnitude matrix is computed as:

$$
M=\sqrt{M_{v}^{2}+M_{h}^{2}}
$$

The visible distortion map, statistical difference map, and the motion magnitude map are computed for a Group of Video Frames (GOF). The point-by-point average value of these maps represents the map values for the GOF, given by the following equations:

$$
\begin{aligned}
& \bar{\Upsilon}_{k}^{D}=\frac{1}{N} \sum_{\tau=1}^{N} \bar{\Upsilon}_{N(k-1)+\tau}^{D} \\
& \bar{\Upsilon}_{k}^{A}=\frac{1}{N} \sum_{\tau=1}^{N} \bar{\Upsilon}_{N(k-1)+\tau}^{A} \\
& \bar{\Upsilon}_{k}^{M}=\frac{1}{N} \sum_{\tau=1}^{N} \bar{M}_{N(k-1)+\tau}
\end{aligned}
$$

where $N$ is the number of frames in the GOF. ViS3 uses $N=8$.

Finally, ViS1 is a combination of the visible distortion map, statistical difference map and motion magnitude map, given by:

$$
V i S 1=\frac{1}{K} \sum_{k=1}^{K} \sqrt{\frac{1}{W H} \sum_{x=1}^{W} \sum_{y=1}^{H} \frac{\left[\bar{\Upsilon}_{k}^{D}(x, y)\right]^{\left.\hat{\alpha}(x, y) \cdot \bar{\Upsilon}_{k}^{A}(x, y)\right]^{1-\hat{\alpha}(x, y)}}}{\sqrt{1+\bar{\Upsilon}_{k}^{M}(x, y)}}}
$$

where $\hat{\alpha}(x, y)$ is a parameter given by the following equation:

$$
\hat{\alpha}(x, y)=\frac{1}{1+\beta_{1} \cdot\left[\bar{\Upsilon}_{k}^{D}(x, y)\right]^{\beta_{2}}},
$$

where $\beta_{1}=0.46711$ and $\beta_{2}=0.12964$. Basically, the ViS1 score is a weighted product of the visible distortion map and the statistical difference map, divided by the motion magnitude map.

In ViS2, the frames are converted to the perceived luminance, as shown in Equation 3.22. The spatial-temporal slices are extracted from the videos, similarly to what is done in SSTSGMSD. Let $S_{x}(t, y)$ and $\hat{S}_{x}(t, y)$ denote the vertical slices of the reference and distorted videos, and $x \in[1, W]$, where $W$ is the video spatial width. $S_{y}(x, t)$ and $\hat{S}_{y}(x, t)$ denote the horizontal slice of the reference and distorted video, respectively, and $y \in[1, H]$, where $W$ is the video spatial height. Figure 3.7 shows an overview of the ViS2 algorithm. First, the spatial-temporal correlation map and spatial-temporal response difference map are computed. Then, both maps are combined to compose the spatial-temporal dissimilarity map. 


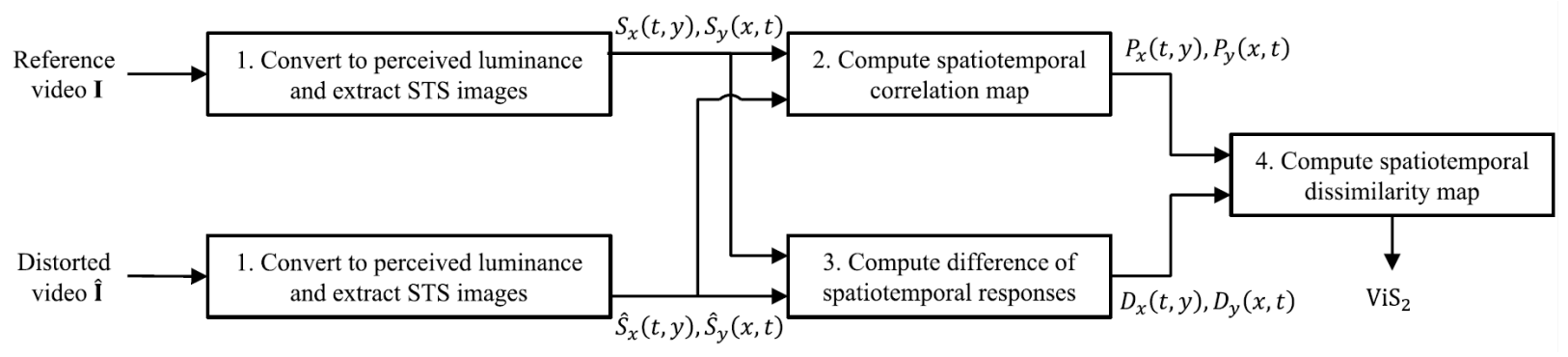

Figure 3.7: Diagram of ViS2 Algorithm. Original from [4].

To compute the spatial-temporal correlation map, the spatial-temporal slice is divided in $16 \times 16$ blocks, with a $75 \%$ overlap among neighboring blocks. Local linear correlation coefficients of the spatial-temporal slices are extracted from the reference and distorted videos, respectively, as defined in the following equation:

$$
\tilde{\rho}(b)= \begin{cases}0, & \text { if } \rho(b)<0 \\ 1, & \text { if } \rho(b)>0.9 \\ \rho(b) & \text { otherwise }\end{cases}
$$

where $\rho$ is the linear correlation coefficient and $b$ is the block of the spatial-temporal slice.

A spatial-temporal response map is computed using separate 1-D filters to each dimension of the spatial-temporal slice. The spatial filter is a set of log-Gabor 1-D filters, $g_{s}$, with $s \in$ $1,2,3,4,5$. The frequency response of the filter is defined in the following equation:

$$
G_{s}(\omega)=\exp \left[-\frac{\left(\ln \left|\frac{\omega}{\omega_{s}}\right|\right)^{2}}{2\left(\ln B_{s}\right)^{2}}\right],
$$

where $\omega_{s}$ is the center frequency of the filter $g_{s}, B_{s}$ is the bandwidth of the filter $g_{s}$ and $\omega \in$ $\left[-\omega_{s}, \omega_{s}\right]$ is the 1-D spatial frequency.

The two temporal filters $h_{z}, z \in 1,2$ are defined as:

$$
h_{z}(t)=t^{n_{z}} \exp (-t)\left[\frac{1}{n_{z} !-\frac{t^{2}}{\left(n_{z}+2\right) !}}\right],
$$

where $n_{1}=6$ and $n_{2}=9$. The spatial-temporally filtered images obtained by filtering $S_{x}(t, y)$ are given by:

$$
R_{x}^{s, z}(t, y)=\left[S_{x}(t, y) *^{y} g_{s}\right] *^{t} h_{z},
$$

The spatial-temporally filtered images obtained by filtering $S_{y}(t, y)$ are given by:

$$
R_{y}^{s, z}(x, t)=\left[S_{y}(x, t) *^{x} g_{s}\right] *^{t} h_{z},
$$


where $*^{d}$ is the convolution along dimension $d$ and $d \in x, y, t, s \in 1,2,3,4,5$ and $z \in 1,2$. So, in total, there are 10 spatial-temporal filtered image for each spatial-temporal slice, $S_{x}(t, y)$ and $S_{y}(t, y)$. Similarly, $\hat{R}_{x}^{s, z}$ and $\hat{R}_{y}^{s, z}$ are computed as the spatial-temporally filtered images of $\hat{S}_{x}$ and $\hat{S}_{y}$. The absolute difference of the spatial-temporally filtered images are computed by:

$$
\begin{aligned}
& \Delta R_{x}^{s, z}(t, y)=\left|R_{x}^{s, z}(t, y)-\hat{R}_{x}^{s, z}(t, y)\right| \\
& \Delta R_{y}^{s, z}(x, t)=\left|R_{y}^{s, z}(x, t)-\hat{R}_{y}^{s, z}(x, t)\right|
\end{aligned}
$$

The response difference map is computed as a natural logarithm of a weighted sum of all adjusted standard deviation maps. $D_{x}$ and $D_{y}$ are the log of response difference maps of the vertical and horizontal spatial-temporal slices, respectively, which are computed using the following equations:

$$
\begin{aligned}
& D_{x}(t, y)=\ln \left\{1+A \sum_{s=1}^{5} \sum_{z=1}^{2} w_{s}\left[\tilde{\sigma}_{x}^{s, z}(t, y)\right]^{2}\right\}, \\
& D_{y}(x, t)=\ln \left\{1+A \sum_{s=1}^{5} \sum_{z=1}^{2} w_{s}\left[\tilde{\sigma}_{y}^{s, z}(t, y)\right]^{2}\right\},
\end{aligned}
$$

where $w_{s}$ are weights, $w_{s}=0.5,0.75,1,5,6, A=10^{4}$ is a scaling factor, and $\tilde{\sigma}_{x}^{s, z}$ and $\sigma_{y}^{s, z}$ are maps of adjusted standard deviation. $\tilde{\sigma}_{x}^{s, z}$ and $\sigma_{y}^{s, z}$ are computed by:

$$
\tilde{\sigma}_{x}^{s, z}(b)=\left\{\begin{array}{ll}
0, & \text { if } \mu_{x}^{s, z}<p \\
\sigma_{x}^{s, z}(b) \sqrt{\frac{\mu_{x}^{s, z}(b)}{p+\mu_{x}^{s, z}(b)}} & \text { otherwise }
\end{array},\right.
$$

and

$$
\tilde{\sigma}_{y}^{s, z}(b)=\left\{\begin{array}{ll}
0, & \text { if } \mu_{y}^{s, z}<p \\
\sigma_{y}^{s, z}(b) \sqrt{\frac{\mu_{y}^{s, z}(b)}{p+\mu_{y}^{s, z}(b)}} & \text { otherwise }
\end{array} .\right.
$$

The spatial-temporal dissimilarity value for the vertical and horizontal spatial-temporal slices are computed as the RMS value of a combination of the spatial-temporal correlation map and the $\log$ of the response difference map, as shown in the following equations:

$$
\begin{aligned}
& \bar{\Delta}_{c}^{F Y}=\sqrt{\frac{1}{F H} \sum_{t=1}^{F} \sum_{y=1}^{H}\left[D_{x}(t, y) \cdot \sqrt{1-P_{x}(t, y)}\right]^{2}}, \\
& \bar{\Delta}_{r}^{X T}=\sqrt{\frac{1}{W F} \sum_{x=1}^{W} \sum_{t=1}^{F}\left[D_{y}(x, t) \cdot \sqrt{1-P_{y}(x, t)}\right]^{2}},
\end{aligned}
$$

where $P_{x}$ and $P_{y}$ are the spatial-temporal correlation maps of the vertical and horizontal spatialtemporal slices, $D_{x}$ and $D_{y}$ are the log of the response difference maps of the vertical and hori- 


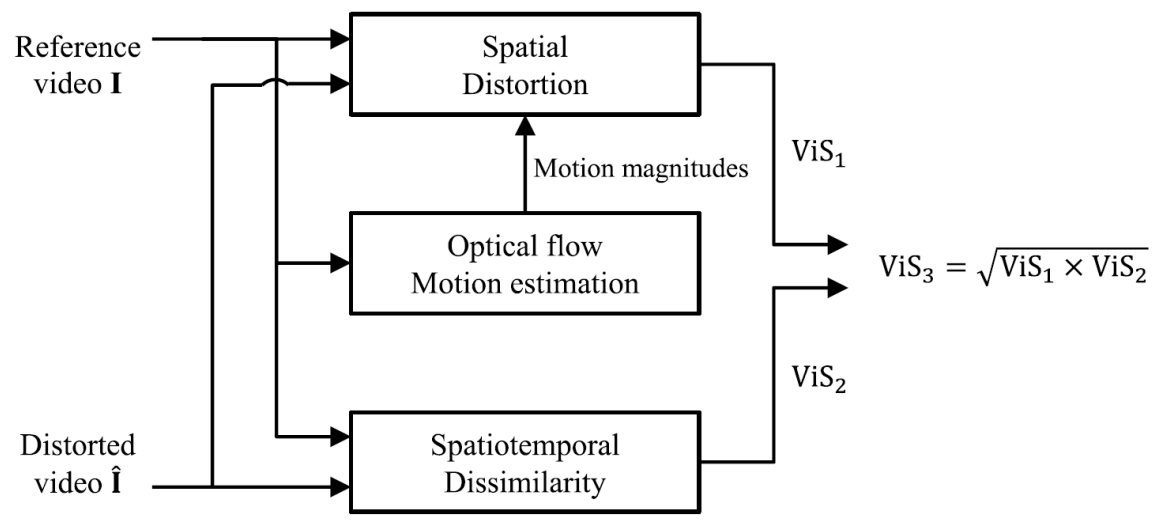

Figure 3.8: Diagram of ViS3 Algorithm. Original from [4].

zontal spatial-temporal slices, $W$ is the width of the video, $H$ is the height of the video and $F$ is the number of frames.

The ViS2 score represents the spatial-temporal dissimilarity value and is computed with the following equation:

$$
V i S 2=\sqrt{\frac{1}{W} \sum_{c=1}^{W}\left[\Delta_{c}^{\bar{F} Y}\right]^{2}+\frac{1}{H} \sum_{H}^{r=1}\left[\Delta_{r}^{\bar{X} F}\right]^{2}} .
$$

Finally, Figure 3.8 shows how to calculate the ViS3 scores, by combining the ViS1 and ViS2 scores into a single scalar. More specifically, ViS3 is the geometric mean of ViS1 and ViS2, given by:

$$
V i S 3=\sqrt{V i S 1 \cdot V i S 2}
$$

The ViS3 score represents the overall perceived video quality.

\subsection{VIDEO QUALITY DATABASES}

Currently, there are several video quality databases available for download. These databases are a collection of videos and their distorted versions. Each distorted video has a subjective score that represents the quality of this video. In most cases, the distorted videos are labeled according to their type of distortion (see previous chapter). In this work, we use four databases as benchmarks to test the proposed video quality assessment framework: LIVE, CSIQ, IVP and MCL-V. In the next subsections, we describe the specifications of each database.

\subsubsection{LIVE Video Quality Assessment Database}

The LIVE Video Quality Assessment Database is one of the most popular video quality database [44, 31]. It was released in 2010 by the Laboratory for Image \& Video Engineering 
(LIVE) of the University of Texas. This database has 10 reference videos and, for each reference video, there are 15 test sequence with four types of distortions: MPEG-2 compression (4 test videos per reference), H.264 compression (4 test videos per reference), simulated transmission of H.264 compressed bitstreams through error-prone IP networks ( 3 test videos per reference) and through error-prone wireless networks (4 test videos per reference). Figure 3.9 shows sample frames from each reference video in the LIVE database.

The videos are provided in uncompressed YUV420 format at a $768 \times 432$ resolution. Nine of the ten reference videos are 10 seconds long and one video is 8.68 seconds long. Seven out of the ten reference videos have 25 FPS and the other three videos have 50 FPS. For the subjective testing, a single stimulus methodology was adopted. In this methodology, the subject watches a single video and then gives a quality score to this video using a continuous scale. All test videos were evaluated by 38 subjects. Subjects evaluated reference and the impaired videos. The Difference Mean Opinion Scores (DMOS) between each impaired video and the corresponding reference was computed to provide a subjective quality score.
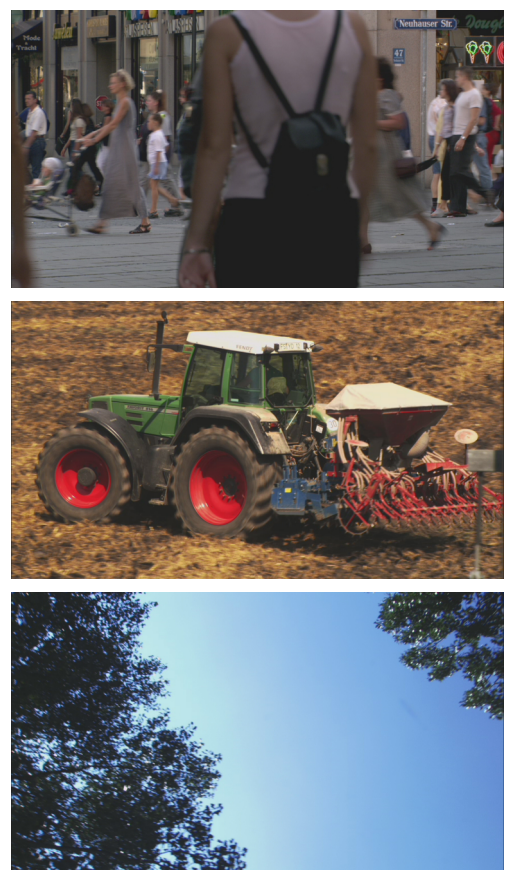
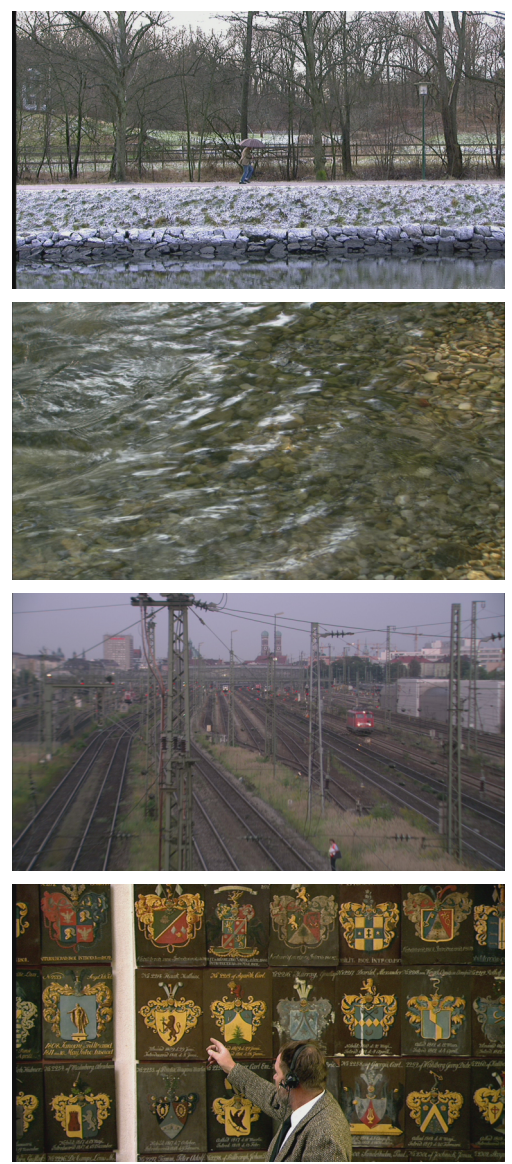
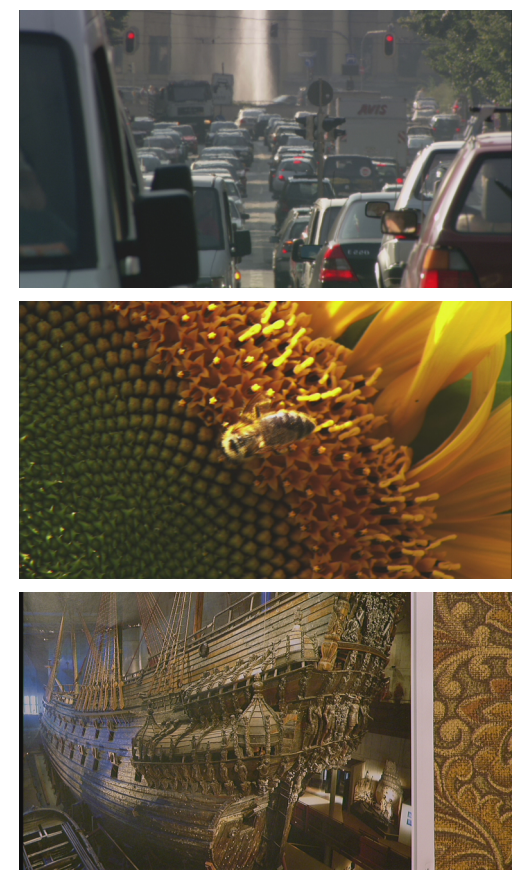

Figure 3.9: Sample frames of original videos in the LIVE Database. 


\subsubsection{CSIQ Video Quality Database}

The CSIQ Video Quality Database was released in 2013 by Laboratory of Computational Perception \& Image Quality at Oklahoma State University [4]. This database contains 12 reference video sequences and 216 distorted video sequences. All videos were provided in uncompressed YUV420 format with a spatial resolution of $832 \times 480$. Figure 3.10 shows sample frames from each reference video in the CSIQ database.

The distorted video sequences were generated using 6 types of distortion: H.264 compression, H.265 compression, MJPEG compression, wavelet-based compression using the Snow codec, H.264 videos subjected to simulated wireless transmission loss, and additive white noise. To generate all test videos, each reference video was distorted with all distortions in three different levels.

The Subjective Assessment Methodology for Video Quality (SAMVIQ) was used in the subjective tests [45]. SAMVIQ is a multi-stimulus experimental methodology. Subjects are presented with the reference and distorted sequences from the same content. They evaluate each sequence in any order. They can even change the quality score given to a sequence previously seen. All videos were evaluated by 35 subjects. The MOS for each video sequence was released with the database.
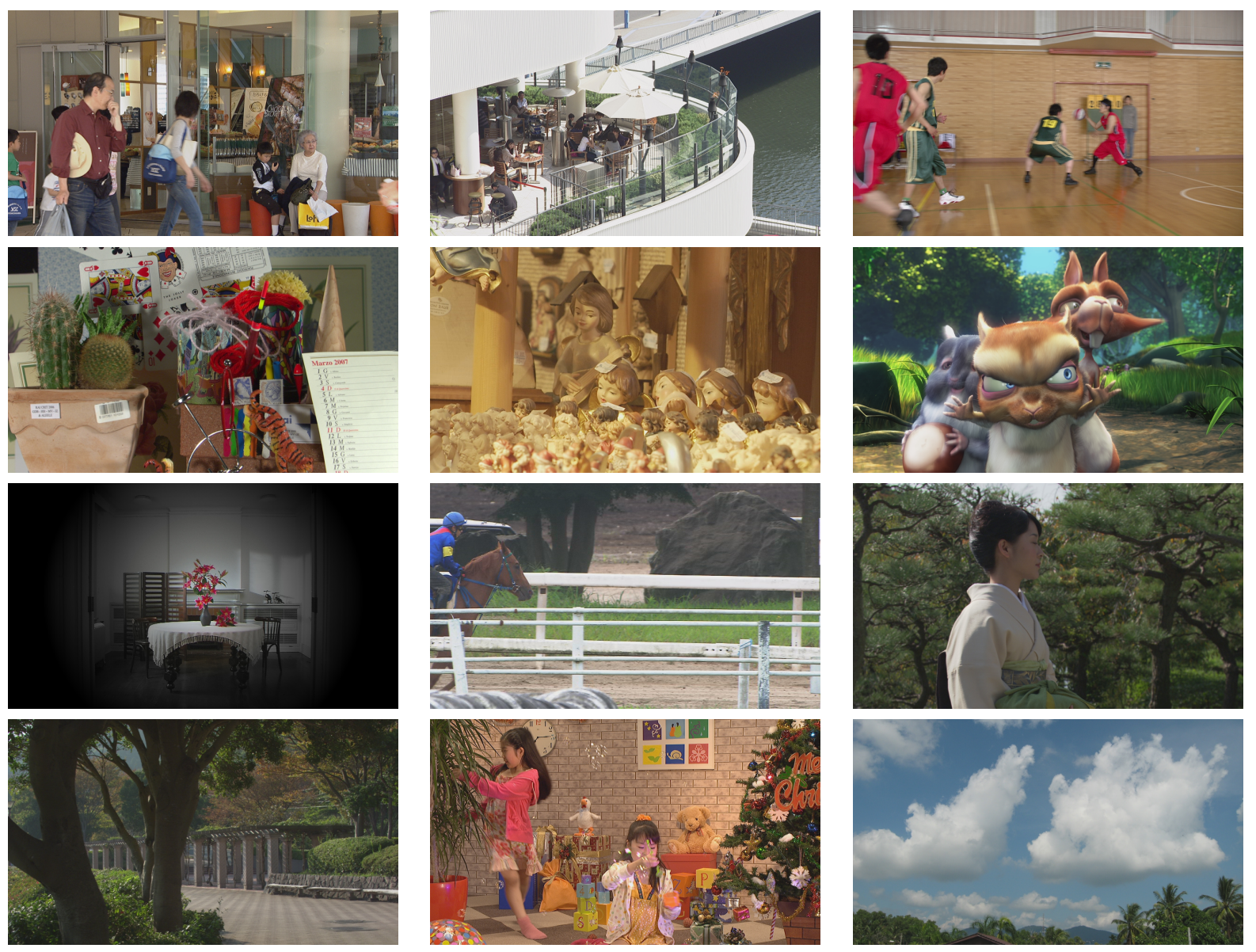

Figure 3.10: Sample frames of original videos in the CSIQ Database. 


\subsubsection{IVP Subjective Quality Video Database}

The IVP Subjective Quality Video Database was released in 2011 by the Image and Video Processing Laboratory at The Chinese University of Hong Kong [46]. This database contains 10 reference video sequences and 128 distorted video sequences. The videos were provided in uncompressed YUV420 format with resolution of 1920x1088. All videos have 25 FPS. Seven of the reference video sequences are 10 seconds long, two are 11.2 seconds long and one is 8.96 seconds long. Figure 3.11 shows sample frames from each reference video in the IVPL database.

The distorted video sequences were generated using 4 types of distortion: MPEG-2 compression (3 test videos per reference), wavelet compression (3 test videos per reference), H.264 compression (4 test videos per reference) and packet loss cause by H.264 streaming through IP networks (4 test videos per reference). The packet loss distortions are presented only for seven (out of ten) reference video sequences. All videos were evaluated by 42 subjects. The single stimulus methodology was used in the subjective experiment. DMOS was provided as the subjective quality measure.
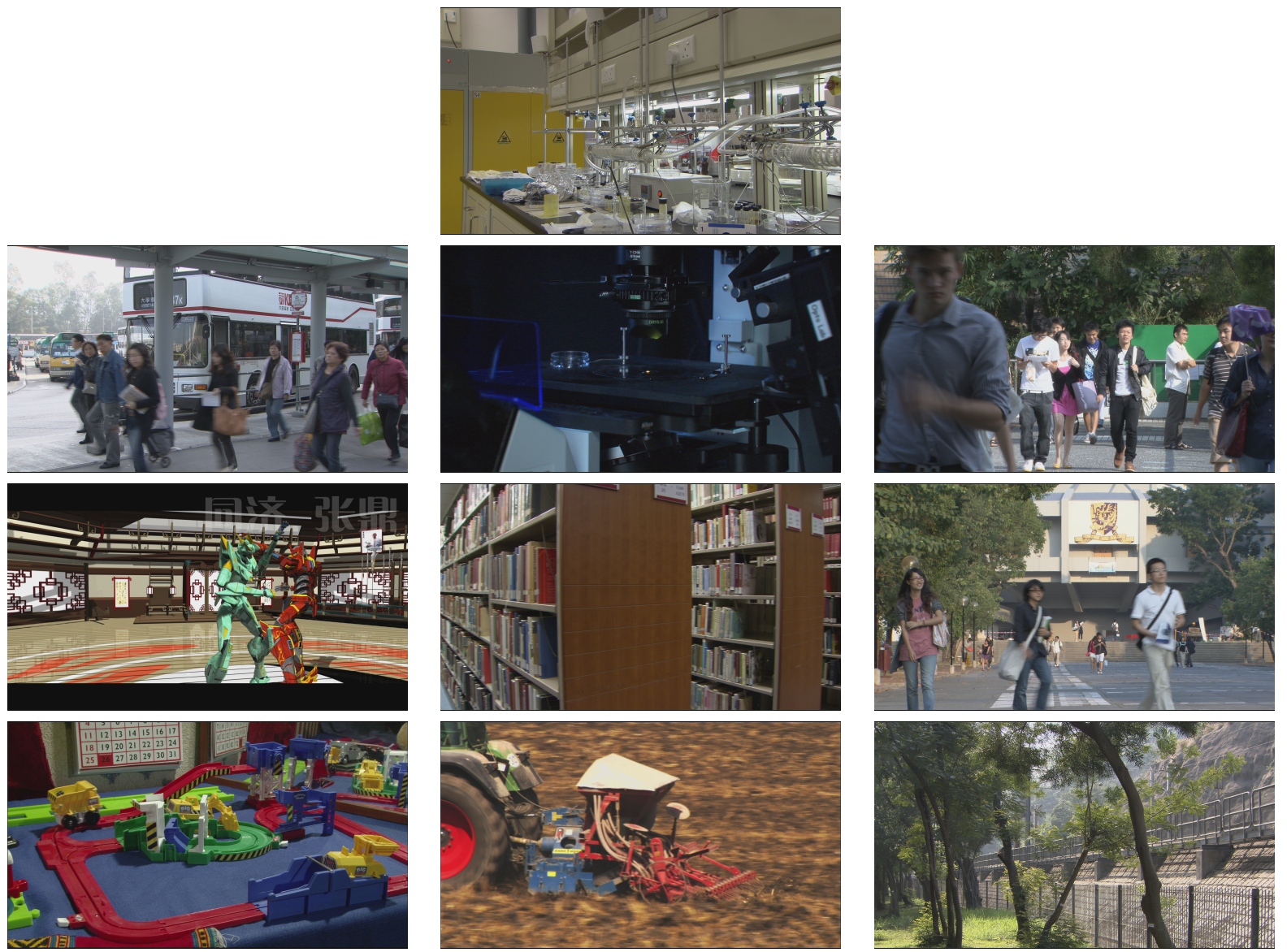

Figure 3.11: Sample frames of original videos in the IVP Database. 


\subsubsection{MCL-V Database}

The MCL-V Database was released in 2014 by the USCMediaCommLab at the University of Southern California [28]. This database contains 12 reference videos sequences and 96 distorted video sequences. The distorted video sequences were generated using 2 types of distortion: H.264 Compression (4 distorted video sequences per reference) and H.264 Compression follow by scaling (4 distorted video sequences per reference). Figure 3.12 shows sample frames from each reference video in the MCL database.

The video sequences are provided in uncompressed YUV420 format with a spatial resolution of $1920 \times 1080$. Two of the reference video sequences have 24 FPS, six of them have 25 FPS and the remaining sequences have 30 FPS. The videos sequences with 25 and 30 FPS are 6 seconds long. One of the video sequences with 24 FPS is 5.5 seconds long and the other video sequence is 5 seconds long.

The videos were evaluated by 45 subjects. A pairwise comparison experimental methodology was used in the subjective test. This methodology is recommended by ITU [47] for detecting small differences in quality. In this methodology the two video sequences (reference and test) from the same source are presented simultaneously. Subjects are asked to choose which video sequence has the best quality. The database provides the MOS for each test sequence.
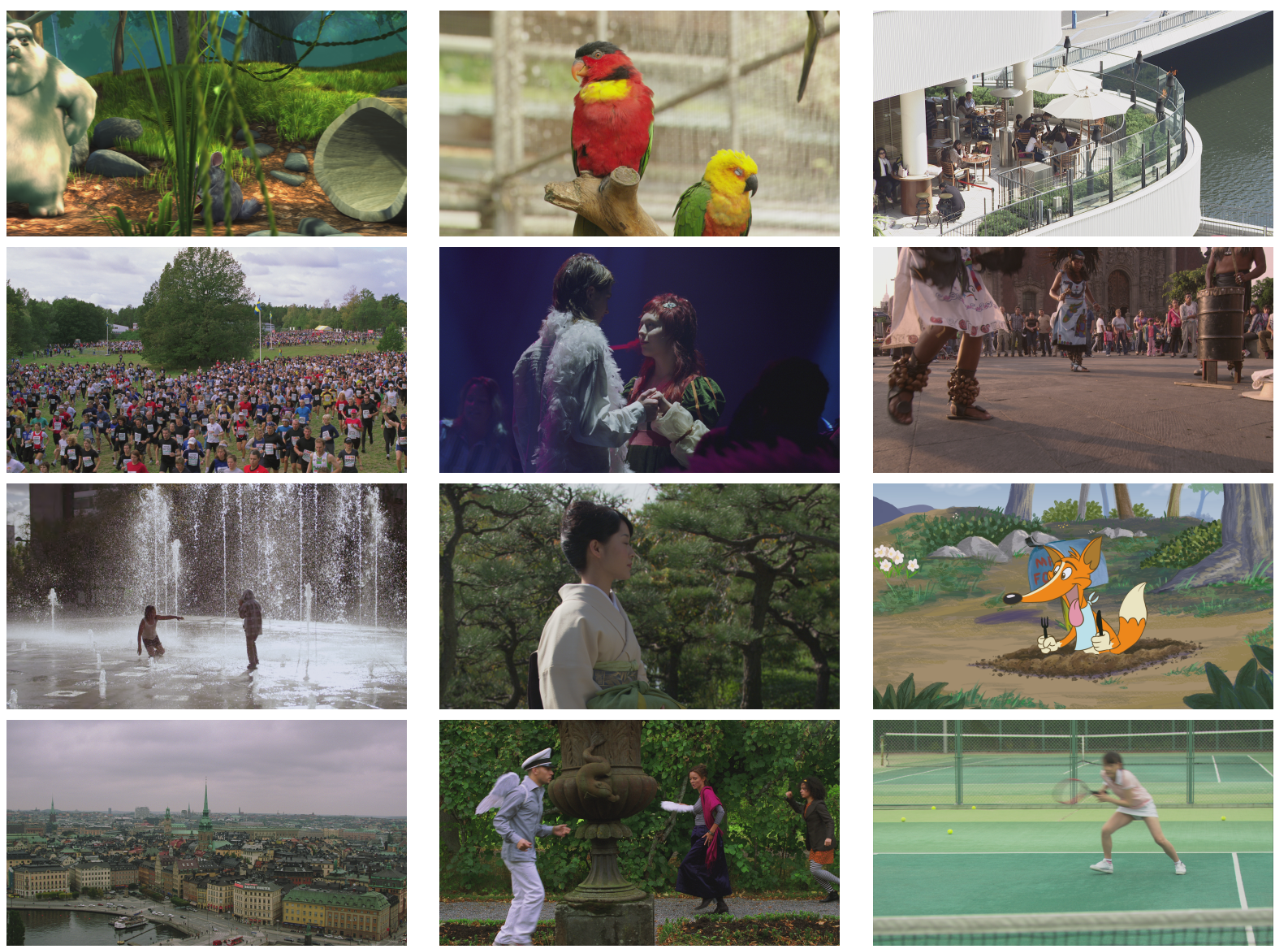

Figure 3.12: Sample frames of original videos in the MCL-V Database. 


\section{PROPOSED FRAMEWORK AND VIDEO CLASSIFIERS}

One of the challenges of video quality assessment methods is to improve the runtime performance of the algorithms [13]. The runtime performance of most of these metrics depends on the video resolution. Therefore, reducing the video spatial resolution improves their runtime. However, accuracy performance can be affected when the spatial resolution is reduced. More specifically, video distortions that affect high frequencies are the most sensitive to video spatial downsampling (MJPEG compression, packet-loss and white noise). Some examples of distortions that are sensitive to downsampling include distortions introduced by MJPEG compression algorithms (e.g. blocking artifacts), packet-loss and noise.

We propose a downsampling video framework that improves the runtime performance of video quality assessment methods by reducing the video resolution, without interfering with the accuracy performance. Before reducing the spatial resolution, the technique identifies the videos that are more sensitive to this reduction in spatial resolution. This framework is illustrated in Figure 4.1.

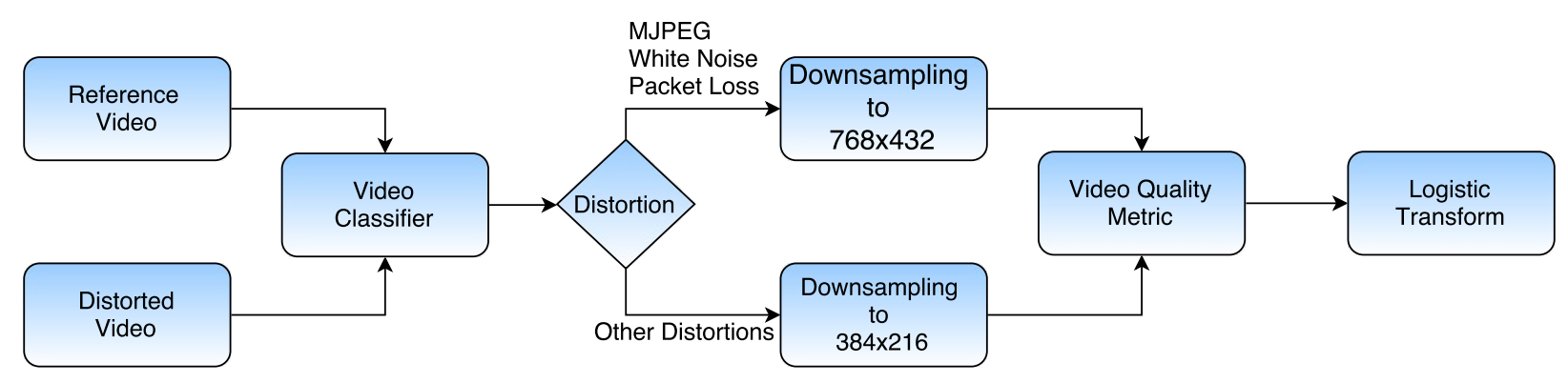

Figure 4.1: High Level Overview of the Downsampling Video Framework.

The framework has four main stages: the video classifier, the video downsampler, the quality assessment method and the logistic transform. The video classifier stage identifies the types of videos more sensitive to a spatial resolution reduction. The downsampler stage reduces the video spatial resolution, accordingly, with the goal of optimizing the resolution according to the video sensitivity. Finally, the logistic transform stage adjusts the predicted scores to a common scale.

We design two different video classifiers that have the goal of identifying the types of distortions in the video and choose the most adequate downsampling ratio. Video classifiers are algorithms that classify videos by analyzing one or more features. Videos can be classified according to their content, distortion type or others characteristics. Next, we describe the two video classifiers proposed in this work: a spatial activity difference classifier and a SSEQ classifier. Next, we describe the downsampling and logistic transform stage of the framework. 


\subsection{SPATIAL ACTIVITY DIFFERENCE CLASSIFIER}

The first proposed video classifier is the spatial activity difference classifier. This classifier is based on a analysis of the Spatial Activity (SA) of the videos [48]. In our work, we have noticed that videos distorted by MJPEG compression, white noise and packet-loss have a higher Spatial Activity than the videos with others types of distortions, when considering video sequences with the same content. This can be observed in Figure 4.2, where the white regions are the regions with a higher SA. Because the frames filtered with Sobel have subtle intensity changes, for a better visualization, we perform a post processing of these frames using a histogram equalization [20]. Blocking and erroneously decoded blocks leave false edges in the frame and the Sobel filter can detect them. Therefore, the idea behind this classifier is to use the SA difference between the reference and distorted videos to separate video distortions in two classes. The first class correspond to videos distorted by white noise, packet-loss and MJPEG compression, while the second class corresponds to videos distorted by H.264, MPEG-2, HEVC, wavelet compressions and upscaling.

\subsubsection{Spatial Activity}

The spatial activity (SA) feature is computed by:

$$
S A\left(t_{n}\right)=R M S\left(\operatorname{Sobel}\left(I\left(t_{n}\right)\right)\right)
$$

where $I\left(t_{n}\right)$ is the luminance component of the video frame $t_{n}, R M S$ is the RMS function over the entire image, and Sobel is the Sobel Filter. The Sobel filter used in this work has two $3 \times 3$ kernels. The first one detects horizontal edges and is given by:

$$
G_{x}=\left|\begin{array}{lll}
-1 & 0 & 1 \\
-2 & 0 & 2 \\
-1 & 0 & 1
\end{array}\right| \text {. }
$$

The second kernel detects vertical edges, given by:

$$
G_{y}=\left|\begin{array}{ccc}
-1 & -2 & -1 \\
0 & 0 & 0 \\
1 & 2 & 1
\end{array}\right| .
$$

Then, the luminance component of the video frame is filtered with the Sobel filter and combined in the following form:

$$
\operatorname{Sobel}\left(t_{n}\right)=\sqrt{\left(G_{x} * I_{n}\right)^{2}+\left(G_{y} * I_{n}\right)^{2}},
$$

where $*$ is the symbol for the convolution and $I_{n}$ is the luminance component of the video frame. 


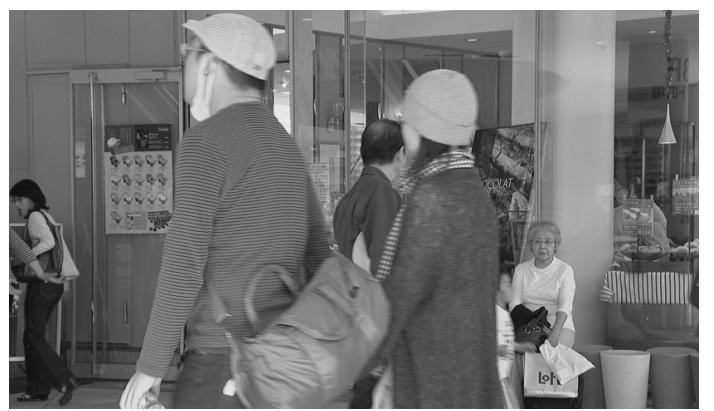

(a) Reference Frame

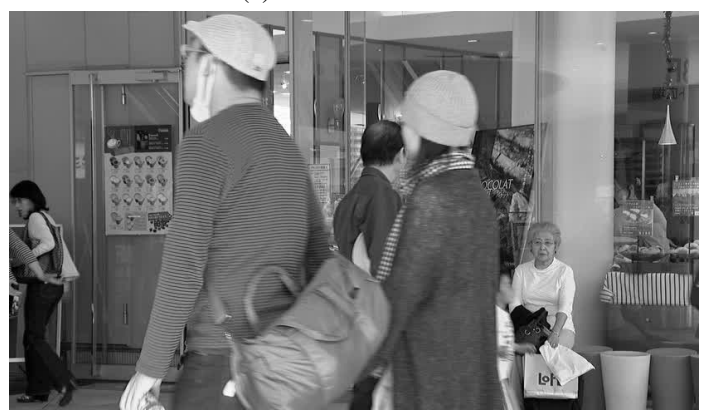

(c) frame compressed with MJPEG

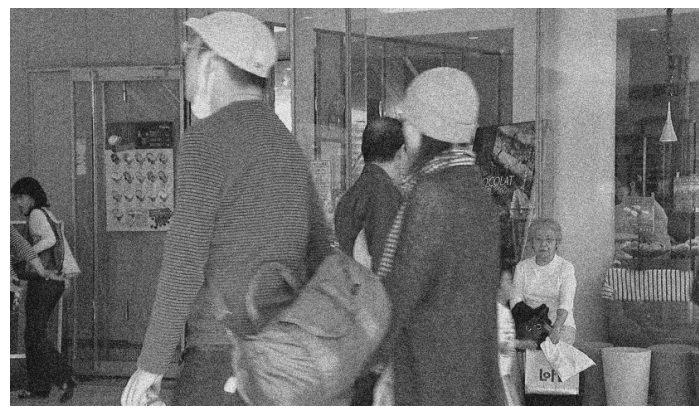

(e) frame corrupted with White Noise

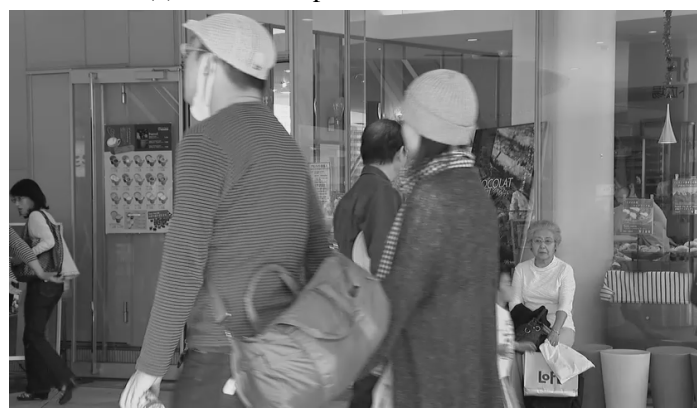

(g) frame compressed with H.264

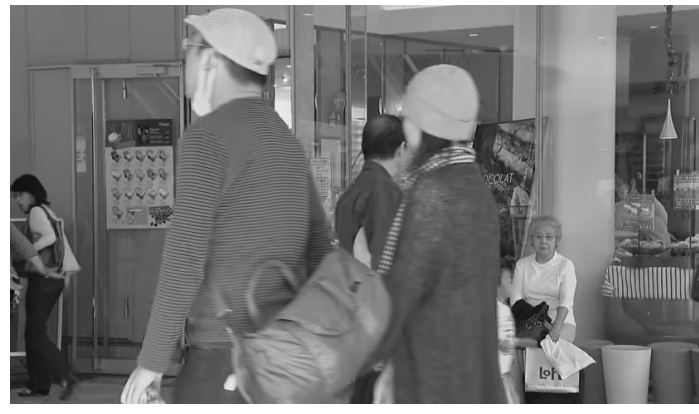

(g) frame compressed with HEVC

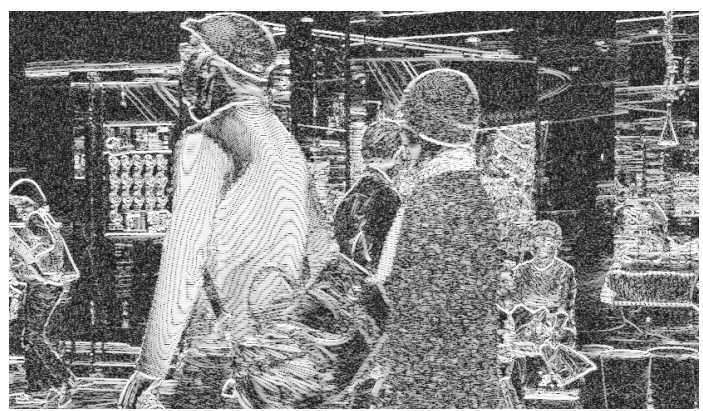

(b) Spatial Activity $=91.11$

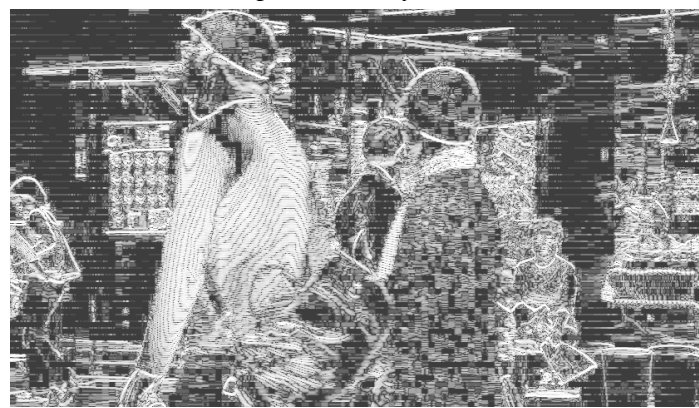

(d) Spatial Activity $=101.38$

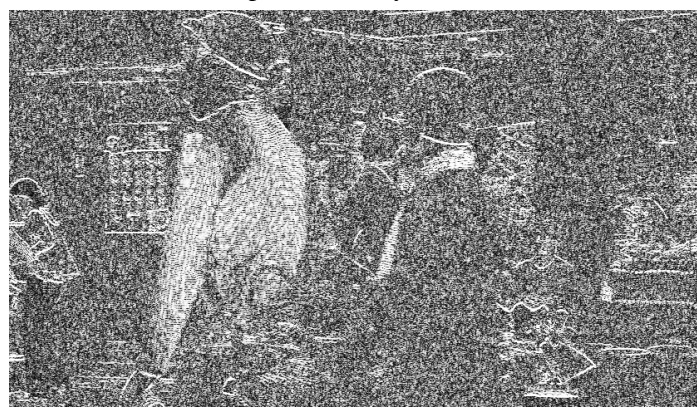

(f) Spatial Activity $=119.76$

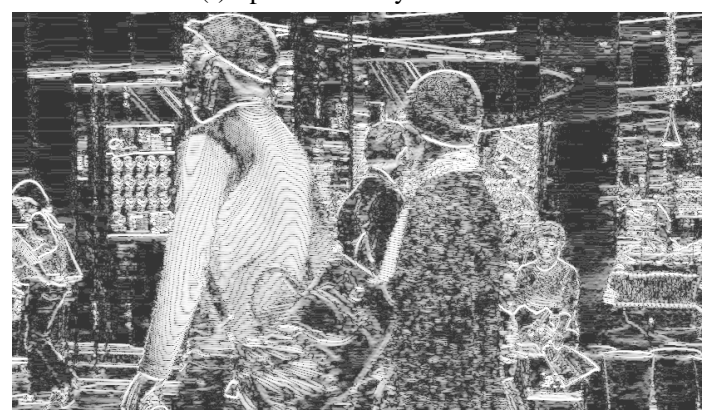

(h) Spatial Activity $=88.75$

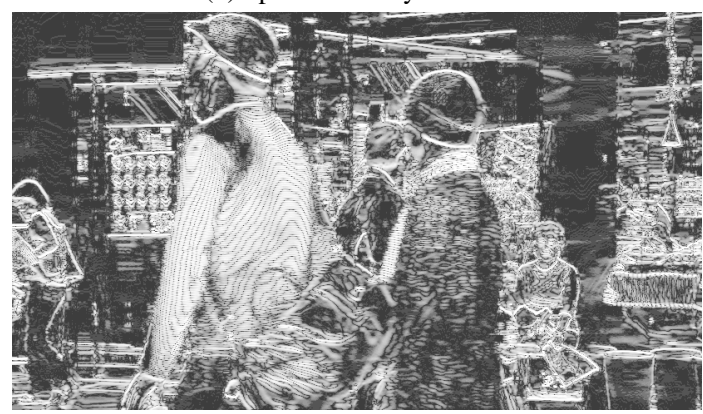

(h) Spatial Activity = 83.71

Figure 4.2: Sample frames (unimpaired and distorted) are shown in the left, while their Sobel filtered versions are shown in the right, along with the spatial activity (SA) measure. 


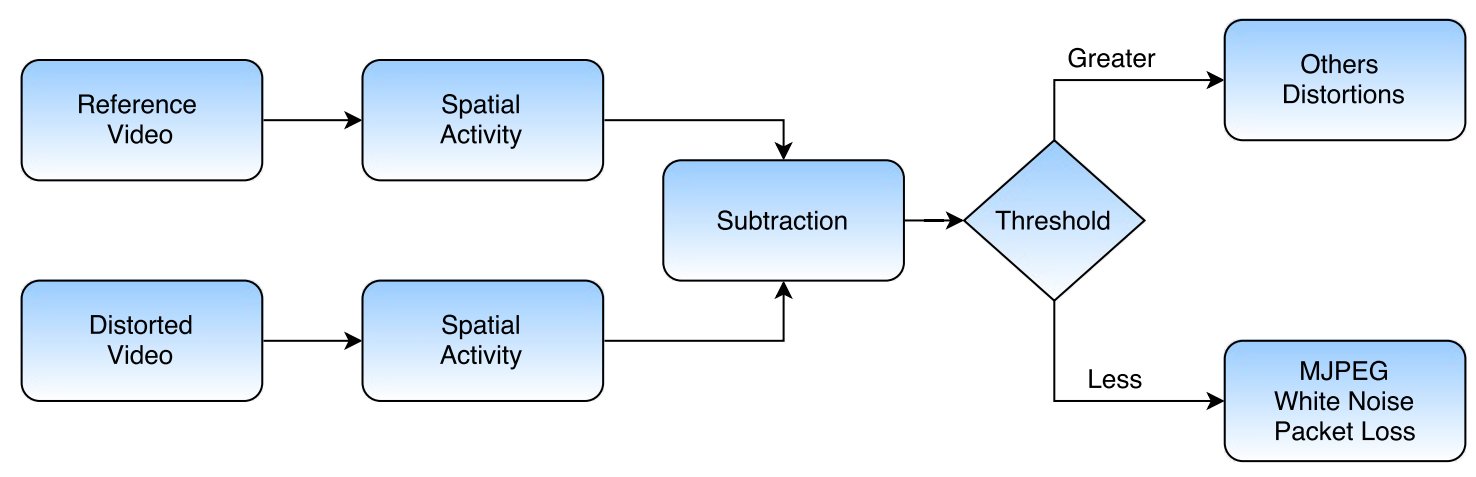

Figure 4.3: Diagram of the proposed Spatial Activity Classifier.

We use the implementation provided by the Monitoring Of Audiovisual Quality by Key Indicators (MOAVI) group [49]. To calculate the video Spatial Activity, we average the SA values of all frames in the video.

\subsubsection{Spatial Activity Difference Algorithm}

Figure 4.3 shows the diagram of the proposed classification method. First, we compute the SA of the reference and distorted videos, and, then, we calculate their difference $(\Delta \mathrm{SA})$. Considering that the videos we are trying to identify have a higher (MJPEG compression and white noise) or a slightly lower space activity (packet-loss) than the reference video, we can use a $\Delta \mathrm{SA}$ threshold to classify these videos.

To identify the best threshold, we tested 9 values of thresholds, with $\Delta$ SA values ranging from -4 to 4 . Then, we use the F1 score, which measures the accuracy of a classifier (see Appendix I), to select the best threshold. The values of the F1 Score varies from 1 (best) to 0 (worst). Table 4.1 shows the results of using the 9 thresholds in all videos of the LIVE, CSIQ, IVPL and MCL-V databases. We tested the classifier performance for videos in the original temporal resolution, as well in four reduced temporal resolutions.

Notice that a threshold lower than 1 does not provides a good. Results show that the best threshold value is 2 , but it is not necessary to analyze all frames of the video sequence to obtain a good classification. In fact, using a temporal resolution of 2 frames per second provides, surprisingly, better classification results than what is obtained by analyzing all frames in the video (original temporal resolution).

Table 4.1: F1 scores using threshold for the Difference in Spatial Activity ( $\Delta \mathrm{SA})$, varying from -4 to 4.

\begin{tabular}{|l|c|c|c|c|c|c|c|c|c|}
\hline & \multicolumn{8}{|c|}{ Spatial Activity Difference $(\Delta \mathrm{SA})$} \\
FPS & -4 & -3 & -2 & -1 & 0 & 1 & 2 & 3 & 4 \\
\hline Original & 0.6145 & 0.6216 & 0.6491 & 0.6752 & 0.7032 & 0.7805 & 0.7957 & 0.7869 & 0.7368 \\
\hline 10 FPS & 0.6109 & 0.6216 & 0.6491 & 0.6784 & 0.7032 & 0.7830 & 0.7957 & 0.7902 & 0.7415 \\
\hline 8 FPS & 0.6145 & 0.6216 & 0.6491 & 0.6816 & 0.7032 & 0.7753 & 0.7997 & 0.7907 & 0.7431 \\
\hline 4 FPS & 0.6145 & 0.6216 & 0.6491 & 0.6752 & 0.7093 & 0.7830 & 0.8076 & 0.7858 & 0.7415 \\
\hline 2 FPS & 0.6145 & 0.6216 & 0.6491 & 0.6784 & 0.7062 & 0.7830 & 0.8042 & 0.7832 & 0.7411 \\
\hline
\end{tabular}




\subsection{SSEQ CLASSIFIER}

The second classification method proposed in this work is based on the Spatial-Spectral Entropy-based Quality (SSEQ), which is a no-reference image quality assessment method proposed by Liu et al. (50). In this work, we use only the SSEQ feature extraction module. In this section, we describe both the SSEQ feature extraction stage and the proposed classifier algorithm.

\subsubsection{SSEQ Feature Extration Stage}

SSEQ uses a 2-stage framework. First, the feature extraction stage extracts features from the image and uses them in a distortion classification stage. Second, a quality prediction stage estimates the video quality [51]. Again, we only use the feature extraction stage of the SSEQ.

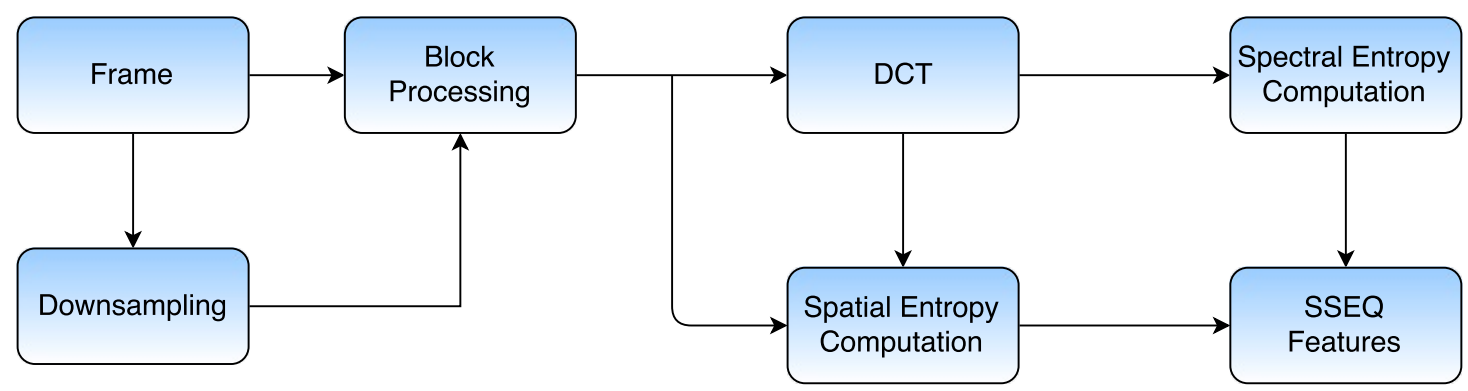

Figure 4.4: Diagram of SSEQ Feature Extraction.

A high-level overview of the SSEQ feature extraction stage is presented in Figure 4.4. First, the frame is downsampled by a factor of 2. A bicubic interpolation method is used in the downsampling step, generating frames with 3 different sizes (1, 0.5, and 0.25 of the original resolution). For each size, the frame is divided into $8 \times 8$ blocks and, then, the spatial and spectral entropies are computed for each block of the frame. The spatial entropy of a block is calculated with the following equation:

$$
E_{s}=-\sum_{M-1}^{x=0} \sum_{N-1}^{y=0} p(x, y) \cdot \log _{2}(p(x, y)),
$$

where $M \times N$ is the block size and $p(x, y)$ is the probability of the pixel intensity value. We compute the spatial entropy $\left(E_{s}\right)$ for each block. Then, we obtain the set of spatial entropy values per block, $S\left(s e_{1}, s e_{2}, \ldots, s e_{m}\right)$, with $s e_{i}$ being the spatial entropy of $i_{t h}$ block.

To compute the spectral entropy, we first obtain the discrete cosine transform (DCT) of each block [52]. Then, we normalize the AC DCT coefficients:

$$
P(u, v)=-\frac{C(u, v)^{2}}{\sum_{M-1}^{x=0} \sum_{N-1}^{y=0} C(u, v)},
$$


where $C(u, v)$ is the DCT coefficient block. The spectral entropy of a block is defined by:

$$
E_{f}=-\sum_{M-1}^{x=0} \sum_{N-1}^{y=0} P(x, y) \cdot \log _{2}(P(x, y)) \text {. }
$$

We compute the spectral entropy $\left(E_{f}\right)$ for each block in the frame. Then, we obtain the set of spectral entropy values per block, $F r\left(f e_{1}, f e_{2}, \ldots, f e_{m}\right)$, with $f e_{i}$ being the spectral entropy of $i_{\text {th }}$ block.

SSEQ uses the percentile pooling as a feature pooling strategy. The percentile pooling technique consists of first sorting the $S$ and $F$ values in ascending order and, then, selecting the $60 \%$ central values. With this, two sets are created: $S_{c}\left(s e_{\lfloor 0.2 m\rfloor}, s e_{\lfloor 0.2 m\rfloor+1}, \ldots, s e_{\lfloor 0.8 m\rfloor}\right)$ and $\operatorname{Fr}_{c}\left(f e_{\lfloor 0.2 m\rfloor}, f e_{\lfloor 0.2 m\rfloor+1}, \ldots, f e_{\lfloor 0.8 m\rfloor}\right)$.

The features for each scale are composed by the average and skewness values of $S_{c}$ and $F r_{c}$ :

$$
f v=\left[\text { mean }\left(S_{c}\right), \operatorname{skewness}(S), \text { mean }\left(F_{c}\right), \text { skewness }(F)\right] .
$$

For each frame, the SSEQ feature vector contains 12 elements, which correspond to the 3 frame sizes and 4 different features.

\subsubsection{SSEQ Classifier Algorithm}

In our work, we extract the SSEQ features of each frame in the video, as described in the earlier section. By the end of the SSEQ feature extraction stage, we have a set of feature vectors $F v=f v_{1}, f v_{2}, \ldots, f v_{n}$, with $f v_{i}$ being the feature vector corresponding to the $i_{t h}$ frame. Next, to create a SSEQ feature vector for the entire video, we average each feature for all video frames, as illustrated in Figure 4.5.

We use a Support Vector Machine (SVM) algorithm to classify image distortions. SVM were first introduce by Cortes and Vapnik as a binary classifier [53]. Given a training set, the SVM can obtain an optimal class separation hyperplane, which is defined as the plane with the maximum margin of separation between the two classes. Also, the margin is the sum of the distances from the hyperplane to the closest point of the two classes. Multiclass problems are solved as a combination of binary problems [54]. We have chosen SVM because of it good performance in several applications, and it is used in the SSEQ image quality assessment method [55, 56, 57].

Figure 4.6 shows an overview of the proposed classifier algorithm. First, we randomly divide the videos in training and testing sets. Then, we extract the SSEQ features of all training videos and label them according to their distortion. Then, we train the SVM with these parameters (SSEQ features and video distortion labels). Then, SVM creates a model that is used to classify others videos in the testing set. In other words, SSEQ features are extracted from a test video. Using the parameters obtained with the trained SVM, we obtain the estimated video distortion label. 


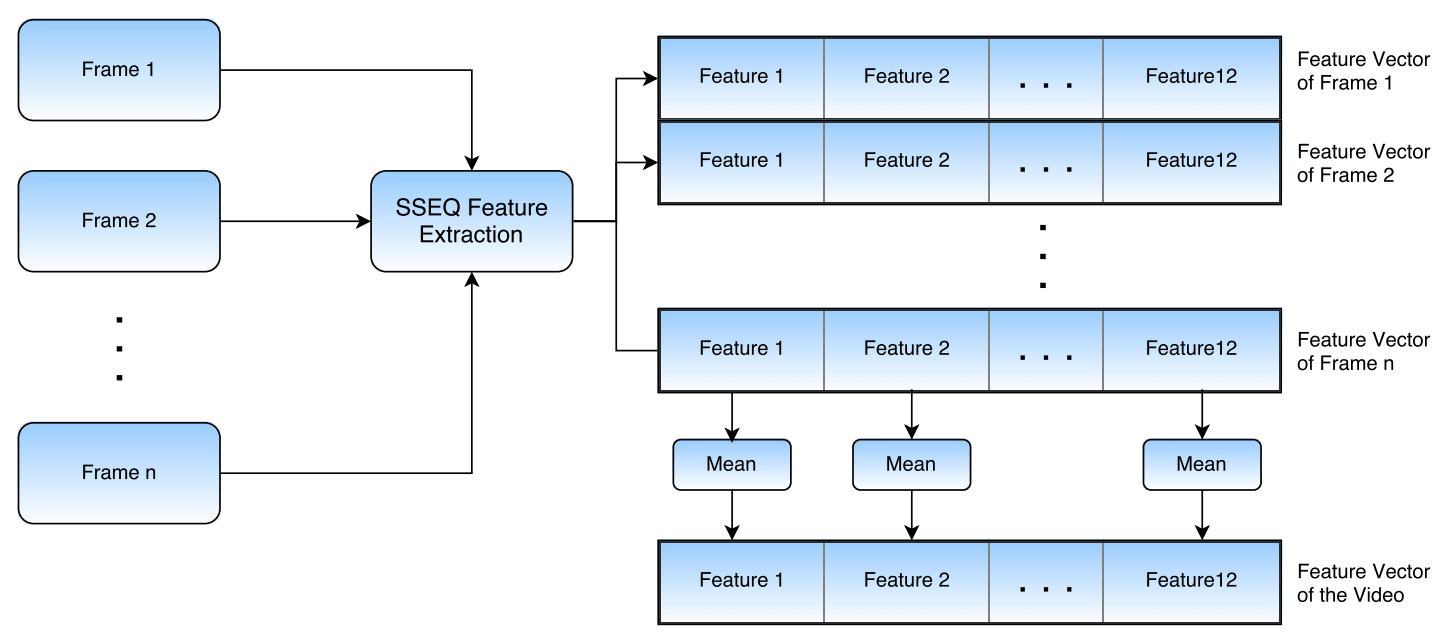

Figure 4.5: SSEQ Video Feature Extraction.

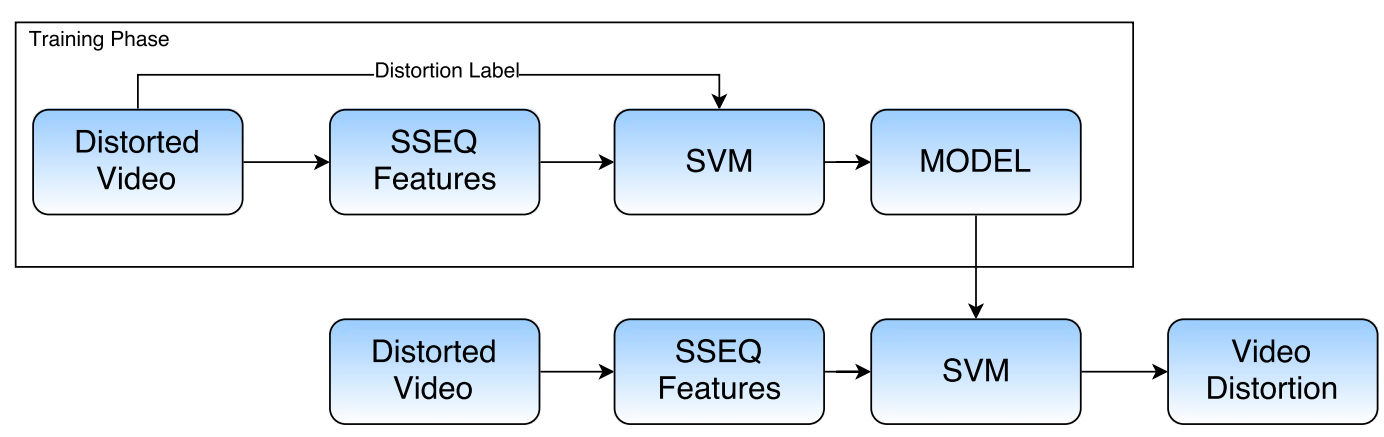

Figure 4.6: High-level overview of SSEQ algorithm for traning and testing stages.

Table 4.2: F1 score of the proposed SSEQ-based classifier for different temporal resolutions.

\begin{tabular}{|l|l|l|l|l|l|}
\cline { 2 - 6 } \multicolumn{1}{c|}{} & \multicolumn{5}{c|}{ FPS } \\
\cline { 2 - 6 } \multicolumn{1}{c|}{} & Original & 10 & 8 & 4 & 2 \\
\hline F1 Score & 0.7999 & 0.8021 & 0.8016 & 0.8043 & 0.7981 \\
\hline
\end{tabular}

We tested the proposed classifier using all videos from LIVE, CSIQ, IVPL and MCL-V databases. We divided these videos into training and testing sets. The training set consists of $80 \%$ of all videos, while the testing set consist of $20 \%$ of all videos. There are no content overlapping in the sets, i.e. videos corresponding to the same original were always in the same set. To compute the computational time of the SSEQ features extraction stage, we test the SSEQ video classifier with different frame rates, as shown in Table 4.2. We use the F1-Score to measure the accuracy performance of the classifier. We notice that even if we reduce the video temporal resolution to 2 FPS, the performance of the proposed SSEQ-based classifier is practically unaltered.

Table 4.3 shows the mean confusion matrix for 1,000 simulations of the proposed classifier, when computed for 2 FPS videos. The confusion matrix shows the proportion of positive and negative results of the video classifier (see Appendix I). These results show that the classifier can correctly identify videos distorted by MJPEG compression and white noise. But, it struggles to identify videos distorted by packet-loss. We believe that packet-loss is a complicated distortion to identify because it is, generally, a spatially and temporally localized distortion that does not 
affect all frames in the same way.

Table 4.4 shows the median of precision (the F1 score) over 1,000 simulations of the proposed SSEQ-based classifier. In more than half of these simulations, the proposed classifier identifies all videos distorted by white noise and MJPEG compression, but only $60 \%$ of the videos distorted by packet-loss. Also, from all videos classified as distorted by packet-loss, only $37 \%$ are actually videos distorted by packet-loss. Although this classifier cannot identify packet-loss, we will show in the next chapter that its performance is satisfactory for our propose.

Table 4.3: Mean Confusion Matrix of proposed SSEQ-based Distortion Classifier, for 1,000 simulations.

\begin{tabular}{|c|c|c|c|c|c|}
\hline & \multicolumn{4}{|c|}{ Distortion } \\
\hline & & Others & MJPEG & White Noise & packet-loss \\
\hline \multirow{4}{*}{ 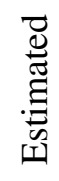 } & Others & 0.8891 & 0.0149 & 0.000 & 0.0960 \\
\hline & MJPEG & 0.0622 & 0.9378 & 0.0000 & 0.0000 \\
\hline & WhiteNoise & 0.0000 & 0.0000 & 0.9935 & 0.0065 \\
\hline & packet-loss & 0.6031 & 0.0005 & 0.0050 & 0.3914 \\
\hline
\end{tabular}

Table 4.4: Evaluation of the proposed SSEQ-based Distortion Classifier: median values of Precision, Recall, and F1 Score for 1,000 simulations.

\begin{tabular}{|l|l|l|l|}
\hline Distortion & Precision & Recall & F1 Score \\
\hline Others & 0.900 & 0.790 & \\
MJPEG & 1.000 & 1.000 & 0.799 \\
White Noise & 1.000 & 1.000 & \\
packet-loss & 0.375 & 0.600 & \\
\hline
\end{tabular}

\subsection{ADJUSTED PREDICTED SCORE}

One of our goals is to test the performance of video quality assessment methods for which the spatial resolution of the input videos is reduced. In this work, we noticed that all metrics are sensitive to video resolution reduction. When the spatial resolution is reduced, the video quality prediction is lower than the quality prediction obtained for a video in the original resolution. For example, Figure 4.7 shows ViS3's predicted scores for the CSIQ database, for the original spatial resolution $(768 \times 432)$ and for a reduced verison $(384 \times 216)$. Notice that, for videos in the reduced $384 \times 216$ resolution, ViS3's predicted scores vary from $10^{-2}$ to 10 . But, for the same videos in $768 \times 432$ resolution, they vary from $10^{-1}$ to 10 . So, when we evaluate quality using a reduced version of the video, the quality prediction value tends to be lower than the score obtained for the original resolution.

In this work, we reduce the videos to $768 \times 432$ or $384 \times 216$, depending on the video distortion. The $768 \times 432$ resolution was chosen because this is a resolution commonly used in the databases, like for example LIVE. The LIVE database has the lowest spatial resolution among the databases used in this work. In order for the predicted scores have the same range, independent of the video resolution, we use a logistic function to normalize the predicted scores. Figure 4.8 shows an 


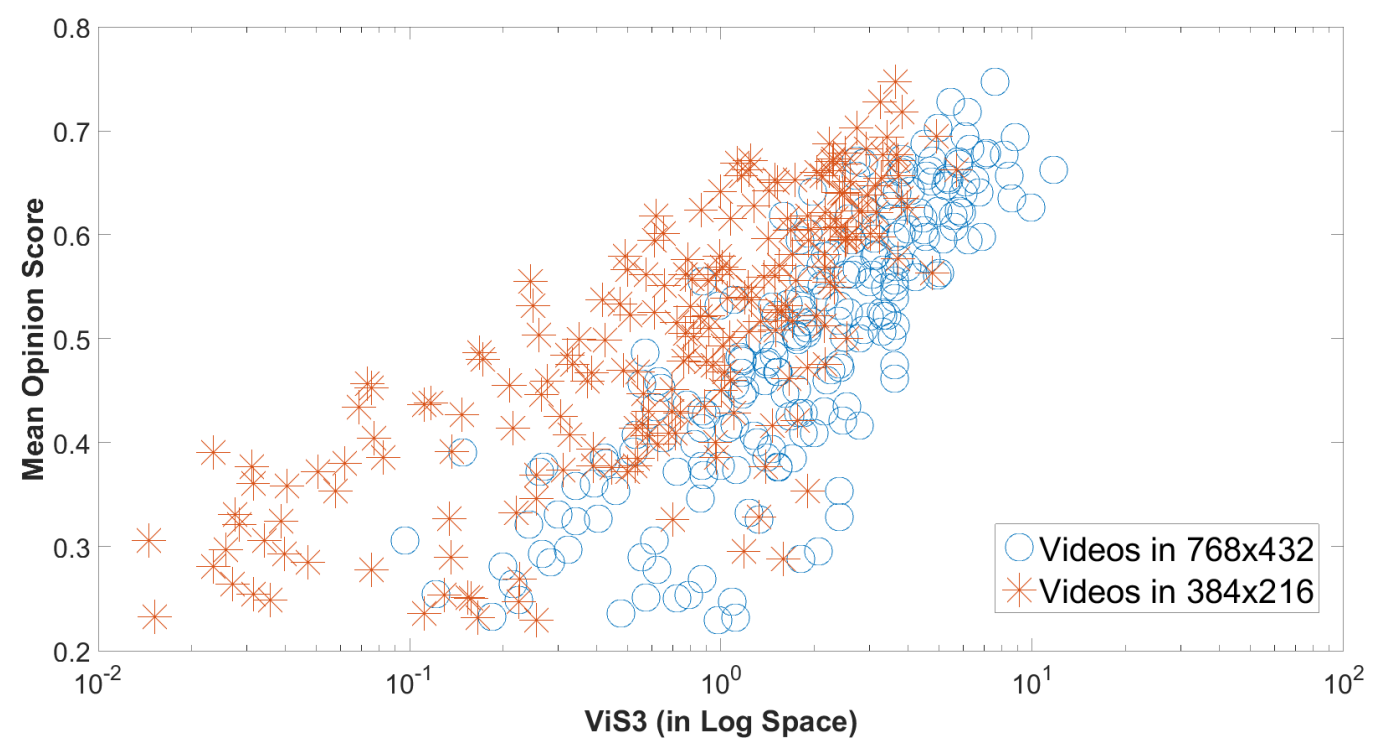

Figure 4.7: Plot of ViS3 versus Mean Opnion Score. The videos used were from CSIQ Database and they were downsampling to $768 \times 432$ and $384 \times 216$.

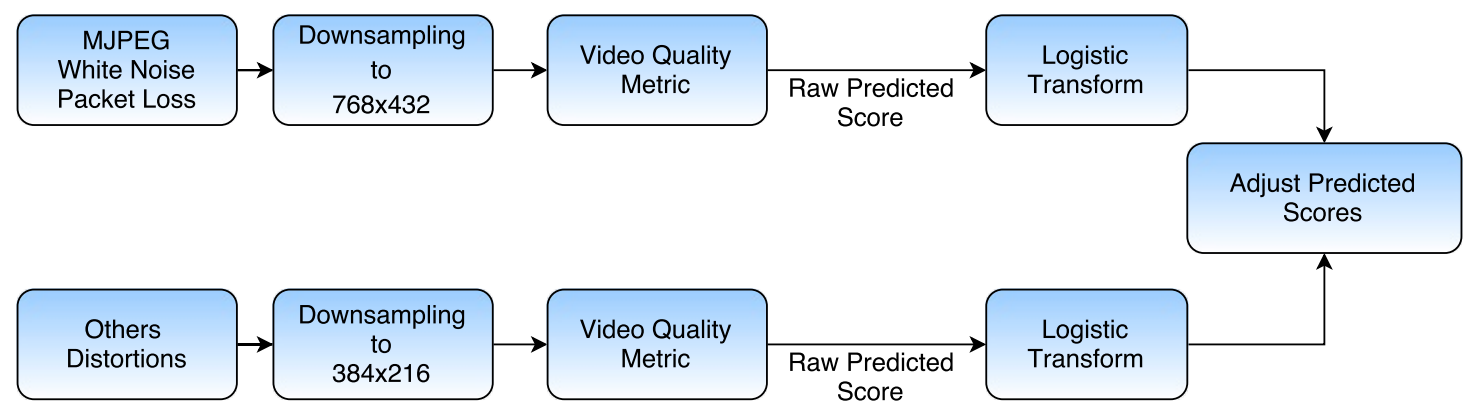

Figure 4.8: High Level Overview of the Adjustment of Predicted Score.

overview operations performed to adjust the predicted score. As mentioned earlier, the downsampling operation is performed using a bicubic interpolation in each frame [58], which generally gives better results than simpler interpolation algorithms like nearest neighbor and bilinear. Also, bicubic interpolation is the standard used in softwares like ffmpeg, Matlab, and Adobe Photoshop [20].

We chose the logistic function to normalize the scores from different resolutions because it is the scaling transform recommended by the Video Quality Experts Group (VQEG) to remove non-linearity behaviors and facilitate the comparison among scores provided by different video quality assessment methods [59]. The logistic function is given by the following equation:

$$
f(x)=\frac{\tau_{1}}{1+\tau_{2} \cdot \exp \left(-\tau_{3} \cdot x\right)},
$$

where $x$ is the predicted score and $\tau_{1}, \tau_{2}$ and $\tau_{3}$ are parameters used to provide the best fit of the predicted score to the subjective score. After using the logistic function, each score is in the same scale as the subjective scores and, therefore, we can compare them. 


\section{RESULTS}

As mentioned in previous chapters, one of the challenges for video quality assessment methods is the runtime performance $[12,13]$. These methods have become more complex over the last decades, with some of the algorithms being extremely slow. Reducing the spatial resolution of a video is one of the simplest and fastest methods to improve the running time of a video quality assessment method. However, reducing the video resolution can introduce new distortions and affect the quality prediction accuracy.

Based on the idea of reducing the spatial resolution, in this work we proposed a framework, which improves the running time performance of video quality assessment methods, without affecting its accuracy performance. In the previous chapters, we detailed this framework and proposed two video classifiers that can be used in the framework. In this chapter, we study which distortions are more sensitive to the video resolution reduction. We use the proposed classifiers to identify these videos, perform a video resolution reduction, and test the framework using both classifiers. Also, we compute the running time of the video quality assessment methods to compare it to the running times of the same method using the proposed framework.

This chapter is divided in two sections. In the first section, we describe the experimental setup used in our experiments. In the second section, we discuss our experimental results.

\subsection{EXPERIMENTAL SETUP}

The experiments were performed on an Intel i7-4790 processor at 3.60GHz. For all video quality assessment methods, except for the SSTS-GMSD, the Matlab code was provided by the authors. To evaluate the performance of each algorithm, we chose four video quality databases, which have different resolutions and distortions. A description of the specifications of each database was given in Chapter 3. For comparison purposes, Table 5.1 shows a summary of these specifications. Notice that the databases are very different form each other. For example, the videos of the IVP and MCL-V databases have a higher resolution than the videos in the LIVE and CSIQ databases. On the other hand, CSIQ has more types of distortions and different temporal resolutions than the other databases. It is worth pointing out that in the 'real world' videos do not have the same resolution or are degraded by the same distortions. Therefore, it is important to that the quality databases used in the test have a high content, resolution, format, and distortion diversity. 
Table 5.1: Comparison of Video Quality Databases Specifications.

\begin{tabular}{|l|c|c|c|c|c|}
\cline { 2 - 6 } \multicolumn{1}{c|}{} & $\begin{array}{c}\text { Reference } \\
\text { Videos }\end{array}$ & $\begin{array}{c}\text { Total } \\
\text { Videos }\end{array}$ & $\begin{array}{c}\text { Spatial } \\
\text { Resolution }\end{array}$ & $\begin{array}{c}\text { Temporal } \\
\text { Resolution }\end{array}$ & Type of Distortions \\
\hline LIVE & 10 & 150 & $768 \times 432$ & 25,50 & $\begin{array}{c}\text { MPEG-2, H.264 } \\
\text { Packet-Loss }\end{array}$ \\
\hline CSIQ & 12 & 216 & $832 \times 480$ & $\begin{array}{c}24,25,30 \\
50,60\end{array}$ & $\begin{array}{c}\text { H.264, H.265, Wavelet, } \\
\text { MJPEG, White Noise, } \\
\text { Packet-Loss }\end{array}$ \\
\hline IVP & 10 & 128 & $1920 \times 1088$ & 25 & $\begin{array}{c}\text { MPEG-2, H.264, } \\
\text { Wavelet, Packet-Loss }\end{array}$ \\
\hline MCL-V & 12 & 96 & $1920 \times 1080$ & $24,25,30$ & H.264, Upscaling \\
\hline
\end{tabular}

\subsection{EXPERIMENTAL RESULTS}

In this section, we present all the tests performed in this work. First, we study the effect that reducing the video spatial resolution has on the accuracy performance of video quality assessment methods. Then, we study how different distortions affect the quality assessment methods. From these results, we obtain the necessary information to design the proposed framework. Next, we present the results of the proposed framework using two different video classifiers. Finally, we present additional tests on the effect that reducing the temporal resolution has on the performance of video quality assessment methods.

To study how the video resolution can affect the method accuracy performance, we used different video quality assessment methods to estimate the quality of videos with different spatial resolutions. To evaluate the prediction accuracy of each video quality assessment method, we calculate the SCC between the subjective scores provided by the databases and the objectives scores predicted by these methods [59]. The absolute value of SCC varies from 1 (best) to 0 (worst). When the SCC is closer to \pm 1 , the predicted scores given by the quality assessment methods are similar to the subjective scores provided by the database (see Appendix II).

It is worth mentioning again that, for some video quality assessment methods, reducing the video resolution can cause a decrease in prediction quality accuracy. Tables 5.2- 5.5 shows the SCC values for a set of video quality assessment methods, which were tested on the LIVE, CSIQ, IVP, and MCL-V databases, respectively. Notice that for the IVP and MCL-V databases, there is an improvement on accuracy performance when the video resolution is reduced, but for LIVE and CSIQ databases the accuracy performance decreases. The accuracy performance of PSNR, SSIM and GMSD increases for the LIVE database, but only the accuracy performance of SSIM increases for the CSIQ Database. It is worth pointing out that the CSIQ Database has more distortions than the others databases, while MCL-V and IVP databases have videos with a higher resolution (1920x1080 for MCL-V and 1920x1088 for IVP).

In summary, the accuracy performance of the methods vary according to the databases, i.e. according to the content and type of distortions. So, to verify if the video distortion has influence on the accuracy performance of the methods, we analyze the data in groups separated by the types 
Table 5.2: Spearman Correlation Coefficient (SCC) of the quality assessment methods, tested on the LIVE database.

\begin{tabular}{|l|l|l|l|l|}
\hline & $768 \times 432$ & $640 \times 360$ & $480 \times 270$ & $384 \times 216$ \\
\hline ViS3 & $\mathbf{0 . 8 1 6 8}$ & 0.7995 & 0.7786 & 0.7595 \\
\hline STRRED & $\mathbf{0 . 8 0 0 7}$ & 0.7902 & 0.7691 & 0.7570 \\
\hline SSTS-GMSD & $\mathbf{0 . 8 3 8 9}$ & 0.8255 & 0.7988 & 0.7777 \\
\hline GMSD & 0.7262 & 0.7354 & 0.7348 & 0.7264 \\
\hline SSIM & 0.5251 & 0.6131 & 0.6696 & $\mathbf{0 . 7 0 0 2}$ \\
\hline PSNR & 0.5233 & 0.5750 & 0.6084 & $\mathbf{0 . 6 2 8 5}$ \\
\hline
\end{tabular}

Table 5.3: Spearman Correlation Coefficient (SCC) of the quality assessment methods, tested on the CSIQ database.

\begin{tabular}{|l|l|l|l|l|}
\hline & $768 \times 432$ & $640 \times 360$ & $480 \times 270$ & $384 \times 216$ \\
\hline ViS3 & $\mathbf{0 . 8 5 8 1}$ & 0.8574 & 0.8271 & 0.7897 \\
\hline STRRED & $\mathbf{0 . 8 0 3 5}$ & 0.7881 & 0.7666 & 0.7424 \\
\hline SSTS-GMSD & $\mathbf{0 . 8 4 5 7}$ & 0.8411 & 0.7950 & 0.7458 \\
\hline GMSD & $\mathbf{0 . 8 4 4 9}$ & $\mathbf{0 . 8 4 4 9}$ & 0.8107 & 0.7729 \\
\hline SSIM & 0.6236 & 0.6566 & 0.7016 & $\mathbf{0 . 7 2 4 1}$ \\
\hline PSNR & $\mathbf{0 . 5 8 9 6}$ & 0.5853 & 0.5696 & 0.5585 \\
\hline
\end{tabular}

Table 5.4: Spearman Correlation Coefficient (SCC) of the quality assessment methods, tested on the IVP database.

\begin{tabular}{|l|l|l|l|l|l|l|l|}
\hline & $1920 \times 1080$ & $1280 \times 720$ & $960 \times 540$ & $768 \times 432$ & $640 \times 360$ & $480 \times 270$ & $384 \times 216$ \\
\hline ViS3 & 0.8023 & 0.8822 & 0.8995 & 0.8968 & $\mathbf{0 . 9 0 9 4}$ & 0.8981 & 0.8912 \\
\hline STRRED & 0.7378 & $\mathbf{0 . 7 4 2 6}$ & 0.7308 & 0.7296 & 0.7177 & 0.7160 & 0.7144 \\
\hline SSTS-GMSD & 0.7560 & 0.8236 & 0.8683 & 0.8830 & $\mathbf{0 . 8 8 8 0}$ & $\mathbf{0 . 8 8 8 0}$ & 0.8722 \\
\hline GMSD & 0.6924 & 0.7973 & 0.8493 & 0.8671 & 0.8791 & $\mathbf{0 . 8 8 3 3}$ & 0.8672 \\
\hline SSIM & 0.3739 & 0.4749 & 0.5277 & 0.5677 & 0.6077 & 0.6629 & $\mathbf{0 . 6 9 6 5}$ \\
\hline PSNR & 0.6566 & 0.7050 & 0.7312 & 0.7707 & 0.8086 & 0.8333 & $\mathbf{0 . 8 3 7 4}$ \\
\hline
\end{tabular}

Table 5.5: Spearman Correlation Coefficient (SCC) of the quality assessment methods, tested on the MCL-V database.

\begin{tabular}{|l|l|l|l|l|l|l|l|}
\hline & $1920 \times 1080$ & $1280 \times 720$ & $960 \times 540$ & $768 \times 432$ & $640 \times 360$ & $480 \times 270$ & $384 \times 216$ \\
\hline ViS3 & 0.6361 & 0.6902 & 0.7167 & 0.7307 & 0.7408 & 0.7516 & $\mathbf{0 . 7 6 7 4}$ \\
\hline STRRED & 0.7433 & 0.7433 & 0.7738 & 0.7938 & 0.7964 & 0.8035 & $\mathbf{0 . 8 1 7 7}$ \\
\hline SSTS-GMSD & 0.6855 & 0.7395 & 0.7575 & 0.7768 & 0.7850 & 0.7989 & $\mathbf{0 . 8 0 0 0}$ \\
\hline GMSD & 0.6449 & 0.7068 & 0.7234 & 0.7369 & 0.7483 & 0.7625 & $\mathbf{0 . 7 7 0 8}$ \\
\hline SSIM & 0.4018 & 0.5408 & 0.6062 & 0.6415 & 0.6632 & 0.6961 & $\mathbf{0 . 7 1 0 5}$ \\
\hline PSNR & 0.4640 & 0.5328 & 0.5791 & 0.6097 & 0.6347 & 0.6668 & $\mathbf{0 . 6 8 7 6}$ \\
\hline
\end{tabular}

of distortion. These results are presented in Tables 5.6- 5.9.

Table 5.6 shows the accuracy performance of each method when the video resolution is reduced, tested on the CSIQ Database. The accuracy performance of PSNR and SSIM improves when the video resolution is reduced. For PSNR, the improvement is $25.47 \%$ for videos compressed with H.264, 33.30\% for videos compressed with MPEG2, and 12.49\% for videos distorted by packet-loss. For SSIM, the improvement is $4.23 \%$ for videos compressed with H.264, $21.37 \%$ for videos compressed with MPEG2, and $32.88 \%$ for videos distorted by packet-loss. For GMSD, the accuracy performance improves for videos distorted by packet-loss (4.59\% of improvement), 
Table 5.6: Spearman Correlation Coefficient (SCC) of the quality assessment methods, tested on the LIVE database.

\begin{tabular}{|l|l|l|l|l|l|}
\hline \multirow{3}{*}{ Metric } & & \multicolumn{4}{|c|}{ Video Resolution } \\
\cline { 2 - 6 } ViS3 & Distortion & $768 \times 432$ & $640 \times 360$ & $480 \times 270$ & $384 \times 216$ \\
\hline \multirow{5}{*}{ STRRED } & H.264 & $\mathbf{0 . 7 6 8 5}$ & 0.7598 & 0.7371 & 0.7405 \\
& MPEG2 & 0.7362 & 0.7639 & $\mathbf{0 . 7 8 0 6}$ & 0.7774 \\
& Packet-Loss & $\mathbf{0 . 8 3 7 2}$ & 0.8298 & 0.8195 & 0.8270 \\
\hline \multirow{3}{*}{ SSTS-GMSD } & H.264 & 0.8193 & 0.8223 & 0.8113 & $\mathbf{0 . 8 3 0 4}$ \\
& MPEG2 & $\mathbf{0 . 7 1 9 3}$ & 0.6986 & 0.6703 & 0.6769 \\
& Packet-Loss & $\mathbf{0 . 7 9 3 4}$ & 0.7904 & 0.7693 & 0.7510 \\
\hline \multirow{5}{*}{ GMSD } & H.264 & $\mathbf{0 . 7 9 7 4}$ & 0.7788 & 0.7615 & 0.7623 \\
& MPEG2 & 0.8125 & 0.8121 & 0.8362 & $\mathbf{0 . 8 4 7 0}$ \\
& Packet-Loss & $\mathbf{0 . 8 1 5 1}$ & 0.8148 & 0.8044 & 0.7963 \\
\hline \multirow{3}{*}{ SSIM } & H.264 & $\mathbf{0 . 6 4 7 1}$ & 0.6452 & 0.6302 & 0.6349 \\
& MPEG2 & $\mathbf{0 . 6 9 1 5}$ & 0.6836 & 0.6725 & 0.6778 \\
& Packet-Loss & 0.7457 & 0.7626 & 0.7775 & $\mathbf{0 . 7 8 0 0}$ \\
\hline PSNR & H.264 & 0.6561 & $\mathbf{0 . 7 1 7 1}$ & 0.6961 & 0.6839 \\
& MPEG2 & 0.5609 & 0.6112 & 0.6679 & $\mathbf{0 . 6 8 0 8}$ \\
& Packet-Loss & 0.5151 & 0.5716 & 0.6337 & $\mathbf{0 . 6 8 4 5}$ \\
\hline & H.264 & 0.4730 & 0.5424 & 0.5856 & $\mathbf{0 . 5 9 3 4}$ \\
\cline { 2 - 6 } & MPEG2 & 0.3830 & 0.4510 & 0.4825 & $\mathbf{0 . 5 1 0 6}$ \\
& Packet-Loss & 0.5799 & 0.6294 & $\mathbf{0 . 6 5 5 0}$ & 0.6523 \\
\hline
\end{tabular}

but decreases by $1.88 \%$ and $2.14 \%$ for videos compressed with H.264 and MPEG2, respectively. For SSTS-GMSD, the accuracy performance improves by $4.25 \%$ for videos compressed with MPEG2, but decreases for videos compressed by H.264 and distorted with packet-loss by $4.40 \%$ and $2.30 \%$, respectively. For STRRED, the accuracy performance improves by $1.35 \%$ for videos compressed by H.264, decreases by $5.89 \%$ for videos compressed with MPEG2, and by 5.35\% for videos distorted with packet-loss. Finally, for ViS3, the accuracy performance improves by $5.61 \%$ for videos compressed with MPEG2, decreases by $3.64 \%$ for videos compressed with H.264 and by $1.22 \%$ for videos distorted with packet-loss.

Table 5.7 shows the accuracy performance of each method when the video resolution is reduced, for the CSIQ Database. Notice that the accuracy performances of ViS3, SSTS-GMSD, SSIM, and PSNR increase when the resolution is reduced, for videos compressed by H.264, H.265, and Wavelet. The gain for videos distorted by H.264 Compression is $0.58 \%$ for ViS3, $1.42 \%$ for SSTS-GMSD, $6.74 \%$ for SSIM and $7.08 \%$ for PSNR. The gain for videos distorted by H.265 Compression is $1.24 \%$ for ViS3, $0.06 \%$ for SSTS-GMSD, 9.13\% for SSIM and 5.60\% for PSNR. The gain for videos distorted by Wavelet Compression is $2.89 \%$ for ViS3, $1.88 \%$ for SSTS-GMSD, 5.30\% for SSIM and 7.78\% for PSNR. The accuracy performance of all methods decreases for videos distorted by MJPEG compression, packet-loss, and white noise. For all methods, the worst accuracy performance corresponds to the resolution $384 \times 216$ for videos compressed by MJPEG. The decrease in accuracy performance for videos compressed with MJPEG is $26.08 \%$ for ViS3, $89.51 \%$ for STRRED, 9.46\% for SSTS-GMSD, 16.54\% for GMSD, 6.89\% for SSIM, and $63.75 \%$ for PSNR. 
Table 5.7: Spearman Correlation Coefficient (SCC) of the quality assessment methods, tested on the CSIQ database.

\begin{tabular}{|c|c|c|c|c|c|}
\hline \multirow[b]{2}{*}{ Metric } & \multirow[b]{2}{*}{ Distortion } & \multicolumn{4}{|c|}{ Video Resolution } \\
\hline & & $768 \times 432$ & $640 \times 360$ & $480 \times 270$ & $384 \times 216$ \\
\hline \multirow[t]{6}{*}{ ViS3 } & H.264 & 0.9300 & 0.9400 & 0.9421 & 0.9354 \\
\hline & Н.265 & 0.9331 & 0.9413 & 0.9537 & 0.9447 \\
\hline & MJPEG & 0.7776 & 0.7817 & 0.6829 & 0.5748 \\
\hline & Packet-Loss & 0.8381 & 0.8319 & 0.8051 & 0.7907 \\
\hline & Wavelet & 0.9161 & 0.9238 & 0.9398 & 0.9426 \\
\hline & White Noise & 0.9210 & 0.9192 & 0.9081 & 0.8929 \\
\hline \multirow[t]{6}{*}{ STRRED } & H.264 & 0.9768 & 0.9753 & 0.9686 & 0.9637 \\
\hline & H.265 & 0.9112 & 0.9238 & 0.9230 & 0.9202 \\
\hline & MJPEG & 0.5964 & 0.4839 & 0.1611 & 0.0625 \\
\hline & Packet-Loss & 0.8468 & 0.8551 & 0.8435 & 0.8353 \\
\hline & Wavelet & 0.9457 & 0.9351 & 0.9290 & 0.9266 \\
\hline & White Noise & 0.9192 & 0.9079 & 0.8772 & 0.8528 \\
\hline \multirow[t]{6}{*}{ SSTS-GMSD } & Н.264 & 0.9277 & 0.9292 & 0.9318 & 0.9282 \\
\hline & H.265 & 0.9454 & 0.9552 & 0.9480 & 0.9472 \\
\hline & MJPEG & 0.8893 & 0.8885 & 0.8528 & 0.7840 \\
\hline & Packet-Loss & 0.8108 & 0.8031 & 0.7743 & 0.7495 \\
\hline & Wavelet & 0.8705 & 0.8718 & 0.8754 & 0.8754 \\
\hline & White Noise & 0.8847 & 0.8708 & 0.8072 & 0.7529 \\
\hline \multirow[t]{6}{*}{ GMSD } & H.264 & 0.9441 & 0.9503 & 0.9532 & 0.9503 \\
\hline & Н.265 & 0.9408 & 0.9439 & 0.9326 & 0.9279 \\
\hline & MJPEG & 0.8994 & 0.9192 & 0.8741 & 0.7586 \\
\hline & Packet-Loss & 0.8631 & 0.8577 & 0.8512 & 0.8111 \\
\hline & Wavelet & 0.8764 & 0.8762 & 0.8623 & 0.8610 \\
\hline & White Noise & 0.9094 & 0.9068 & 0.8808 & 0.8391 \\
\hline \multirow[t]{6}{*}{ SSIM } & H.264 & 0.8903 & 0.9143 & 0.9485 & 0.9503 \\
\hline & Н.265 & 0.8654 & 0.9053 & 0.9290 & 0.9444 \\
\hline & MJPEG & 0.8373 & 0.8456 & 0.8041 & 0.7797 \\
\hline & Packet-Loss & 0.8556 & 0.8535 & 0.8517 & 0.8492 \\
\hline & Wavelet & 0.8154 & 0.8317 & 0.8597 & 0.8587 \\
\hline & White Noise & 0.9269 & 0.9292 & 0.9223 & 0.9122 \\
\hline \multirow[t]{6}{*}{ PSNR } & H.264 & 0.8687 & 0.8914 & 0.9218 & 0.9302 \\
\hline & H.265 & 0.8556 & 0.8782 & 0.8986 & 0.9035 \\
\hline & MJPEG & 0.4672 & 0.4296 & 0.2847 & 0.1694 \\
\hline & Packet-Loss & 0.8481 & 0.8409 & 0.8322 & 0.8245 \\
\hline & Wavelet & 0.7941 & 0.8265 & 0.8479 & 0.8559 \\
\hline & White Noise & 0.9048 & 0.9060 & 0.9079 & 0.8947 \\
\hline
\end{tabular}

Table 5.8 shows the accuracy performance for the IVP Database. Notice that, for most spatial resolutions, the accuracy performance of videos distorted by packet-loss decreases for ViS3, STRRED, and SSTS-GMSD. Nevertheless, it improves for the 768x432 resolution. For GMSD, the best accuracy performance is obtained for videos distorted by packet-loss, in a 640x360 resolution. The accuracy performance for videos compressed with Wavelet and H.264 improves for almost all methods, with the exception of STRRED for videos compressed with H.264.

Table 5.9 shows the accuracy performance for each method when the video resolution is re- 
Table 5.8: Spearman Correlation Coefficient (SCC) of the quality assessment methods, tested on the IVP database.

\begin{tabular}{|c|c|c|c|c|c|c|c|c|}
\hline \multirow[b]{2}{*}{ Metric } & \multirow[b]{2}{*}{ Distortion } & \multicolumn{7}{|c|}{ Video Resolution } \\
\hline & & $1920 \times 1080$ & $1280 \times 720$ & $960 \times 540$ & $768 \times 432$ & $640 \times 360$ & $480 \times 270$ & $384 \times 216$ \\
\hline \multirow[t]{4}{*}{ ViS3 } & Wavelet & 0.9186 & 0.9212 & 0.9132 & 0.9253 & 0.9368 & 0.9212 & 0.9306 \\
\hline & H.264 & 0.8477 & 0.8735 & 0.8681 & 0.8538 & 0.8787 & 0.8864 & 0.8906 \\
\hline & MPEG2 & 0.7918 & 0.8274 & 0.8527 & 0.8509 & 0.8394 & 0.8558 & 0.8658 \\
\hline & Packet-Loss & 0.7504 & 0.8057 & 0.7947 & 0.7750 & 0.7526 & 0.7280 & 0.6962 \\
\hline \multirow[t]{4}{*}{ STRRED } & Wavelet & 0.8554 & 0.8839 & 0.8932 & 0.8914 & 0.8910 & 0.8870 & 0.8843 \\
\hline & H.264 & 0.8614 & 0.8505 & 0.8493 & 0.8477 & 0.8435 & 0.8454 & 0.8458 \\
\hline & MPEG2 & 0.6752 & 0.6400 & 0.6427 & 0.6400 & 0.6360 & 0.6489 & 0.6570 \\
\hline & Packet-Loss & 0.6650 & 0.7028 & 0.6765 & 0.6661 & 0.6650 & 0.6158 & 0.5977 \\
\hline \multirow{4}{*}{$\begin{array}{l}\text { SSTS- } \\
\text { GMSD }\end{array}$} & Wavelet & 0.8216 & 0.8509 & 0.8710 & 0.8763 & 0.8812 & 0.8968 & 0.9075 \\
\hline & H.264 & 0.8463 & 0.8587 & 0.8651 & 0.8726 & 0.8809 & 0.8841 & 0.8876 \\
\hline & MPEG2 & 0.7824 & 0.8073 & 0.8318 & 0.8443 & 0.8501 & 0.8714 & 0.8812 \\
\hline & Packet-Loss & 0.7663 & 0.7871 & 0.7937 & 0.7750 & 0.7603 & 0.7154 & 0.6907 \\
\hline \multirow[t]{4}{*}{ GMSD } & Wavelet & 0.8229 & 0.8763 & 0.8941 & 0.8790 & 0.9008 & 0.9110 & 0.9101 \\
\hline & H.264 & 0.8630 & 0.8780 & 0.8826 & 0.8856 & 0.8880 & 0.8897 & 0.8902 \\
\hline & MPEG2 & 0.8283 & 0.8256 & 0.8220 & 0.8211 & 0.8291 & 0.8251 & 0.8283 \\
\hline & Packet-Loss & 0.7121 & 0.8150 & 0.8413 & 0.8407 & 0.8467 & 0.8128 & 0.7849 \\
\hline \multirow[t]{4}{*}{ SSIM } & Wavelet & 0.7846 & 0.8029 & 0.8060 & 0.8140 & 0.8287 & 0.8492 & 0.8407 \\
\hline & H.264 & 0.6636 & 0.7752 & 0.8146 & 0.8310 & 0.8407 & 0.8462 & 0.8418 \\
\hline & MPEG2 & 0.5884 & 0.6436 & 0.6574 & 0.6783 & 0.6836 & 0.6690 & 0.6529 \\
\hline & Packet-Loss & 0.0482 & 0.1910 & 0.2638 & 0.3136 & 0.3985 & 0.4729 & 0.5161 \\
\hline \multirow[t]{4}{*}{ PSNR } & Wavelet & 0.8598 & 0.8603 & 0.8594 & 0.8759 & 0.8723 & 0.8914 & 0.8905 \\
\hline & Н.264 & 0.8218 & 0.8488 & 0.8681 & 0.8765 & 0.8811 & 0.8901 & 0.8901 \\
\hline & MPEG2 & 0.6948 & 0.7130 & 0.7384 & 0.7620 & 0.7539 & 0.7673 & 0.7749 \\
\hline & Packet-Loss & 0.6393 & 0.6793 & 0.7115 & 0.7340 & 0.7630 & 0.7822 & 0.8051 \\
\hline
\end{tabular}

Table 5.9: Spearman Correlation Coefficient (SCC) of the quality assessment methods, tested on the MCL-V database.

\begin{tabular}{|l|l|c|c|c|c|c|c|c|}
\hline \multirow{2}{*}{ Metric } & Distortion & $1920 \times 1080$ & $1280 \times 720$ & $960 \times 540$ & $768 \times 432$ & $640 \times 360$ & $480 \times 270$ & $384 \times 216$ \\
\hline ViS3 & H.264 & 0.5868 & 0.6625 & 0.6960 & 0.7273 & 0.7445 & 0.7655 & $\mathbf{0 . 7 7 8 0}$ \\
& Upscaling & 0.6890 & 0.7043 & 0.7245 & 0.7283 & 0.7304 & 0.7333 & $\mathbf{0 . 7 4 4 0}$ \\
\hline STRRED & H.264 & 0.7716 & 0.7716 & 0.7807 & 0.8055 & 0.8010 & 0.8035 & $\mathbf{0 . 8 1 3 9}$ \\
& Upscaling & 0.7040 & 0.7040 & 0.7436 & 0.7732 & 0.7714 & 0.7858 & $\mathbf{0 . 8 1 2 3}$ \\
\hline SSTS- & H.264 & 0.6921 & 0.7189 & 0.7423 & 0.7769 & 0.7867 & 0.7950 & $\mathbf{0 . 8 0 5 8}$ \\
GMSD & Upscaling & 0.6806 & 0.7477 & 0.7629 & 0.7785 & 0.7778 & 0.7843 & $\mathbf{0 . 7 9 6 0}$ \\
\hline GMSD & H.264 & 0.6452 & 0.7057 & 0.7332 & 0.7484 & 0.7635 & 0.7685 & $\mathbf{0 . 7 9 0 5}$ \\
& Upscaling & 0.6376 & 0.6789 & 0.6898 & 0.7021 & 0.6988 & 0.7100 & $\mathbf{0 . 7 2 9 2}$ \\
\hline SSIM & H.264 & 0.3545 & 0.5375 & 0.6172 & 0.6533 & 0.6764 & 0.7222 & $\mathbf{0 . 7 3 6 0}$ \\
& Upscaling & 0.4400 & 0.5300 & 0.5931 & 0.6242 & 0.6408 & 0.6616 & $\mathbf{0 . 6 7 5 4}$ \\
\hline PSNR & H.264 & 0.4215 & 0.5115 & 0.5634 & 0.5872 & 0.6203 & 0.6651 & $\mathbf{0 . 6 7 6 1}$ \\
& Upscaling & 0.4925 & 0.5382 & 0.5851 & 0.6216 & 0.6402 & 0.6537 & $\mathbf{0 . 6 8 3 4}$ \\
\hline
\end{tabular}

duced for the MCL-V Database. In this database, the accuracy performance of all methods are improved when the video resolution is reduced. For videos compressed with H.264, the improvement is $32.58 \%$ for ViS3, $5.48 \%$ for STRRED, $16.43 \%$ for SSTS-GMSD, 22.52\% for GMSD, 
Table 5.10: Spearman Correlation Coefficient (SCC) of the VIS3 metric, tested on all databases.

\begin{tabular}{|l|l|l|l|l|}
\hline \multirow{2}{*}{ Distortion } & \multicolumn{4}{|c|}{ Video Resolution } \\
\cline { 2 - 5 } & $768 \times 432$ & $640 \times 360$ & $480 \times 270$ & $384 \times 216$ \\
\hline Wavelet & 0.8864 & 0.8988 & 0.9093 & $\mathbf{0 . 9 1 2 5}$ \\
\hline H.264 & 0.8354 & 0.8469 & $\mathbf{0 . 8 4 7 9}$ & 0.8473 \\
\hline H.265 & 0.9331 & 0.9413 & $\mathbf{0 . 9 5 3 7}$ & 0.9447 \\
\hline MJPEG & 0.7776 & $\mathbf{0 . 7 8 1 7}$ & 0.6829 & 0.5748 \\
\hline MPEG2 & 0.8561 & 0.8766 & $\mathbf{0 . 8 8 5 1}$ & 0.8848 \\
\hline Upscaling & 0.7283 & 0.7304 & 0.7333 & $\mathbf{0 . 7 4 4 0}$ \\
\hline Packet-Loss & $\mathbf{0 . 8 1 7 4}$ & 0.8179 & 0.8087 & 0.8028 \\
\hline White Noise & $\mathbf{0 . 9 2 1 0}$ & 0.9192 & 0.9081 & 0.8929 \\
\hline
\end{tabular}

Table 5.11: Spearman Correlation Coefficient (SCC) of the STRRED metric, tested on all databases.

\begin{tabular}{|l|l|l|l|l|}
\hline \multirow{2}{*}{ Distortion } & \multicolumn{4}{|c|}{ Video Resolution } \\
\cline { 2 - 5 } & $768 \times 432$ & $640 \times 360$ & $480 \times 270$ & $384 \times 216$ \\
\hline Wavelet & 0.8505 & 0.8499 & 0.8548 & $\mathbf{0 . 8 6 3 8}$ \\
\hline H.264 & 0.8822 & 0.8816 & 0.8810 & $\mathbf{0 . 8 8 5 9}$ \\
\hline H.265 & 0.9112 & $\mathbf{0 . 9 2 3 8}$ & 0.9230 & 0.9202 \\
\hline MJPEG & $\mathbf{0 . 5 9 6 4}$ & 0.4839 & 0.1611 & 0.0625 \\
\hline MPEG2 & $\mathbf{0 . 7 9 9 3}$ & 0.7891 & 0.7874 & 0.7935 \\
\hline Upscaling & 0.7732 & 0.7714 & 0.7858 & $\mathbf{0 . 8 1 2 3}$ \\
\hline Packet-Loss & 0.7881 & $\mathbf{0 . 7 9 0 6}$ & 0.7793 & 0.7672 \\
\hline White Noise & $\mathbf{0 . 9 1 9 2}$ & 0.9079 & 0.8772 & 0.8528 \\
\hline
\end{tabular}

Table 5.12: Spearman Correlation Coefficient (SCC) of the SSTS-GMSD metric, tested on all databases.

\begin{tabular}{|l|l|l|l|l|}
\hline \multirow{2}{*}{ Distortion } & \multicolumn{4}{|c|}{ Video Resolution } \\
\cline { 2 - 5 } & $768 \times 432$ & $640 \times 360$ & $480 \times 270$ & $384 \times 216$ \\
\hline Wavelet & 0.8035 & 0.8109 & 0.8234 & $\mathbf{0 . 8 3 6 7}$ \\
\hline H.264 & 0.8520 & 0.8520 & 0.8539 & $\mathbf{0 . 8 5 8 3}$ \\
\hline H.265 & 0.9454 & $\mathbf{0 . 9 5 5 2}$ & 0.9480 & 0.9472 \\
\hline MJPEG & $\mathbf{0 . 8 8 9 3}$ & 0.8885 & 0.8528 & 0.7840 \\
\hline MPEG2 & 0.8776 & 0.8905 & 0.9032 & $\mathbf{0 . 9 0 7 4}$ \\
\hline Upscaling & 0.7785 & 0.7778 & 0.7843 & $\mathbf{0 . 7 9 6 0}$ \\
\hline Packet-Loss & 0.7785 & $\mathbf{0 . 7 8 7 6}$ & 0.7794 & 0.7703 \\
\hline White Noise & $\mathbf{0 . 8 8 4 7}$ & 0.8708 & 0.8072 & 0.7529 \\
\hline
\end{tabular}

$107.62 \%$ for SSIM, and $60.40 \%$ for PSNR. For videos distorted by Upscaling, the improvement is $7.98 \%$ for ViS3, $15.38 \%$ for STRRED, $16.96 \%$ for SSTS-GMSD, $14.36 \%$ for GMSD, $53.50 \%$ for SSIM, and $38.76 \%$ for PSNR.

From these results, we can notice that, for all methods, the accuracy performance decreases when videos distorted by MJPEG compression and white noise have their spatial resolution reduced. For videos distorted by packet-loss, the accuracy performance of most methods is better when the video resolution is $640 \times 360$ or above.

To better evaluate the prediction quality accuracy of each method for each type of distortion, 
Table 5.13: Spearman Correlation Coefficient (SCC) of the GMSD metric, tested on all databases.

\begin{tabular}{|l|l|l|l|l|}
\hline \multirow{2}{*}{ Distortion } & \multicolumn{4}{|c|}{ Video Resolution } \\
\cline { 2 - 5 } & $768 \times 432$ & $640 \times 360$ & $480 \times 270$ & $384 \times 216$ \\
\hline Wavelet & 0.8430 & 0.8618 & 0.8655 & $\mathbf{0 . 8 6 9 8}$ \\
\hline H.264 & 0.8405 & 0.8439 & 0.8482 & $\mathbf{0 . 8 5 1 6}$ \\
\hline H.265 & 0.9408 & $\mathbf{0 . 9 4 3 9}$ & 0.9326 & 0.9279 \\
\hline MJPEG & 0.8994 & $\mathbf{0 . 9 1 8 1}$ & 0.8741 & 0.7506 \\
\hline MPEG2 & 0.8282 & 0.8326 & 0.8360 & $\mathbf{0 . 8 4 2 5}$ \\
\hline Upscaling & 0.7031 & 0.7057 & 0.7207 & $\mathbf{0 . 7 2 8 9}$ \\
\hline Packet-Loss & 0.7828 & 0.7941 & $\mathbf{0 . 7 9 7 9}$ & 0.7949 \\
\hline White Noise & $\mathbf{0 . 9 0 9 4}$ & 0.9068 & 0.8808 & 0.8347 \\
\hline
\end{tabular}

Table 5.14: Spearman Correlation Coefficient (SCC) of the SSIM metric, tested on all databases.

\begin{tabular}{|l|l|l|l|l|}
\hline \multirow{2}{*}{ Distortion } & \multicolumn{4}{|c|}{ Video Resolution } \\
\cline { 2 - 5 } & $768 \times 432$ & $640 \times 360$ & $480 \times 270$ & $384 \times 216$ \\
\hline Wavelet & 0.7981 & 0.8197 & 0.8469 & $\mathbf{0 . 8 4 9 7}$ \\
\hline H.264 & 0.7962 & 0.8170 & 0.8344 & $\mathbf{0 . 8 3 9 8}$ \\
\hline H.265 & 0.8654 & 0.9053 & 0.9290 & $\mathbf{0 . 9 4 4 4}$ \\
\hline MJPEG & 0.8373 & $\mathbf{0 . 8 4 5 6}$ & 0.8041 & 0.7797 \\
\hline MPEG2 & 0.7682 & 0.7954 & 0.8160 & $\mathbf{0 . 8 1 9 0}$ \\
\hline Upscaling & 0.6242 & 0.6408 & 0.6616 & $\mathbf{0 . 6 7 5 4}$ \\
\hline Packet-Loss & 0.5446 & 0.5732 & 0.6112 & $\mathbf{0 . 6 5 1 7}$ \\
\hline White Noise & 0.9269 & $\mathbf{0 . 9 2 9 2}$ & 0.9223 & 0.9122 \\
\hline
\end{tabular}

Table 5.15: Spearman Correlation Coefficient (SCC) of the PSNR metric, tested on all databases.

\begin{tabular}{|l|l|l|l|l|}
\hline \multirow{2}{*}{ Distortion } & \multicolumn{4}{|c|}{ Video Resolution } \\
\cline { 2 - 5 } & $768 \times 432$ & $640 \times 360$ & $480 \times 270$ & $384 \times 216$ \\
\hline Wavelet & 0.7756 & 0.8004 & 0.8184 & $\mathbf{0 . 8 2 9 3}$ \\
\hline H.264 & 0.7488 & 0.7743 & 0.7994 & $\mathbf{0 . 8 1 0 5}$ \\
\hline H.265 & 0.8556 & 0.8782 & 0.8986 & $\mathbf{0 . 9 0 3 5}$ \\
\hline MJPEG & $\mathbf{0 . 4 6 7 2}$ & 0.4296 & 0.2847 & 0.1694 \\
\hline MPEG2 & 0.7012 & 0.7289 & 0.7520 & $\mathbf{0 . 7 6 3 2}$ \\
\hline Upscaling & 0.6216 & 0.6402 & 0.6537 & $\mathbf{0 . 6 8 3 4}$ \\
\hline Packet-Loss & 0.6591 & 0.6915 & 0.7115 & $\mathbf{0 . 7 1 8 7}$ \\
\hline White Noise & 0.9048 & 0.9060 & $\mathbf{0 . 9 0 7 9}$ & 0.8947 \\
\hline
\end{tabular}

we combine all videos (from all databases) with the same distortion type into the same group. The accuracy performance of each method is, then, evaluated by comparing the predicted scores with the subjective scores provided by the databases. But, the subjective scores provided by each database were obtained using different experimental methodologies, with different subject pools, and under different physical environments. For example, as described in Chapter 3, the LIVE and IVP databases provide a DMOS for each distorted video, while the CSIQ and MCL-V databases provide a MOS for each distorted video. So, to properly combine these subjective scores into a single scale, we use the Iterated Nested Least-Squares Algorithm (INLSA) [60].

Tables 5.10-5.15 show the results of each method's accuracy performance separated by video 
distortion. The video resolutions vary from $768 \times 432$ to $384 \times 216$. Notice that, for ViS3, STRRED, and SSTS-GMSD, reducing the resolution of videos distorted by MJPEG, packet-loss, and white noise decreases the accuracy performance. Table 5.13 shows the GMSD's accuracy performance for each video distortion. The accuracy performance decreases for videos distorted by white noise, H.265, and MJPEG compression. But the decrease in performance for H.265 Compression $(1.37 \%)$ is lower than for MJPEG and white noise (16.54\% and $8.21 \%)$. Even with this decrease for the videos compressed with H.265, the accuracy performance is still good.

For SSIM and PSNR, when the resolution is reduced, the accuracy performance improves for almost all video distortions. The only exceptions are the videos distorted by white noise and MJPEG. Nevertheless, the accuracy performance of these methods are the worst among all methods, probably because these methods are image quality metrics. If the spatial resolutions of the videos distorted by white noise and MJPEG compression are reduced, the accuracy performances of all video quality methods decrease. For videos distorted by packet-loss, the accuracy performances of ViS3, STRRED, and SSTS-GMSD also decrease.

For these reasons, we use a classification method to identify videos distorted by packet-loss, MPJEG compression, and white noise, and set their resolution to $768 \times 432$. Videos identified as distorted by the other types of distortions should have their spatial resolution reduced to $384 \times 216$. Next, we present the results obtained with the two proposed classifiers.

\subsubsection{Spatial Difference Classifier Results}

The first classifier, based on spatial activity, divided the videos into two groups: videos distorted by packet-loss, MPJEG compression and white noise; and videos distorted by upscaling, H.264, MPEG-2, H.265 and wavelet compression. Table 5.16 shows the accuracy performance of each method using the spatial activity difference classifier. For comparison purposes, we also show the accuracy performance using: (1) the original resolution, (2) all videos in 384x216 and (3) an ideal classification. For the ideal classification, we label all videos according to its distortion and then reduce the spatial resolution to $384 \times 216$ (MJPEG, packet-loss, white noise) or $768 \times 432$ (others distortions). Notice that, for all databases, the accuracy performance of the proposed method is better than using the original resolution, except for STRRED in LIVE and CSIQ.

Analyzing the accuracy performance of the framework using this classifier, we notice that this approach is better than using the videos in the original resolution, for the CSIQ, IVP and MCL-V Databases. For the LIVE Database, the accuracy performance using the classifier is worse than using the videos in the original resolution for ViS3, STRRED, SSTS-GMSD, and GMSD. On the other hand, for SSIM and PSNR the accuracy performance is better using the classifier. The overall accuracy performance of the framework for all methods, when we consider the videos in all databases, is better than using the videos in their original resolution. In some cases, the accuracy performance using the classifier is better than using the ideal classification. This is probably due to influences of the video content. The spatial activity difference classifier is better to identify video contents that are more sensitive to resolution reduction. Finally, the accuracy 
Table 5.16: Spearman Correlation Coefficient (SCC) of all objective methods, using the proposed framework with the Spatial Activity Difference Classifier.

\begin{tabular}{|l|l|l|l|l|l|l|}
\hline Metric & Type of Classification & LIVE & CSIQ & IVP & MCL-V & All \\
\hline ViS3 & Original Resolution & $\mathbf{0 . 8 1 6 8}$ & 0.8325 & 0.7948 & 0.6361 & 0.7466 \\
& All Videos in 384x216 & 0.7595 & 0.7897 & $\mathbf{0 . 8 9 1 2}$ & 0.7674 & 0.8030 \\
& Ideal Classification & 0.7964 & $\mathbf{0 . 8 6 4 6}$ & 0.8905 & 0.7674 & $\mathbf{0 . 8 3 8 1}$ \\
& SA Difference Classification & 0.7610 & 0.8632 & 0.8809 & $\mathbf{0 . 7 7 2 7}$ & 0.8294 \\
\hline STRRED & Original Resolution & $\mathbf{0 . 8 0 0 7}$ & 0.8129 & $\mathbf{0 . 7 3 7 4}$ & 0.7433 & 0.6961 \\
& All Videos in 384x216 & 0.7570 & 0.7424 & 0.7144 & $\mathbf{0 . 8 1 7 7}$ & 0.7774 \\
& Ideal Classification & 0.7954 & $\mathbf{0 . 8 4 6 0}$ & 0.7356 & $\mathbf{0 . 8 1 1 9}$ & $\mathbf{0 . 8 1 7 6}$ \\
& SA Difference Classification & 0.7363 & 0.7622 & 0.6774 & 0.7938 & 0.7698 \\
\hline SSTS-GMSD & Original Resolution & $\mathbf{0 . 8 3 8 9}$ & 0.8415 & 0.7560 & 0.6855 & 0.7403 \\
& All Videos in 384x216 & 0.7777 & 0.7458 & 0.8722 & 0.8000 & 0.7855 \\
& Ideal Classification & 0.7364 & 0.8281 & 0.8699 & 0.8000 & 0.8028 \\
& SA Difference Classification & 0.7720 & $\mathbf{0 . 8 4 9 1}$ & $\mathbf{0 . 8 7 8 0}$ & $\mathbf{0 . 8 0 1 1}$ & $\mathbf{0 . 8 2 5 0}$ \\
\hline \multirow{3}{*}{ GMSD } & Original Resolution & 0.7262 & 0.8409 & 0.6924 & 0.6449 & 0.6781 \\
& All Videos in 384x216 & $\mathbf{0 . 7 2 6 4}$ & 0.7729 & 0.8672 & 0.7708 & 0.7874 \\
& Ideal Classification & 0.6787 & 0.8475 & 0.8611 & 0.7708 & 0.8020 \\
& SA Difference Classification & 0.7217 & $\mathbf{0 . 8 6 0 3}$ & $\mathbf{0 . 8 7 0 8}$ & $\mathbf{0 . 7 7 1 8}$ & $\mathbf{0 . 8 2 2 5}$ \\
\hline SSIM & Original Resolution & 0.5251 & 0.5794 & 0.3560 & 0.4018 & 0.4833 \\
& All Videos in 384x216 & $\mathbf{0 . 7 0 0 2}$ & 0.7241 & 0.6965 & $\mathbf{0 . 7 1 0 5}$ & $\mathbf{0 . 7 3 3 3}$ \\
& Ideal Classification & 0.6567 & 0.6728 & $\mathbf{0 . 7 6 2 4}$ & $\mathbf{0 . 7 1 0 5}$ & 0.7217 \\
& SA Difference Classification & 0.6849 & $\mathbf{0 . 7 3 7 3}$ & 0.6775 & 0.6613 & 0.7324 \\
\hline PSNR & Original Resolution & 0.5233 & 0.5787 & 0.6566 & 0.4640 & 0.5795 \\
& All Videos in 384x216 & $\mathbf{0 . 6 2 8 5}$ & 0.5628 & 0.8374 & $\mathbf{0 . 6 8 7 6}$ & 0.6857 \\
& Ideal Classification & 0.6239 & $\mathbf{0 . 6 2 8 4}$ & $\mathbf{0 . 8 6 6 3}$ & $\mathbf{0 . 6 8 7 6}$ & $\mathbf{0 . 7 1 3 2}$ \\
& SA Difference Classification & 0.6158 & 0.5974 & 0.8039 & 0.5964 & 0.6879 \\
\hline
\end{tabular}

performance for videos in $384 \times 216$ resolution, i.e. if we reduce the resolution regardless the distortion, is worse than using the framework classifier, except for SSIM. Therefore, if we want to improve the running time of a video quality assessment method, while maintaining the accuracy performance, we have to identify the video distortion and reduce its resolution accordingly.

From the SCC of the video quality assessment methods, we know that the predicted scores obtained via the proposed framework are more correlated with subjective scores than the predicted scores obtained without using the framework. However, it is important to evaluate if the differences are statistically significant. To evaluate the statistical significance of the differences in accuracy performance with and without the framework, we perform a t-test on the SCC values obtained in 1,000 simulations [61]. In these simulations, we randomly choose $80 \%$ of the reference videos and their distorted versions for each simulation. The results of the t-test are shown in Table 5.17. The value " 1 " in the table indicates that the method in the row is statistically superior to the method in the column, while the value "-1" indicates that the method in the row is statistically inferior to the method in the column. Finally, the value " 0 " indicates that both methods are statistically indistinguishable. Notice that for the overall accuracy performance, considering all databases, the improvement using the framework with the spatial activity classifier is statistically significant. Figure 5.1 shows the box plot of the SCC across the 1000 simulations. 


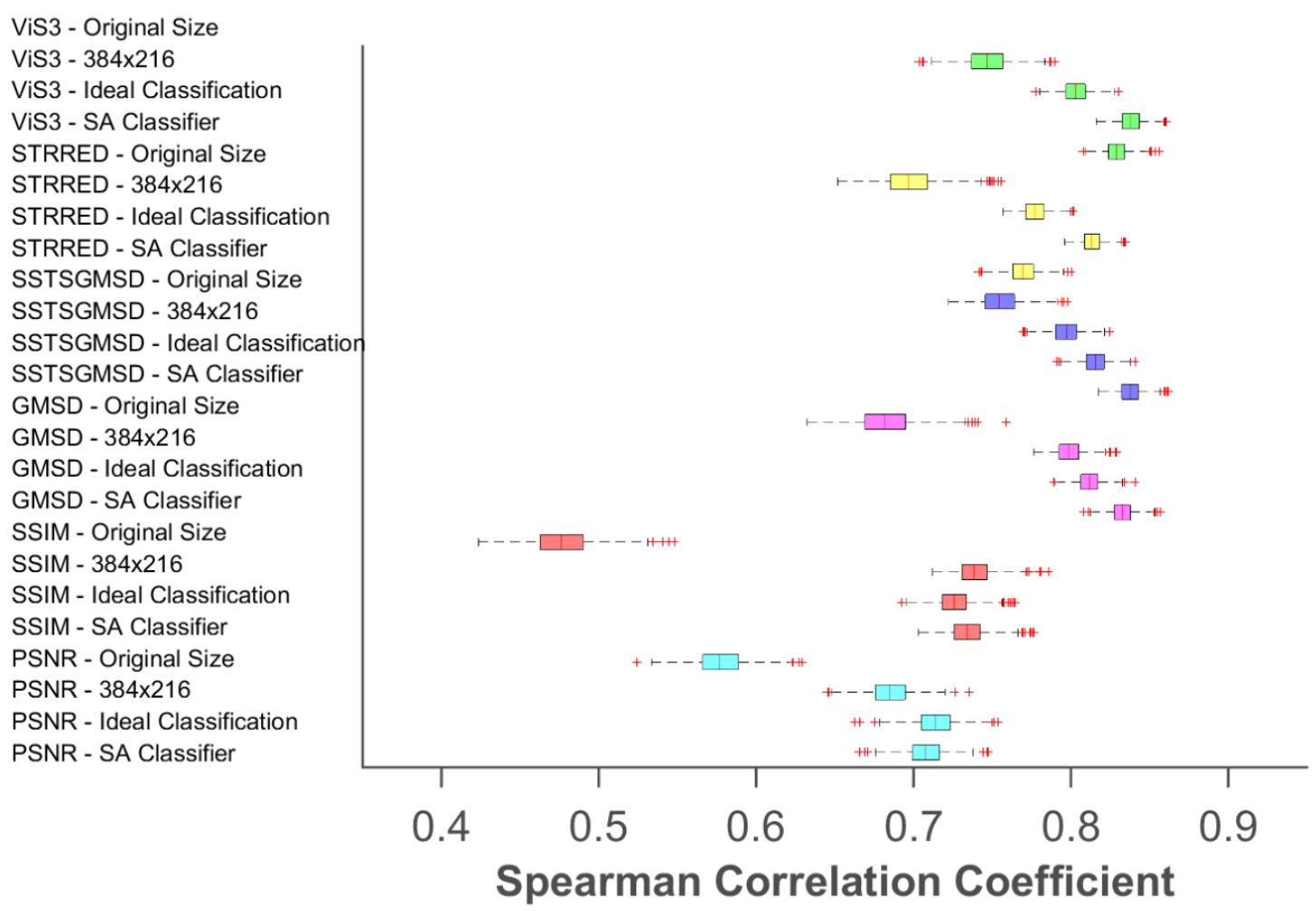

Figure 5.1: Box plot of SCC across 1000 simulations, where we ramdonly chose $80 \%$ of the reference videos and their distortions versions.

As said in the beginning of this chapter, the objective of reducing the video resolution is to reduce the running time of the video quality assessment method. The proposed classifier has the goal of identifying which videos can have its resolution reduced, avoiding a decrease in accuracy performance of the video quality assessment method. As mentioned in Chapter 4, we proposed a methodology to evaluate the video quality, composed of three stages. The first stage consist of identifying video distortion. The second stage reduces the video resolution to the smallest possible resolution, according to the video distortion. The third stage adjusts the predicted quality score, according to the video resolution. From the results in Table 5.16, we know that using the framework is a good way to maintain the accuracy performance of a quality assessment method. But, if we want to improve the runtime performance of video quality assessment methods, the running time of the classifier plus the running time of these methods has to be smaller than the running time of the video quality assessment method using the video in its original spatial resolution. Table 5.18 presents the average running time of the video quality assessment methods ran on videos in their original resolution and on videos with the resolution reduced, using the proposed methodology with the spatial difference classifier. From these results, we notice that, even adding the time spent to identify the video resolution and resizing the video, the proposed methodology is faster than the original video quality assessment method. When image quality assessment methods (GMSD,SSIM and PSNR) are used to measure video quality, the improvement in running time is small (SSIM is $1.17 \mathrm{x}$ faster, GMSD is $1.11 \mathrm{x}$ faster). Nevertheless, for more complex video quality assessment methods, the improvement in time is considerable (ViS3 is $2.85 \mathrm{x}$ faster, STRRED is $2.29 \mathrm{x}$ faster and SSTS-GMSD is $1.51 \mathrm{x}$ faster). 
Table 5.17: T-Test results between the SCC of various video quality assessment methods for all databases. The video quality assessment methods evaluate the videos in their original spatial resolution (Ori), 384x216 resolution (Min), using the framework with an ideal classifier (Ideal), and with SA classifier (SA).

\begin{tabular}{|c|c|c|c|c|c|c|c|c|c|c|c|c|c|}
\hline & \multicolumn{4}{|c|}{ ViS3 } & \multicolumn{4}{|c|}{ STRRED } & \multicolumn{4}{|c|}{ SSTS-GMSD } \\
\hline & & Ori & Min & Ideal & SA & Ori & Min & Ideal & SA & Ori & Min & Ideal & SA \\
\hline \multirow[t]{4}{*}{ ViS3 } & Ori & 0 & -1 & -1 & -1 & 1 & -1 & -1 & -1 & 0 & -1 & -1 & -1 \\
\hline & Min & 1 & 0 & -1 & -1 & 1 & 0 & -1 & -1 & 1 & -1 & -1 & -1 \\
\hline & Ideal & 1 & 1 & 0 & 1 & 1 & 1 & 1 & 1 & 1 & 1 & -1 & -1 \\
\hline & SA & 1 & 1 & -1 & 0 & 1 & 1 & -1 & 1 & 1 & -1 & -1 & -1 \\
\hline \multirow[t]{4}{*}{ STRRED } & Ori & -1 & -1 & -1 & -1 & 0 & -1 & -1 & -1 & -1 & -1 & -1 & -1 \\
\hline & Min & 1 & 0 & -1 & -1 & 1 & 0 & -1 & -1 & 1 & -1 & -1 & -1 \\
\hline & Ideal & 1 & 1 & -1 & 1 & 1 & 1 & 0 & 0 & 1 & 1 & -1 & -1 \\
\hline & SA & 1 & 1 & -1 & -1 & 1 & 1 & 0 & 0 & 1 & -1 & -1 & -1 \\
\hline \multirow[t]{4}{*}{ SSTSGMSD } & Ori & 0 & -1 & -1 & -1 & 1 & -1 & -1 & -1 & 0 & -1 & -1 & -1 \\
\hline & Min & 1 & 1 & -1 & 1 & 1 & 1 & -1 & 1 & 1 & 0 & -1 & -1 \\
\hline & Ideal & 1 & 1 & 1 & 1 & 1 & 1 & 1 & 1 & 1 & 1 & 0 & 1 \\
\hline & SA & 1 & 1 & 1 & 1 & 1 & 1 & 1 & 1 & 1 & 1 & -1 & 0 \\
\hline \multirow[t]{4}{*}{ GSMD } & Ori & -1 & -1 & -1 & -1 & -1 & -1 & -1 & -1 & -1 & -1 & -1 & -1 \\
\hline & Min & 1 & 1 & -1 & -1 & 1 & 1 & -1 & -1 & 1 & -1 & -1 & -1 \\
\hline & Ideal & 1 & 1 & 1 & 1 & 1 & 1 & 1 & 1 & 1 & 1 & -1 & -1 \\
\hline & SA & 1 & 1 & 1 & 1 & 1 & 1 & 1 & 1 & 1 & 1 & -1 & -1 \\
\hline \multirow[t]{4}{*}{ SSIM } & Ori & -1 & -1 & -1 & -1 & -1 & -1 & -1 & -1 & -1 & -1 & -1 & -1 \\
\hline & Min & 1 & -1 & -1 & -1 & 1 & -1 & -1 & -1 & 1 & -1 & -1 & -1 \\
\hline & Ideal & 1 & -1 & -1 & -1 & 1 & -1 & -1 & -1 & 1 & -1 & -1 & -1 \\
\hline & SA & 0 & -1 & -1 & -1 & 1 & -1 & -1 & -1 & 1 & -1 & -1 & -1 \\
\hline \multirow[t]{4}{*}{ PSNR } & Ori & -1 & -1 & -1 & -1 & -1 & -1 & -1 & -1 & -1 & -1 & -1 & -1 \\
\hline & Min & -1 & -1 & -1 & -1 & 1 & -1 & -1 & -1 & -1 & -1 & -1 & -1 \\
\hline & Ideal & -1 & -1 & -1 & -1 & 1 & -1 & -1 & -1 & -1 & -1 & -1 & -1 \\
\hline & SA & -1 & -1 & -1 & -1 & 0 & -1 & -1 & -1 & -1 & -1 & -1 & -1 \\
\hline & & \multicolumn{4}{|c|}{ GMSD } & \multicolumn{4}{|c|}{ "SSIM } & \multicolumn{4}{|c|}{ PSNR } \\
\hline & & Ori & Min & Ideal & SA & Ori & Min & Ideal & SA & Ori & Min & Ideal & SA \\
\hline \multirow[t]{4}{*}{ ViS3 } & Ori & 1 & -1 & -1 & -1 & 1 & -1 & -1 & 0 & 1 & 1 & 1 & 1 \\
\hline & Min & 1 & -1 & -1 & -1 & 1 & 1 & 1 & 1 & 1 & 1 & 1 & 1 \\
\hline & Ideal & 1 & 1 & -1 & -1 & 1 & 1 & 1 & 1 & 1 & 1 & 1 & 1 \\
\hline & SA & 1 & 1 & -1 & -1 & 1 & 1 & 1 & 1 & 1 & 1 & 1 & 1 \\
\hline \multirow[t]{4}{*}{ STRRED } & Ori & 1 & -1 & -1 & -1 & 1 & -1 & -1 & -1 & 1 & -1 & -1 & 0 \\
\hline & Min & 1 & -1 & -1 & -1 & 1 & 1 & 1 & 1 & 1 & 1 & 1 & 1 \\
\hline & Ideal & 1 & 1 & -1 & -1 & 1 & 1 & 1 & 1 & 1 & 1 & 1 & 1 \\
\hline & SA & 1 & 1 & -1 & -1 & 1 & 1 & 1 & 1 & 1 & 1 & 1 & 1 \\
\hline \multirow[t]{4}{*}{ SSTSGMSD } & Ori & 1 & -1 & -1 & -1 & 1 & -1 & -1 & -1 & 1 & 1 & 1 & 1 \\
\hline & Min & 1 & 1 & -1 & -1 & 1 & 1 & 1 & 1 & 1 & 1 & 1 & 1 \\
\hline & Ideal & 1 & 1 & 1 & 1 & 1 & 1 & 1 & 1 & 1 & 1 & 1 & 1 \\
\hline & SA & 1 & 1 & 1 & 1 & 1 & 1 & 1 & 1 & 1 & 1 & 1 & 1 \\
\hline \multirow[t]{4}{*}{ GSMD } & Ori & 0 & -1 & -1 & -1 & 1 & -1 & -1 & -1 & 1 & -1 & -1 & -1 \\
\hline & Min & 1 & 0 & -1 & -1 & 1 & 1 & 1 & 1 & 1 & 1 & 1 & 1 \\
\hline & Ideal & 1 & 1 & 0 & 1 & 1 & 1 & 1 & 1 & 1 & 1 & 1 & 1 \\
\hline & SA & 1 & 1 & -1 & 0 & 1 & 1 & 1 & 1 & 1 & 1 & 1 & 1 \\
\hline SSIM & Ori & -1 & -1 & -1 & -1 & 0 & -1 & -1 & -1 & -1 & -1 & -1 & -1 \\
\hline & Min & 1 & -1 & -1 & -1 & 1 & 0 & -1 & 1 & 1 & 1 & 1 & 1 \\
\hline & Ideal & 1 & -1 & -1 & -1 & 1 & 1 & 0 & 1 & 1 & 1 & 1 & 1 \\
\hline & SA & 1 & -1 & -1 & -1 & 1 & -1 & -1 & 0 & 1 & 1 & 1 & 1 \\
\hline PSNR & Ori & -1 & -1 & -1 & -1 & 1 & -1 & -1 & -1 & 0 & -1 & -1 & -1 \\
\hline & Min & 1 & -1 & -1 & -1 & 1 & -1 & -1 & -1 & 1 & 0 & -1 & 1 \\
\hline & Ideal & 1 & -1 & -1 & -1 & 1 & -1 & -1 & -1 & 1 & 1 & 0 & 1 \\
\hline & SA & 1 & -1 & -1 & -1 & 1 & -1 & -1 & -1 & 1 & -1 & -1 & 0 \\
\hline
\end{tabular}


Table 5.18: Average running time for performing an objective quality assessment with and without spatial activity classifier (in seconds).

\begin{tabular}{|l|l|l|l|l|l|l|}
\cline { 2 - 7 } \multicolumn{1}{c|}{} & ViS3 & STRRED & SSTS-GMSD & SSIM & GMSD & PNSR \\
\hline Original Resolution & 639.135 & 215.973 & 13.557 & 13.108 & 5.951 & 5.033 \\
\hline SA Difference Classifier & 223.784 & 94.384 & 8.911 & 11.221 & 5.330 & 5.029 \\
\hline
\end{tabular}

\subsubsection{SSEQ Classifier Results}

The SSEQ Classifier uses a machine learning technique to classify the videos according to their distortion. The videos in all databases are divided in training and testing groups. $80 \%$ of the reference videos and their associated distorted versions were used for training and $20 \%$ of the reference videos and their associated distorted versions were used for testing. We use a CSVM with linear kernel, as it was done in the original SSEQ algorithm for images. We combine videos in all databases and divided the whole set in training and testing groups. The training group consists of 472 to 480 video sequences, from which 120 video sequences are from LIVE Database, 180 video sequences are from CSIQ Database, 72 video sequences are from MCL-V Database and 100 to 108 video sequences are from IVP. Some reference video sequences in IVP

Table 5.19: Median Spearman Correlation Coefficient of all methods using SSEQ Distortion Classifier in 1000 simulations.

\begin{tabular}{|l|l|l|l|l|l|l|}
\hline Metric & Type of Classification & LIVE & CSIQ & IVP & MCL-V & All \\
\hline ViS3 & Original Resolution & $\mathbf{0 . 8 4 9 9}$ & 0.8574 & 0.8470 & 0.5882 & 0.7532 \\
& All Videos with 384x216 & 0.7740 & 0.8085 & $\mathbf{0 . 9 0 1 4}$ & $\mathbf{0 . 6 7 6 5}$ & 0.7953 \\
& Ideal Classification & 0.8202 & $\mathbf{0 . 8 7 3 6}$ & 0.8998 & $\mathbf{0 . 6 7 6 5}$ & $\mathbf{0 . 8 2 9 4}$ \\
& Predicted Classification & 0.7882 & 0.8521 & 0.8946 & $\mathbf{0 . 6 7 6 5}$ & 0.8164 \\
\hline STRRED & Original Resolution & $\mathbf{0 . 8 2 6 3}$ & 0.8093 & 0.7583 & $\mathbf{0 . 9 9 4 1}$ & 0.7061 \\
& All Videos with 384x216 & 0.7873 & 0.7565 & $\mathbf{0 . 7 9 3 1}$ & 0.9853 & 0.7957 \\
& Ideal Classification & 0.7941 & $\mathbf{0 . 8 3 3 1}$ & 0.7748 & 0.9853 & $\mathbf{0 . 8 2 6 3}$ \\
& Predicted Classification & 0.7931 & 0.8055 & 0.7871 & 0.9853 & 0.8136 \\
\hline SSTS-GMSD & Original Resolution & $\mathbf{0 . 8 6 2 5}$ & 0.8389 & 0.7600 & 0.7529 & 0.7526 \\
& All Videos with 384x216 & 0.8327 & 0.7961 & $\mathbf{0 . 9 1 4 6}$ & $\mathbf{0 . 9 1 1 8}$ & 0.8230 \\
& Ideal Classification & 0.8411 & $\mathbf{0 . 8 7 4 1}$ & 0.8971 & $\mathbf{0 . 9 1 1 8}$ & $\mathbf{0 . 8 5 0 1}$ \\
& Predicted Classification & 0.8314 & 0.8565 & 0.9078 & $\mathbf{0 . 9 1 1 8}$ & 0.8430 \\
\hline GMSD & Original Resolution & $\mathbf{0 . 7 9 6 7}$ & 0.8636 & 0.6705 & 0.7529 & 0.6757 \\
& All Videos with384x216 & 0.7784 & 0.7761 & $\mathbf{0 . 8 7 5 7}$ & $\mathbf{0 . 9 1 1 8}$ & 0.8045 \\
& Ideal Classification & 0.7850 & $\mathbf{0 . 8 8 6 2}$ & 0.8703 & $\mathbf{0 . 9 1 1 8}$ & $\mathbf{0 . 8 4 1 9}$ \\
& Predicted Classification & 0.7780 & 0.8644 & 0.8706 & $\mathbf{0 . 9 1 1 8}$ & 0.8347 \\
\hline SSIM & Original Resolution & 0.6535 & 0.6039 & 0.4105 & 0.4618 & 0.4877 \\
& All Videos with384x216 & $\mathbf{0 . 8 1 6 7}$ & 0.7426 & 0.7597 & $\mathbf{0 . 9 6 1 8}$ & 0.7715 \\
& Ideal Classification & 0.7375 & $\mathbf{0 . 7 7 6 4}$ & $\mathbf{0 . 8 3 3 1}$ & $\mathbf{0 . 9 6 1 8}$ & $\mathbf{0 . 7 9 0 6}$ \\
& Predicted Classification & 0.7595 & 0.7282 & 0.7477 & $\mathbf{0 . 9 6 1 8}$ & 0.7585 \\
\hline PSNR & Original Resolution & 0.7112 & 0.6162 & 0.7187 & 0.5118 & 0.6052 \\
& All Videos with384x216 & $\mathbf{0 . 7 5 1 7}$ & 0.5887 & 0.8238 & $\mathbf{0 . 9 2 9 4}$ & 0.7204 \\
& Ideal Classification & 0.6934 & $\mathbf{0 . 6 4 8 9}$ & $\mathbf{0 . 8 6 5 6}$ & $\mathbf{0 . 9 2 9 4}$ & $\mathbf{0 . 7 4 0 2}$ \\
& Predicted Classification & 0.6211 & 0.6108 & 0.8446 & $\mathbf{0 . 9 2 9 4}$ & 0.7028 \\
\hline
\end{tabular}


Database do not have Packet-Loss video sequences associated, that is why the number of video sequences in the train sets varies. The test set consist of 110 to 118 video sequences, from which 30 video sequences are from LIVE Database, 36 video sequences are from CSIQ Database, 24 video sequences are from MCL-V Database and 20 to 28 video sequences are from IVP. The training and testing sets do not share test sequences corresponding to the same content (original). In summary, for 1,000 simulations, the databases are randomly divided in traning and testing groups, with no overlap of content. Table 5.19 shows the median of the SCC values obtained for these 1,000 simulations.

We compare the results obtained with the SSEQ classifier with the results obtained with the videos in the original resolution, the videos in $384 \times 216$ resolution, and the videos with spatial resolution chosen using an ideal classification. Figure 5.2 shows the box plot of SCC across 1,000 simulations for the overall accuracy performance, considering the videos of all databases. Notice that using the ideal classification generates the best results.

The gain in accuracy performance using the ideal classification is $10.14 \%$ for ViS3, $17.30 \%$ for STRRED, $13.32 \%$ for SSTS-GMSD, $25.01 \%$ for GMSD, $60.85 \%$ for SSIM, and $22.17 \%$ for PSNR. The SSEQ classifier gives the overall second best, with the exception of SSIM and PSNR. The gain in accuracy performance using the SSEQ classifier is $8.37 \%$ for ViS3, $15.70 \%$ for STRRED, 12.64\% for SSTS-GMSD, 23.94\% for GMSD, 53.74\% for SSIM, and $15.96 \%$ for PSNR. The highest gains correspond to the MCL-V and IVP databases, which have the videos with a higher resolution. Also, the gain in accuracy performance of image quality methods is

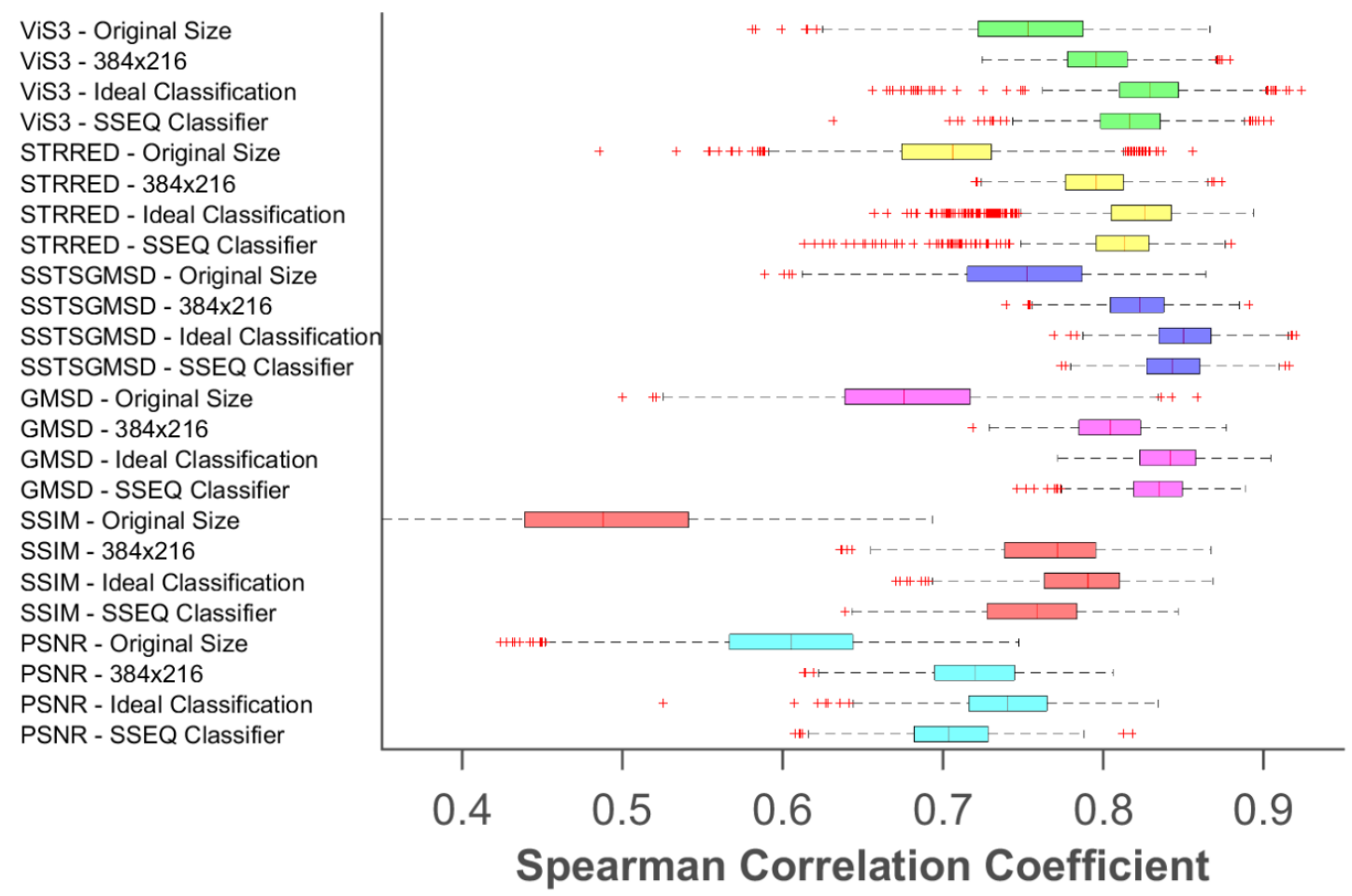

Figure 5.2: Box plot of SCC across 1000 test simulations, where we ramdonly chose $20 \%$ of the reference videos and their distortions versions. 
Table 5.20: T-Test results between the SCC of various video quality assessment methods for all databases. The video quality assessment methods evaluate the videos in their original spatial resolution (Ori), 384x216 resolution (Min), using the framework with an ideal classifier (Ideal), and with SSEQ classifier (SSEQ).

\begin{tabular}{|c|c|c|c|c|c|c|c|c|c|c|c|c|c|}
\hline & \multicolumn{4}{|c|}{ ViS3 } & \multicolumn{4}{|c|}{ STRRED } & \multicolumn{4}{|c|}{ STS-GMSD } \\
\hline & & Ori & Min & Ideal & SSEQ & Ori & Min & Ideal & SSEQ & Ori & Min & Ideal & SSEQ \\
\hline \multirow[t]{4}{*}{ ViS3 } & Ori & 0 & -1 & -1 & -1 & 1 & -1 & -1 & -1 & 0 & -1 & -1 & -1 \\
\hline & Min & 1 & 0 & -1 & -1 & 1 & 0 & -1 & -1 & 1 & -1 & -1 & -1 \\
\hline & Ideal & 1 & 1 & 0 & 1 & 1 & 1 & 1 & 1 & 1 & 1 & -1 & -1 \\
\hline & SSEQ & 1 & 1 & -1 & 0 & 1 & 1 & -1 & 1 & 1 & -1 & -1 & -1 \\
\hline \multirow[t]{4}{*}{ STRRED } & Ori & -1 & -1 & -1 & -1 & 0 & -1 & -1 & -1 & -1 & -1 & -1 & -1 \\
\hline & Min & 1 & 0 & -1 & -1 & 1 & 0 & -1 & -1 & 1 & -1 & -1 & -1 \\
\hline & Ideal & 1 & 1 & -1 & 1 & 1 & 1 & 0 & 0 & 1 & 1 & -1 & -1 \\
\hline & SSEQ & 1 & 1 & -1 & -1 & 1 & 1 & 0 & 0 & 1 & -1 & -1 & -1 \\
\hline \multirow[t]{4}{*}{ SSTSGMSD } & Ori & 0 & -1 & -1 & -1 & 1 & -1 & -1 & -1 & 0 & -1 & -1 & -1 \\
\hline & Min & 1 & 1 & -1 & 1 & 1 & 1 & -1 & 1 & 1 & 0 & -1 & -1 \\
\hline & Ideal & 1 & 1 & 1 & 1 & 1 & 1 & 1 & 1 & 1 & 1 & 0 & 1 \\
\hline & SSEQ & 1 & 1 & 1 & 1 & 1 & 1 & 1 & 1 & 1 & 1 & -1 & 0 \\
\hline \multirow[t]{4}{*}{ GSMD } & Ori & -1 & -1 & -1 & -1 & -1 & -1 & -1 & -1 & -1 & -1 & -1 & -1 \\
\hline & Min & 1 & 1 & -1 & -1 & 1 & 1 & -1 & -1 & 1 & -1 & -1 & -1 \\
\hline & Ideal & 1 & 1 & 1 & 1 & 1 & 1 & 1 & 1 & 1 & 1 & -1 & -1 \\
\hline & SSEQ & 1 & 1 & 1 & 1 & 1 & 1 & 1 & 1 & 1 & 1 & -1 & -1 \\
\hline \multirow{4}{*}{ SSIM } & Ori & -1 & -1 & -1 & -1 & -1 & -1 & -1 & -1 & -1 & -1 & -1 & -1 \\
\hline & Min & 1 & -1 & -1 & -1 & 1 & -1 & -1 & -1 & 1 & -1 & -1 & -1 \\
\hline & Ideal & 1 & -1 & -1 & -1 & 1 & -1 & -1 & -1 & 1 & -1 & -1 & -1 \\
\hline & SSEQ & 0 & -1 & -1 & -1 & 1 & -1 & -1 & -1 & 1 & -1 & -1 & -1 \\
\hline \multirow[t]{4}{*}{ PSNR } & Ori & -1 & -1 & -1 & -1 & -1 & -1 & -1 & -1 & -1 & -1 & -1 & -1 \\
\hline & Min & -1 & -1 & -1 & -1 & 1 & -1 & -1 & -1 & -1 & -1 & -1 & -1 \\
\hline & Ideal & -1 & -1 & -1 & -1 & 1 & -1 & -1 & -1 & -1 & -1 & -1 & -1 \\
\hline & SSEQ & -1 & -1 & -1 & -1 & 0 & -1 & -1 & -1 & -1 & -1 & -1 & -1 \\
\hline & & \multicolumn{4}{|c|}{ GMSD } & \multicolumn{4}{|c|}{ SSIM } & \multicolumn{4}{|c|}{ PSNR } \\
\hline & & Ori & Min & Ideal & SSEQ & Ori & Min & Ideal & SSEQ & Ori & Min & Ideal & SSEQ \\
\hline \multirow[t]{4}{*}{ ViS3 } & Ori & 1 & -1 & -1 & -1 & 1 & -1 & -1 & 0 & 1 & 1 & 1 & 1 \\
\hline & Min & 1 & -1 & -1 & -1 & 1 & 1 & 1 & 1 & 1 & 1 & 1 & 1 \\
\hline & Ideal & 1 & 1 & -1 & -1 & 1 & 1 & 1 & 1 & 1 & 1 & 1 & 1 \\
\hline & SSEQ & 1 & 1 & -1 & -1 & 1 & 1 & 1 & 1 & 1 & 1 & 1 & 1 \\
\hline \multirow[t]{4}{*}{ STRRED } & Ori & 1 & -1 & -1 & -1 & 1 & -1 & -1 & -1 & 1 & -1 & -1 & 0 \\
\hline & Min & 1 & -1 & -1 & -1 & 1 & 1 & 1 & 1 & 1 & 1 & 1 & 1 \\
\hline & Ideal & 1 & 1 & -1 & -1 & 1 & 1 & 1 & 1 & 1 & 1 & 1 & 1 \\
\hline & SSEQ & 1 & 1 & -1 & -1 & 1 & 1 & 1 & 1 & 1 & 1 & 1 & 1 \\
\hline \multirow[t]{4}{*}{ SSTSGMSD } & Ori & 1 & -1 & -1 & -1 & 1 & -1 & -1 & -1 & 1 & 1 & 1 & 1 \\
\hline & Min & 1 & 1 & -1 & -1 & 1 & 1 & 1 & 1 & 1 & 1 & 1 & 1 \\
\hline & Ideal & 1 & 1 & 1 & 1 & 1 & 1 & 1 & 1 & 1 & 1 & 1 & 1 \\
\hline & SSEQ & 1 & 1 & 1 & 1 & 1 & 1 & 1 & 1 & 1 & 1 & 1 & 1 \\
\hline \multirow[t]{4}{*}{ GSMD } & Ori & 0 & -1 & -1 & -1 & 1 & -1 & -1 & -1 & 1 & -1 & -1 & -1 \\
\hline & Min & 1 & 0 & -1 & -1 & 1 & 1 & 1 & 1 & 1 & 1 & 1 & 1 \\
\hline & Ideal & 1 & 1 & 0 & 1 & 1 & 1 & 1 & 1 & 1 & 1 & 1 & 1 \\
\hline & SSEQ & 1 & 1 & -1 & 0 & 1 & 1 & 1 & 1 & 1 & 1 & 1 & 1 \\
\hline SSIM & Ori & -1 & -1 & -1 & -1 & 0 & -1 & -1 & -1 & -1 & -1 & -1 & -1 \\
\hline & Min & 1 & -1 & -1 & -1 & 1 & 0 & -1 & 1 & 1 & 1 & 1 & 1 \\
\hline & Ideal & 1 & -1 & -1 & -1 & 1 & 1 & 0 & 1 & 1 & 1 & 1 & 1 \\
\hline & SSEQ & 1 & -1 & -1 & -1 & 1 & -1 & -1 & 0 & 1 & 1 & 1 & 1 \\
\hline PSNR & Ori & -1 & -1 & -1 & -1 & 1 & -1 & -1 & -1 & 0 & -1 & -1 & -1 \\
\hline & Min & 1 & -1 & -1 & -1 & 1 & -1 & -1 & -1 & 1 & 0 & -1 & 1 \\
\hline & Ideal & 1 & -1 & -1 & -1 & 1 & -1 & -1 & -1 & 1 & 1 & 0 & 1 \\
\hline & SSEQ & 1 & -1 & -1 & -1 & 1 & -1 & -1 & -1 & 1 & -1 & -1 & 0 \\
\hline
\end{tabular}


Table 5.21: Average running time for performing an objective quality assessment with and without SSEQ classifier (in seconds).

\begin{tabular}{|l|l|l|l|l|l|l|}
\cline { 2 - 7 } \multicolumn{1}{c|}{} & ViS3 & STRRED & SSTS-GMSD & SSIM & GMSD & PNSR \\
\hline Original Resolution & 647.5480 & 219.9712 & 13.7975 & 13.6208 & 6.1423 & 5.1625 \\
\hline SA Difference Classification & 278.4741 & 157.7950 & 73.9999 & 76.2293 & 70.5274 & 70.2187 \\
\hline
\end{tabular}

higher than of video quality methods. And, when using the SSEQ classifier, SSTS-GMSD has the second best accuracy performance.

The statistical significance of the differences in accuracy performance was made using a ttest with 1,000 simulations. Tables 5.20 shows the results of the t-test. The improvement when using the framework with the SSEQ classifier is statistically significant. Notice that the results of STRRED using the ideal classifier and of the SSEQ classifier are equivalent, even though the SCC of STRRED with ideal classifier is greater than the SCC of STRRED with SSEQ classifier. For the videos with original resolution, ViS3 has the best accurary performance among all video quality assessment methods tested. It is worth noticing that all methods using the framework, except for PSNR, have a better accuracy performance than the ViS3 on videos with original resolution, which is the best method accuracy performance without using the framework.

Table 5.21 presents the average running time for video quality assessment methods for videos in their original resolution and with the proposed methodology using the SSEQ classifier. Unfortunately, the SSEQ classifier is slower than the SA Classifier, because it extracts more features from the video. Only the running time of ViS3 and STRRED is improved when using the SSEQ classifier (ViS3 is $2.32 \mathrm{x}$ faster and STRRED is $1.39 \mathrm{x}$ faster).

\subsubsection{Influence of Frame Rate in Video Quality Metrics}

Other way to improve the runtime performance of video quality assessment methods is to reduce the frame rate of the videos. Table 5.22 shows the SCC of the video quality assessment methods, when reducing the video frame rate to $24,16,8$, and 4 FPS. There are no ViS3 results for videos with 4 FPS in MCL-V database, because these videos do not have the temporal information required by ViS3. From these results, we notice that the ViS3 and STRRED accuracy performances are better with a higher FPS. We believe this happens because these algorithms analyze the temporal information of the video to measure its quality. For example, ViS3 uses motion vectors to give weight to specific areas in the spatial distortion map. Therefore, in this case, by reducing the temporal resolution we might introduce temporal transitions to the video. STRRED, on the other hand, analyses the differences among neighboring frames in the video. Therefore, if there is a large time interval between to two consecutive frames, there will be a large temporal difference between them. Nevertheless, for these two video quality assessment methods, we can reduce the FPS to 16 FPS, without greatly affecting the accuracy performance.

For LIVE and CSIQ databases, the accuracy performance of SSTS-GMSD increases with higher FPS. And, for IVP and MCL-V databases, the accuracy performance of SSTS-GMSD de- 
Table 5.22: Spearman Coefficient Correlation of the methods when varying the video FPS.

\begin{tabular}{|l|l|l|l|l|l|l|}
\hline \multirow{2}{*}{ Métrica } & Banco & \multicolumn{5}{|c|}{ FPS } \\
\cline { 2 - 7 } & de Dados & Original & 24 & 16 & 8 & 4 \\
\hline \multirow{5}{*}{ ViS3 } & LIVE & 0.8168 & $\mathbf{0 . 8 1 9 1}$ & 0.8108 & 0.8021 & 0.7586 \\
& CSIQ & 0.8325 & $\mathbf{0 . 8 3 7 9}$ & 0.8347 & 0.8040 & 0.7573 \\
& IVP & 0.7948 & 0.7931 & $\mathbf{0 . 7 9 9 8}$ & 0.7439 & 0.7466 \\
& MCL-V & 0.6361 & 0.6500 & $\mathbf{0 . 6 9 3 3}$ & 0.6693 & - \\
\hline \multirow{5}{*}{ STRRED } & LIVE & 0.8007 & $\mathbf{0 . 8 0 7 4}$ & 0.8002 & 0.7888 & 0.7773 \\
\cline { 2 - 7 } & CSIQ & $\mathbf{0 . 8 1 2 9}$ & 0.7995 & 0.7964 & 0.7799 & 0.7617 \\
\cline { 2 - 7 } & IVP & 0.7374 & 0.7396 & $\mathbf{0 . 7 4 7 5}$ & 0.7403 & 0.7393 \\
\cline { 2 - 7 } & MCL-V & $\mathbf{0 . 7 4 3 3}$ & 0.7431 & 0.7381 & 0.7281 & 0.7222 \\
\hline \multirow{5}{*}{ SSTS-GMSD } & LIVE & $\mathbf{0 . 8 3 8 9}$ & 0.8262 & 0.8182 & 0.7967 & 0.7813 \\
& CSIQ & 0.8415 & $\mathbf{0 . 8 4 9 2}$ & 0.8474 & 0.8430 & 0.8299 \\
& IVP & 0.7560 & 0.7490 & 0.7553 & 0.7662 & $\mathbf{0 . 7 8 6 3}$ \\
& MCL-V & 0.6855 & 0.6881 & 0.6937 & 0.6932 & $\mathbf{0 . 7 0 0 8}$ \\
\hline \multirow{5}{*}{ GMSD } & LIVE & 0.7262 & 0.7311 & 0.7108 & $\mathbf{0 . 7 3 5 8}$ & 0.6900 \\
\cline { 2 - 7 } & CSIQ & $\mathbf{0 . 8 4 0 9}$ & 0.8391 & 0.8390 & 0.8383 & 0.8364 \\
\cline { 2 - 7 } & IVP & $\mathbf{0 . 6 9 2 4}$ & 0.6839 & 0.6785 & 0.6894 & 0.6691 \\
\cline { 2 - 7 } & MCL-V & 0.6449 & 0.6490 & 0.6477 & 0.6514 & $\mathbf{0 . 6 6 0 8}$ \\
\hline \multirow{5}{*}{ SSIM } & LIVE & 0.5251 & 0.5210 & 0.5010 & $\mathbf{0 . 5 4 0 0}$ & 0.5137 \\
& CSIQ & 0.5794 & 0.5793 & 0.5786 & $\mathbf{0 . 5 8 5 2}$ & 0.5765 \\
& IVP & 0.3560 & 0.3556 & 0.3493 & $\mathbf{0 . 3 5 6 8}$ & 0.3404 \\
& MCL-V & 0.4018 & 0.4009 & 0.4050 & 0.3976 & $\mathbf{0 . 4 0 6 6}$ \\
\hline \multirow{5}{*}{ PSNR } & LIVE & 0.5251 & 0.5096 & 0.4921 & 0.5348 & $\mathbf{0 . 5 3 7 6}$ \\
\cline { 2 - 7 } & CSIQ & 0.5794 & 0.5791 & 0.5761 & 0.5842 & $\mathbf{0 . 5 8 4 7}$ \\
\cline { 2 - 7 } & IVP & $\mathbf{0 . 6 5 6 6}$ & 0.6469 & 0.6376 & 0.6501 & 0.6274 \\
\cline { 2 - 7 } & MCL-V & 0.4640 & 0.4683 & 0.4659 & 0.4693 & $\mathbf{0 . 4 7 7 1}$ \\
\hline
\end{tabular}

creases with lower FPS. We believe this happens because SSTS-GMSD also performs a temporal analysis. At the same time, the CSIQ, LIVE and IVP Databases have videos distorted by packetloss, which is a temporal distortion. Videos in the CSIQ and LIVE databases are in $768 \times 432$ and $832 \times 480$ spatial resolution, respectively, with $24,25,30,50$ and 60 FPS. Videos in the IVP and MCL-V databases are in 1088x1920 and 1080x1920 spatial resolution, respectively, with 25 and 30 FPS. Therefore, when we discard frames from videos in the CSIQ and LIVE databases, a lot more information is discarded.

For GMSD and SSIM, using videos sequences in 8 FPS is a reasonable trade-off between accuracy performance and runtime performance. The GMSD accuracy performance for the LIVE and MCL-V databases are better with videos sequence in 8 FPS than in the original FPS. For CSIQ and IVPL, the loss in accuracy performance is of $0.3 \%$ and $0.4 \%$, respectively, when comparing videos in the original FPS and videos in 8 FPS. The SSIM accuracy performance is best for videos in 8 FPS for LIVE, CSIQ, and IVP databases. For the IVP database, when we compare videos in the original FPS and videos in 8 FPS, the loss in accuracy performance is of $1.04 \%$. The best accuracy performance for PSNR is obtained for videos in 4 FPS for LIVE, CSIQ, and MCL-V database. For IVP database, the PSNR accuracy performance for videos in 4 FPS is $0.99 \%$ worse than for videos in the original FPS. 


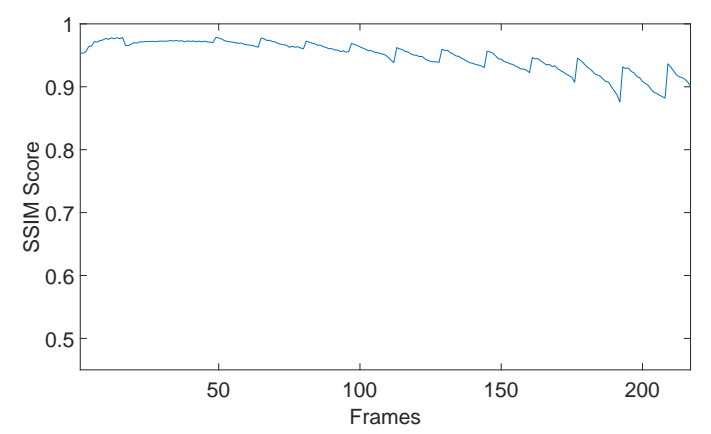

(a) H.264

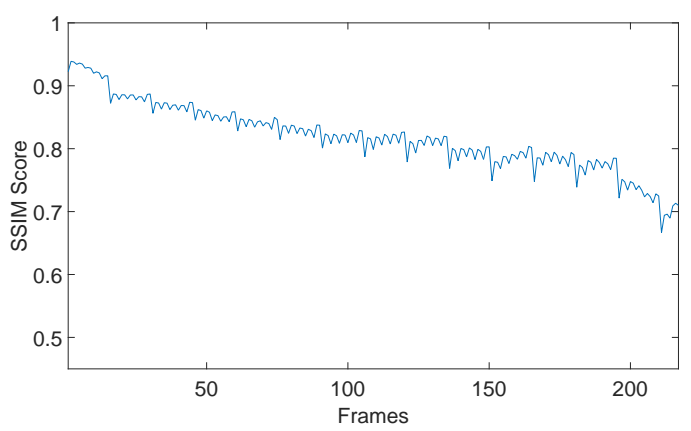

(b) MPEG-2

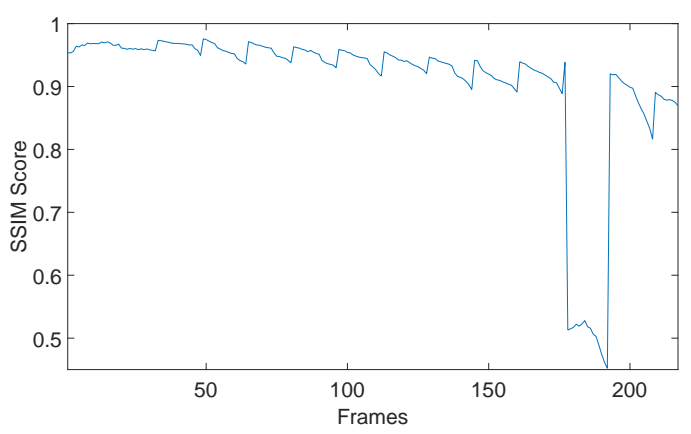

(c) Packet-Loss

Figure 5.3: SSIM score of 'Blue Sky' video distorted by H.264 Compression, MPEG-2 Compression and PacketLoss.

Although GMSD, SSIM, and PSNR are image quality metrics, they can be used to measure video quality by using them to measure the quality of each frame and, then, averaging the frame scores to obtain one overall video quality score. Figures 5.3 (a), (b), and (c) show the SSIM frame scores for a video sequence distorted by H.264 Compression, MPEG-2 compression, and PacketLoss, respectively. Notice that, for videos distorted by compression, neighboring frames have similar SSIM scores. SSIM frame scores for videos distorted by Packet-Loss have a different behavior. When a packet-loss occurs, the frame SSIM scores suddenly change for the frames where the distortion is located. Therefore, for temporal distortions, like packet-loss, discarding frames may reduce the accuracy of the quality metric more than reducing the size of the frames. In summary, unless the video has temporal distortions, it is fine to discard some frames when using GMSD, SSIM , and PSNR. Table 5.23 shows that, in most cases, the methods can perform best for packet-loss when using videos in the original temporal resolution or with 24 FPS.

\subsubsection{Discussion}

One of the challenges in image/video quality assessment is the computational time required by the quality metrics $[12,13]$. Notice that ViS3 and STRRED take more than three minutes, on average, to calculate the quality score of a video (see Table 5.18 ). Considering that all videos are, approximately, 10 seconds long, these methods cannot be used in real-time scenarios or practical applications. 
Table 5.23: Spearman Coefficient Correlation of the metrics when varying the video FPS for each video distortion.

\begin{tabular}{|c|c|c|c|c|c|c|}
\hline \multirow[t]{2}{*}{ Metric } & \multirow[t]{2}{*}{ Distortion } & \multicolumn{5}{|l|}{ FPS } \\
\hline & & Original & 24 & 16 & 8 & 4 \\
\hline \multirow{8}{*}{ ViS3 } & Wavelet & 0.6537 & 0.6329 & 0.6345 & 0.5772 & 0.5132 \\
\hline & H.264 & 0.7413 & 0.7479 & 0.7486 & 0.7481 & - \\
\hline & Н.265 & 0.9174 & 0.9236 & 0.9151 & 0.8878 & 0.8124 \\
\hline & MJPEG & 0.7349 & 0.7671 & 0.7560 & 0.6533 & 0.5613 \\
\hline & MPEG2 & 0.5278 & 0.5308 & 0.5239 & 0.5028 & 0.5140 \\
\hline & Upscaling & 0.6890 & 0.6927 & 0.7369 & 0.7200 & - \\
\hline & Packet-Loss & 0.8163 & 0.8128 & 0.7993 & 0.7643 & 0.7335 \\
\hline & White Noise & 0.9202 & 0.9156 & 0.9125 & 0.8826 & 0.8669 \\
\hline \multirow[t]{8}{*}{ STRRED } & Wavelet & 0.7825 & 0.7608 & 0.7579 & 0.7419 & 0.7303 \\
\hline & H.264 & 0.7718 & 0.7790 & 0.7768 & 0.7787 & 0.7605 \\
\hline & H.265 & 0.9135 & 0.9187 & 0.9230 & 0.9310 & 0.9212 \\
\hline & MJPEG & 0.7290 & 0.7223 & 0.7145 & 0.6857 & 0.6752 \\
\hline & MPEG2 & 0.6772 & 0.6615 & 0.6530 & 0.6289 & 0.6262 \\
\hline & Upscaling & 0.7040 & 0.7067 & 0.7006 & 0.6954 & 0.6902 \\
\hline & Packet-Loss & 0.8016 & 0.8067 & 0.8068 & 0.8014 & 0.7792 \\
\hline & White Noise & 0.9305 & 0.9377 & 0.9179 & 0.9192 & 0.9292 \\
\hline \multirow{8}{*}{$\begin{array}{l}\text { SSTS- } \\
\text { GMSD }\end{array}$} & Wavelet & 0.5981 & 0.5915 & 0.5783 & 0.5546 & 0.5345 \\
\hline & H.264 & 0.8025 & 0.7936 & 0.7921 & 0.7802 & 0.7861 \\
\hline & Н.265 & 0.9287 & 0.9359 & 0.9418 & 0.9375 & 0.9261 \\
\hline & MJPEG & 0.8803 & 0.8821 & 0.8736 & 0.8690 & 0.8396 \\
\hline & MPEG2 & 0.6272 & 0.6136 & 0.6062 & 0.6053 & 0.6181 \\
\hline & Upscaling & 0.6795 & 0.6806 & 0.6854 & 0.6936 & 0.6945 \\
\hline & Packet-Loss & 0.7899 & 0.7842 & 0.7797 & 0.7708 & 0.7516 \\
\hline & White Noise & 0.8819 & 0.8860 & 0.8811 & 0.8682 & 0.8456 \\
\hline \multirow[t]{8}{*}{ GMSD } & Wavelet & 0.5812 & 0.5798 & 0.5818 & 0.5805 & 0.5636 \\
\hline & H.264 & 0.7531 & 0.7517 & 0.7451 & 0.7640 & 0.7787 \\
\hline & H.265 & 0.9418 & 0.9441 & 0.9398 & 0.9416 & 0.9369 \\
\hline & MJPEG & 0.8842 & 0.8842 & 0.8847 & 0.8893 & 0.8744 \\
\hline & MPEG2 & 0.5501 & 0.5634 & 0.5371 & 0.5435 & 0.5569 \\
\hline & Upscaling & 0.6376 & 0.6378 & 0.6371 & 0.6400 & 0.6549 \\
\hline & Packet-Loss & 0.7770 & 0.7795 & 0.7708 & 0.7657 & 0.7172 \\
\hline & White Noise & 0.9094 & 0.9102 & 0.9102 & 0.9130 & 0.9066 \\
\hline \multirow[t]{8}{*}{ SSIM } & Wavelet & 0.6130 & 0.6141 & 0.6167 & 0.6106 & 0.6029 \\
\hline & Н.264 & 0.6257 & 0.6228 & 0.6223 & 0.6333 & 0.6464 \\
\hline & Н.265 & 0.8136 & 0.8139 & 0.8100 & 0.8124 & 0.8172 \\
\hline & MJPEG & 0.7969 & 0.7969 & 0.7969 & 0.8054 & 0.7974 \\
\hline & MPEG2 & 0.4814 & 0.4854 & 0.4825 & 0.4791 & 0.5224 \\
\hline & Upscaling & 0.4400 & 0.4408 & 0.4467 & 0.4406 & 0.4425 \\
\hline & Packet-Loss & 0.4485 & 0.4504 & 0.4433 & 0.4541 & 0.4166 \\
\hline & White Noise & 0.9300 & 0.9300 & 0.9282 & 0.9274 & 0.9300 \\
\hline \multirow[t]{8}{*}{ PSNR } & Wavelet & 0.6696 & 0.6716 & 0.6697 & 0.6711 & 0.6623 \\
\hline & H.264 & 0.6631 & 0.6616 & 0.6554 & 0.6714 & 0.6866 \\
\hline & H.265 & 0.7846 & 0.7938 & 0.7701 & 0.8015 & 0.7969 \\
\hline & MJPEG & 0.5086 & 0.5112 & 0.5086 & 0.5086 & 0.5341 \\
\hline & MPEG2 & 0.5371 & 0.5389 & 0.5282 & 0.5465 & 0.5475 \\
\hline & Upscaling & 0.4925 & 0.5015 & 0.4996 & 0.5045 & 0.5151 \\
\hline & Packet-Loss & 0.6509 & 0.6489 & 0.6401 & 0.6373 & 0.6252 \\
\hline & White Noise & 0.9063 & 0.9030 & 0.9063 & 0.9071 & 0.9009 \\
\hline
\end{tabular}


A simple method to reduce the computational time is to reduce the video spatial resolution. As seen in Tables 5.2 5.3, this strategy may decrease the accuracy performance of the methods. Since each database has a different set of distortions (see Tables 5.6- 5.9), when the spatial resolution is reduced, the methods may have different accuracy performances for each distortion. Therefore, we proposed two distortion classifiers to identify distortions that more or less sensitive to a reduction in spatial resolution. Tables 5.16 and 5.19 show the accuracy performance using these two classifiers. In both cases, the overall accuracy performance of the proposed framework is better than using the original resolution. For image quality assessment methods (PSNR, SSIM, and GMSD), the accuracy performance improvement is greater than for video quality assessment methods. Given that the accuracy performance when all videos are in $384 \times 216$ is worse, the distortion classifier is an important stage of the proposed framework.

Figures 5.4 and 5.5 show the runtime performance of the spatial activity classifier and the SSEQ classifier. The spatial activity classifier is a very fast method, allowing the framework to increase the speed of the original quality assessment methods. From the results of the accuracy performance analysis, we noticed that the framework with the spatial activity classifier improves both the computational and accuracy performances. But, since the SSEQ classifier requires extracting 12 features from each video frame, the framework with this classifier is slower. Nevertheless, it is still able to improve the runtime performance of the ViS3 and STRRED video quality assessment methods.

Finally, we analyze how a reduction in the temporal resolution affects the accuracy performance of quality metrics. For a video quality metric, which takes into account temporal information of the video, the accuracy performance is reduced when we decrease the temporal resolution of the input video. However, for image quality metrics, a reduction temporal resolution reduction does not affect the accuracy performance.

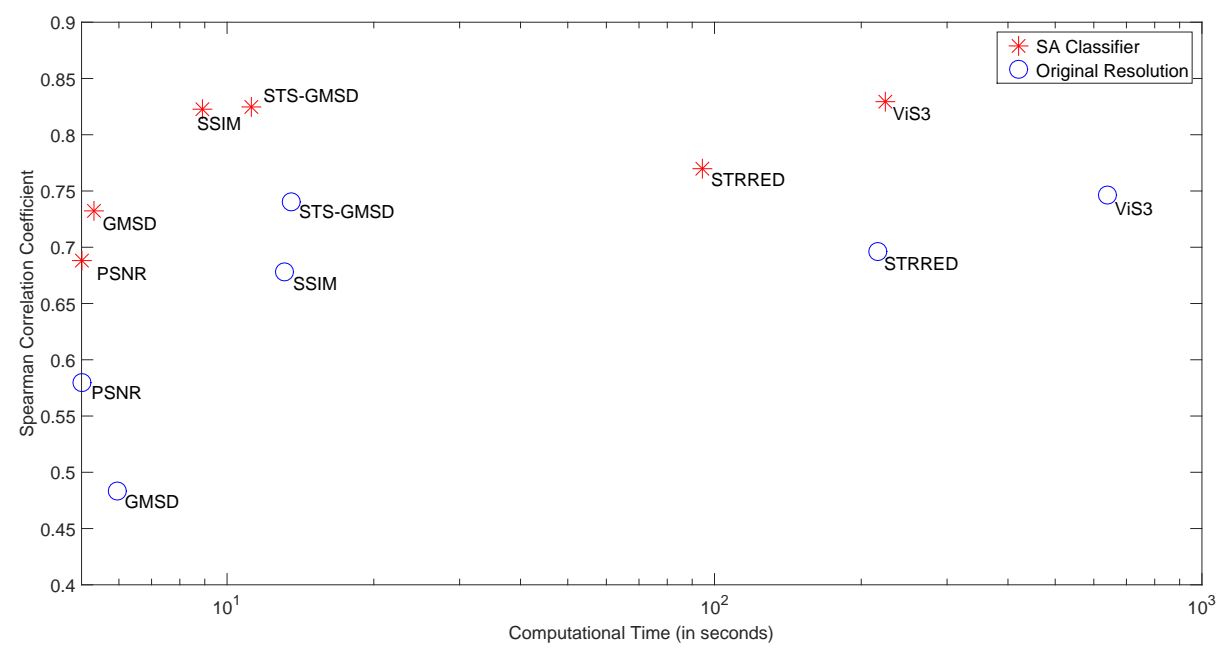

Figure 5.4: Spearman correlation coefficient versus computational time (in log space). Comparing the accuracy performance when using the Spatial Activity classifier and the video in their original resolution. 


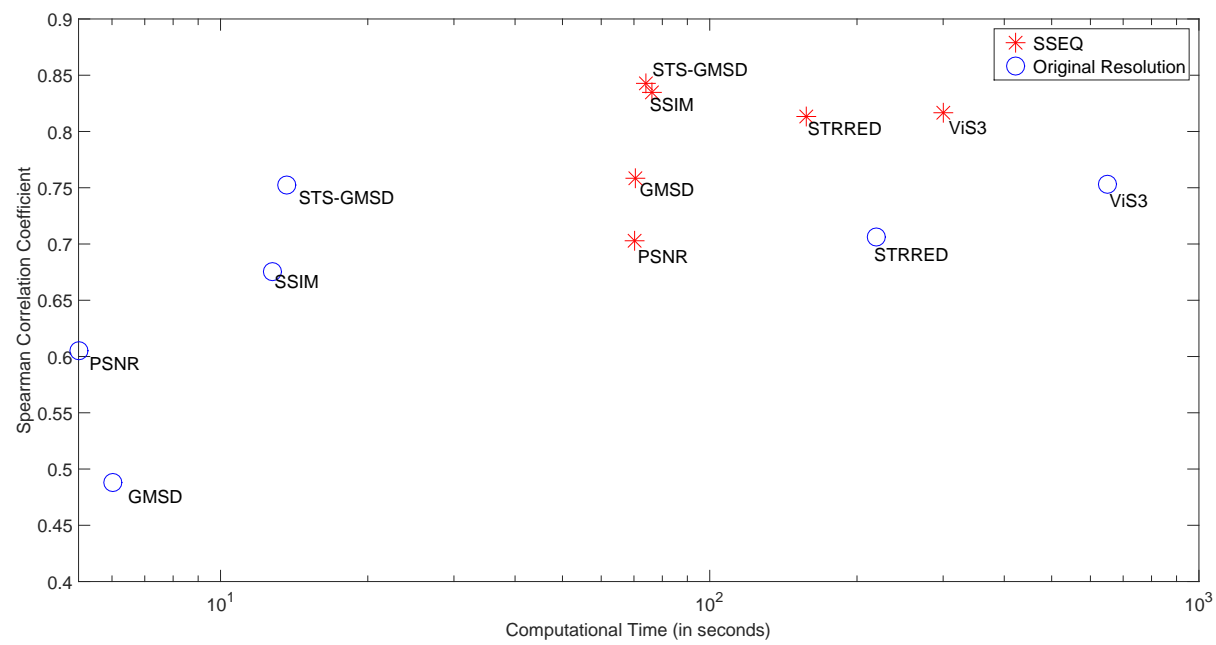

Figure 5.5: Spearman correlation coefficient versus computational time (in log space). Comparing the accuracy performance when using the SSEQ classifier and the video in their original resolution. 


\section{CONCLUSIONS AND FUTURE WORKS}

\subsection{CONCLUSIONS}

Over the last decades, several objective quality assessment methods have been design with the goal of estimating video quality. Unfortunately, these methods have become more complex and most of them cannot be used in real-time scenarios or in any practical application. For example, the ViS3 and STRRED metrics take minutes to estimate the quality of a video 10 seconds long. Therefore, one of the challenges in the area of video quality assessment is how to improve the runtime performance of currently available methods [12].

One of the fastest and simplest methods to improve the runtime performance of video processing algorithms is to reduce the spatial resolution of the video. In this work, we analyzed what happens to the accuracy performance of a set of video quality assessment methods when the spatial resolution is reduced. Six video quality assessment methods were used in this work: PSNR, SSIM, GMSD, SSTS-GMSD, STRRED, and ViS3. PSNR, SSIM, GMSD are image quality assessment methods that are frequently used to estimate video. SSTS-GMSD is a video quality assessment method based on the GMSD metric, STRRED is a video quality assessment method based on the entropic difference between the wavelet coefficients, and ViS3 is a video quality assessment method that computes the spatial distortions and the spatio-temporal dissimilarities between the reference and distorted video. These methods were chosen because each of them uses a different approach to evaluate quality.

The spatial resolution analysis showed that the method's accuracy performance is affected differently when the spatial resolution is reduced. Videos distorted by MJPEG compression, packet-loss and white noise are the most sensitive to the reduction of spatial resolution. In this work, we proposed a framework to improve the runtime of a given video quality metric, without decreasing its accuracy performance. This framework contains four stages: distortion classification, spatial downsampling of the input video (according to the distortion), quality estimation, and normalization of the predicted quality scores.

For the first stage, we proposed two classification methods to identify the videos that are more sensitive to a spatial resolution reduction. The first classification method is based on the spatial activity of the video. The performance of the framework using this classification method is very good, providing a fast and reliable quality estimate. The second classification method is based on spatial an spectral entropies. This method was originally developed as part of a quality assessment method [50]. We adapted it to work as a video distortion classification. Results using this classification method are also good, with the overall accuracy performance being sometimes better than what was obtained with the original quality metrics. Nevertheless, this classifications has a slow feature extraction process. As a consequence, this method only improves the speed of the STRRED and ViS3 metrics. 
We also performed an analysis of how temporal resolution affected the performance accuracy of quality assessment methods. We noticed that a loss in accuracy only for video quality assessment methods. Independently of the quality assessment method, the accuracy performance only decreased for videos distorted by packet-loss. Since packet-loss is a temporal distortion that may appear in any frame of the video, reducing the video temporal resolution may prevent the algorithm from detecting the packet-loss artifacts.

\subsection{FUTURE WORKS}

We studied the influence of the video distortion on the performance of video quality assessment methods when reducing the spatial resolution. When comparing the results for the spatial activity classifier and the ideal classification, we noticed that sometimes the spatial activity classifier gives better results. This means that it is not only the video distortion that influences the accuracy performance. Probably, the content of the videos also influences the method's performance. So, future works include analyzing the influence of the video content on the method's accuracy performance.

Another possible future work consists of analyzing the influence of reducing both spatial and temporal resolutions on the accuracy performance of quality assessment methods. To improve the proposed framework, it would be also important to find the optimal spatial and temporal resolutions. It is worth pointing out that, although temporal resolution is important for the video quality metrics, for the image quality metrics (e.g. GMSD, SSIM, and PSNR) a reduction of the temporal resolution does not affect the accuracy performance.

Finally, another interesting work would be to test our framework with a set of no-reference video quality assessment methods. In this case, the framework would require the use of a blind classifier, like the classifier of SSEQ.

\subsection{CONTRIBUTIONS}

Our final objective was to create a framework, that was able to reduce the run-time performance of video quality assessment methods. To achieve this objective, we did several analysis and proposed two video classifiers. Therefore, the main contributions of this work are:

- We analyzed the accuracy performance of six video quality metrics when the spatial video resolution was reduced. The tests were performed using four video quality databases.

- We analyzed an accuracy performance to identify which video distortions are more sensitive to a reduction in spatial resolution. For this analysis, we combined all databases and used the INSLA algorithm to normalize the database subjective scores. 
- We proposed two simple and fast video classifiers, which are able to identify which videos are more sensitive to a spatial resolution reduction.

- We analyzed the accuracy performance of the six video quality metrics for different temporal resolutions. Again, we used the same four video quality databases. We conclude that video quality assessment methods are more affected by temporal resolution reduction than image quality metrics.

- We analyzed an accuracy performance to identify which video distortions are more sensitive to a reduction in temporal resolution. For this analysis, we combined all databases and used the INSLA algorithm to normalize the database subjective scores.

- Our major contribution is our proposed framework, which is able to improve the runtime and accuracy performance of a given video quality assessment methods.

\subsection{PUBLISHED WORKS}

Journal Publications:

1. Freitas, Pedro Garcia, Welington YL Akamine, and Mylène CQ Farias. "Blind Image Quality Assessment Using Multiscale Local Binary Patterns." Journal of Imaging Science and Technology 60.6 (2016): 60405-1.

2. Akamine, Welington Yorihiko Lima; Farias, Mylène C. Q.. "Video quality assessment using visual attention computational models. Journal of Electronic Imaging (Print)", v. 23, p. 061107, 2014.

3. Farias, Mylene C.Q.; Akamine, Welington Yorihiko Lima . "On the Performance of Image Quality Metrics Enhanced with Visual Attention Computational Models". Electronics Letters, v. 48, p. 631-633, 2012.

Conference Publications:

1. Freitas, Pedro Garcia, Welington YL Akamine, and Mylène CQ Farias. "No-Reference Image Quality Assessment Using Texture Information Banks," In: 5th Brazilian Conference on Intelligent Systems (BRACIS), Recife, Brazil, 2016, pp. 127-132.

2. Freitas, Pedro Garcia, Welington YL Akamine, and Mylene CQ Farias. "No-reference image quality assessment based on statistics of Local Ternary Pattern." In: Quality of Multimedia Experience (QoMEX), 2016 Eighth International Conference on. IEEE, 2016.

3. Akamine, Welington Yorihiko Lima ; Farias, Mylène C. Q.; "Incorporating visual attention models into video quality metrics", In: Image Quality and System Performance XI, IST/SPIE Electronic Imaging, v. 9016, 2014, San Francisco. 
4. Akamine, Welington Yorihiko Lima; Farias, Mylène C.Q.. "The Added Value of Visual Attention in Objective Video Quality Metrics”, In: Eighth International Workshop on Video Processing and Quality Metrics for Consumer Electronics, 2014, Chandler, AZ.

5. Daniele Vieira ; Akamine, Welington Yorihiko Lima ; RODRIGUES, G. ; Farias, Mylène C. Q. . "Um Estudo Sobre o Impacto da Atenção Visual nas Redes Sociais”, In: II Brazilian Workshop on Social Network Analysis and Mining, 2013, Maceió, BraSNAM 2013. p. 1.

6. FARIAS, Mylene Christine Queiroz de ; Akamine, Welington Yorihiko Lima. "Incorporating Visual Attention Models into Image Quality Metric", In: Sixth International Workshop on Video Processing and Quality Metrics for Consumer Electronics, Scottsdale, Arizona, EUA, 2012.

7. Akamine, Welington Yorihiko Lima ; Farias, Mylene C.Q. "Studying The Added Value of Visual Attention in Objective Image Quality Metrics", In: Workshop of Undergraduate Works, SIBGRAPI 2012 - Conference on Graphics, Patterns and Images, 2012, Ouro Preto, MG. 


\section{Bibliography}

1 WANG, Z.; BOVIK, A. C.; SHEIKH, H. R.; SIMONCELLI, E. P. Image quality assessment: from error visibility to structural similarity. IEEE Transactions on Image Processing, v. 13, n. 4, p. 600-612, April 2004. ISSN 1057-7149.

2 YAN, P.; MOU, X.; XUE, W. Video quality assessment via gradient magnitude similarity deviation of spatial and spatiotemporal slices. Proc. SPIE, v. 9411, p. 94110M-94110M-10, 2015. Disponível em: $<$ http://dx.doi.org/10.1117/12.2083283>.

3 SIMONCELLI, E. P.; FREEMAN, W. T. The steerable pyramid: A flexible architecture for multi-scale derivative computation. In: IEEE. Image Processing, 1995. Proceedings., International Conference on. [S.1.], 1995. v. 3, p. 444-447.

4 VU, P. V.; CHANDLER, D. M. Vis3: an algorithm for video quality assessment via analysis of spatial and spatiotemporal slices. Journal of Electronic Imaging, v. 23, n. 1, p. 013016, 2014. Disponível em: $<$ http://dx.doi.org/10.1117/1.JEI.23.1.013016>.

5 CISCO Visual Networking Index: Forecast and Methodology, 2014-2019 White Paper. 2015.

6 SESHADRINATHAN, K.; SOUNDARARAJAN, R.; BOVIK, A. C.; CORMACK, L. K. Study of subjective and objective quality assessment of video. IEEE transactions on image processing, IEEE, v. 19, n. 6, p. 1427-1441, 2010.

7 WANG, Z.; BOVIK, A. C. Mean squared error: Love it or leave it? a new look at signal fidelity measures. IEEE Signal Processing Magazine, v. 26, n. 1, p. 98-117, Jan 2009. ISSN 1053-5888.

8 WANG, Z.; LU, L.; BOVIK, A. C. Video quality assessment based on structural distortion measurement. Signal processing: Image communication, Elsevier, v. 19, n. 2, p. 121-132, 2004.

9 VU, P. V.; VU, C. T.; CHANDLER, D. M. A spatiotemporal most-apparent-distortion model for video quality assessment. In: IEEE. 2011 18th IEEE International Conference on Image Processing. [S.1.], 2011. p. 2505-2508.

10 PINSON, M. H.; WOLF, S. A new standardized method for objectively measuring video quality. IEEE Transactions on broadcasting, IEEE, v. 50, n. 3, p. 312-322, 2004.

11 SESHADRINATHAN, K.; BOVIK, A. C. Motion tuned spatio-temporal quality assessment of natural videos. IEEE transactions on image processing, IEEE, v. 19, n. 2, p. 335-350, 2010.

12 CHANDLER, D. M. Seven challenges in image quality assessment: past, present, and future research. ISRN Signal Processing, Hindawi Publishing Corporation, v. 2013, 2013.

13 WANG, Z. Objective image quality assessment: Facing the real-world challenges. Electronic Imaging, Society for Imaging Science and Technology, v. 2016, n. 13, p. 1-6, 2016.

14 FARIAS, M. C. Video quality metrics. [S.1.]: INTECH Open Access Publisher, 2010.

15 SAYOOD, K. Introduction to Data Compression. [S.1.]: Morgan Kaufmann, 2012. (Morgan Kaufmann series in multimedia information and systems). ISBN 9780124157965.

16 AMER, A.; MITICHE, A.; DUBOIS, E. Reliable and fast structure-oriented video noise estimation. In: IEEE. Image Processing. 2002. Proceedings. 2002 International Conference on. [S.1.], 2002. v. 1, p. I-840. 
17 RISSANEN, J. Modeling by shortest data description. Automatica, Elsevier, v. 14, n. 5, p. 465-471, 1978.

18 PENNEBAKER, W. B.; MITCHELL, J. L. JPEG: Still image data compression standard. [S.1.]: Springer Science \& Business Media, 1992.

19 UNTERWEGER, A. Compression artifacts in modern video coding and state-of-the-art means of compensation. Multimedia Networking and Coding, IGI Global, p. 28, 2012.

20 GONZALEZ, R. C.; WOODS, R. E. Digital Image Processing (3rd Edition). Upper Saddle River, NJ, USA: Prentice-Hall, Inc., 2006. ISBN 013168728X.

21 TUDOR, P. Mpeg-2 video compression. Electronics \& communication engineering journal, IET, v. 7, n. 6, p. 257-264, 1995.

22 WIEGAND, T.; SULLIVAN, G. J.; BJONTEGAARD, G.; LUTHRA, A. Overview of the h. 264/avc video coding standard. IEEE Transactions on circuits and systems for video technology, IEEE, v. 13, n. 7, p. 560-576, 2003.

23 KOH, C. C.; MITRA, S. K.; FOLEY, J. M.; HEYNDERICKX, I. E. Annoyance of individual artifacts in mpeg-2 compressed video and their relation to overall annoyance. In: INTERNATIONAL SOCIETY FOR OPTICS AND PHOTONICS. Electronic Imaging 2005. [S.1.], 2005. p. 595-606.

24 LIST, P.; JOCH, A.; LAINEMA, J.; BJONTEGAARD, G.; KARCZEWICZ, M. Adaptive deblocking filter. IEEE transactions on circuits and systems for video technology, v. 13, n. 7, p. 614-619, 2003.

25 BORER, T.; DAVIES, T. Dirac video compression using open technology. BBC EBU technical review, 2005.

26 SULLIVAN, G. J.; OHM, J.-R.; HAN, W.-J.; WIEGAND, T. Overview of the high efficiency video coding (hevc) standard. IEEE Transactions on circuits and systems for video technology, IEEE, v. 22, n. 12, p. 1649-1668, 2012.

27 OHM, J.-R.; SULLIVAN, G. J.; SCHWARZ, H.; TAN, T. K.; WIEGAND, T. Comparison of the coding efficiency of video coding standards-including high efficiency video coding (hevc). IEEE Transactions on Circuits and Systems for Video Technology, IEEE, v. 22, n. 12, p. 1669-1684, 2012.

28 LIN, J. Y.; SONG, R.; WU, C.-H.; LIU, T.; WANG, H.; KUO, C.-C. J. Mcl-v: A streaming video quality assessment database. Journal of Visual Communication and Image Representation, Elsevier, v. 30, p. 1-9, 2015.

29 PARKER, J. A.; KENYON, R. V.; TROXEL, D. E. Comparison of interpolating methods for image resampling. IEEE Transactions on medical imaging, IEEE, v. 2, n. 1, p. 31-39, 1983.

30 BOULOS, F.; PARREIN, B.; CALLET, P. L.; HANDS, D. Perceptual effects of packet loss on h. 264/avc encoded videos. In: Fourth International Workshop on Video Processing and Quality Metrics for Consumer Electronics VPQM-09. [S.1.: s.n.], 2009.

31 SESHADRINATHAN, K.; SOUNDARARAJAN, R.; BOVIK, A. C.; CORMACK, L. K. A subjective study to evaluate video quality assessment algorithms. In: INTERNATIONAL SOCIETY FOR OPTICS AND PHOTONICS. IS\&T/SPIE Electronic Imaging. [S.1.], 2010. p. 75270H-75270H.

32 BT.500-11, I.-R. R. Methodology for the subjective assessment of the quality of television pictures. v. 2002. Disponível em: <https://www.itu.int/>.

33 WINKLER, S.; MOHANDAS, P. The evolution of video quality measurement: From psnr to hybrid metrics. IEEE Transactions on Broadcasting, v. 54, n. 3, p. 660-668, Sept 2008. ISSN 0018-9316. 
34 LUBIN, J.; FIBUSH, D. Sarnoff JND vision model. [S.1.]: T1A1, 1997.

35 WINKLER, S. Perceptual distortion metric for digital color video. In: INTERNATIONAL SOCIETY FOR OPTICS AND PHOTONICS. Electronic Imaging'99. [S.1.], 1999. p. 175-184.

36 XUE, W.; ZHANG, L.; MOU, X.; BOVIK, A. C. Gradient magnitude similarity deviation: a highly efficient perceptual image quality index. IEEE Transactions on Image Processing, IEEE, v. 23, n. 2, p. 684-695, 2014.

37 LARSON, E. C.; CHANDLER, D. M. Most apparent distortion: full-reference image quality assessment and the role of strategy. Journal of Electronic Imaging, International Society for Optics and Photonics, v. 19, n. 1, p. 011006-011006, 2010.

38 XU, J.; YE, P.; LIU, Y.; DOERMANN, D. No-reference video quality assessment via feature learning. In: IEEE. 2014 IEEE International Conference on Image Processing (ICIP). [S.1.], 2014. p. 491-495.

39 SAAD, M. A.; BOVIK, A. C.; CHARRIER, C. Blind prediction of natural video quality. IEEE Transactions on Image Processing, IEEE, v. 23, n. 3, p. 1352-1365, 2014.

40 SOUNDARARAJAN, R.; BOVIK, A. C. Video quality assessment by reduced reference spatiotemporal entropic differencing. IEEE Transactions on Circuits and Systems for Video Technology, v. 23, n. 4, p. 684-694, April 2013. ISSN 1051-8215.

41 SOUNDARARAJAN, R.; BOVIK, A. C. Rred indices: Reduced reference entropic differencing for image quality assessment. IEEE Transactions on Image Processing, IEEE, v. 21, n. 2, p. 517-526, 2012.

42 WAINWRIGHT, M. J.; SIMONCELLI, E. P. Scale mixtures of gaussians and the statistics of natural images. p. 855-861, 1999.

43 LUCAS, B. D.; KANADE, T. et al. An iterative image registration technique with an application to stereo vision. In: IJCAI. [S.1.: s.n.], 1981. v. 81, n. 1, p. 674-679.

44 SESHADRINATHAN, K.; SOUNDARARAJAN, R.; BOVIK, A. C.; CORMACK, L. K. Study of subjective and objective quality assessment of video. IEEE Transactions on Image Processing, v. 19, n. 6, p. 1427-1441, June 2010. ISSN 1057-7149.

45 KOZAMERNIK, F.; SUNNA, P.; WYCKENS, E.; PETTERSEN, D. I. Subjective quality of internet video codecs phase ii evaluations using samviq. EBU technical Review, v. 301, 2005.

46 ZHANG, F.; LI, S.; MA, L.; WONG, Y. C.; NGAN, K. N. IVP subjective quality video database. 2011. Disponível em: <http://ivp.ee.cuhk.edu.hk/research/database/subjective/>.

47 RECOMMENDATION, P. I.-T. Subjective video quality assessment methods for multimedia applications. 1999.

48 FENIMORE, C.; LIBERT, J.; WOLF, S. Perceptual effects of noise in digital video compression. SMPTE journal, SMPTE, v. 109, n. 3, p. 178-187, 2000.

49 BORER, S.; LESZCUK, M. MOAVI indicators. Disponível em: <http://vq.kt.agh.edu.pl//metrics. html>.

50 LIU, L.; LIU, B.; HUANG, H.; BOVIK, A. C. No-reference image quality assessment based on spatial and spectral entropies. Signal Processing: Image Communication, v. 29, n. 8, p. 856 - 863, 2014. ISSN 0923-5965. Disponível em: <http://www.sciencedirect.com/science/article/pii/S0923596514000927>.

51 MOORTHY, A. K.; BOVIK, A. C. A two-step framework for constructing blind image quality indices. IEEE Signal Processing Letters, v. 17, n. 5, p. 513-516, May 2010. ISSN 1070-9908. 
52 AHMED, N.; NATARAJAN, T.; RAO, K. R. Discrete cosine transform. IEEE Transactions on Computers, C-23, n. 1, p. 90-93, Jan 1974. ISSN 0018-9340.

53 CORTES, C.; VAPNIK, V. Support-vector networks. Machine learning, Springer, v. 20, n. 3, p. 273-297, 1995.

54 HSU, C.-W.; LIN, C.-J. A comparison of methods for multiclass support vector machines. IEEE transactions on Neural Networks, IEEE, v. 13, n. 2, p. 415-425, 2002.

55 SCHULDT, C.; LAPTEV, I.; CAPUTO, B. Recognizing human actions: A local svm approach. In: IEEE. Pattern Recognition, 2004. ICPR 2004. Proceedings of the 17th International Conference on. [S.1.], 2004. v. 3, p. 32-36.

56 LIN, W.-H.; HAUPTMANN, A. News video classification using svm-based multimodal classifiers and combination strategies. In: ACM. Proceedings of the tenth ACM international conference on Multimedia. [S.1.], 2002. p. 323-326.

57 MICHEL, P.; KALIOUBY, R. E. Real time facial expression recognition in video using support vector machines. In: ACM. Proceedings of the 5th international conference on Multimodal interfaces. [S.1.], 2003. p. 258-264.

58 KEYS, R. Cubic convolution interpolation for digital image processing. IEEE Transactions on Acoustics, Speech, and Signal Processing, v. 29, n. 6, p. 1153-1160, Dec 1981. ISSN 0096-3518.

59 GROUP, V. Q. E. et al. Final report from the video quality experts group on the validation of objective models of video quality assessment, phase ii (fr_tv2). 2003.

60 PINSON, M. H.; WOLF, S. An objective method for combining multiple subjective data sets. Proc. SPIE, v. 5150, p. 583-592, 2003. Disponível em: <http://dx.doi.org/10.1117/12.509909>.

61 SHEIKH, H. R.; SABIR, M. F.; BOVIK, A. C. A statistical evaluation of recent full reference image quality assessment algorithms. IEEE Transactions on image processing, IEEE, v. 15, n. 11, p. 3440-3451, 2006.

62 MANNING, C. D.; RAGHAVAN, P.; SCHüTZE, H. Introduction to Information Retrieval. New York, NY, USA: Cambridge University Press, 2008. ISBN 0521865719, 9780521865715.

63 SPEARMAN, C. The proof and measurement of association between two things. The American journal of psychology, JSTOR, v. 15, n. 1, p. 72-101, 1904. 
APPENDIX 


\section{EVALUTUATION MEASURES}

To evaluate classification methods is common to use the recall, precision an F1-Score [62]. The results of a classification method can be represented in a confusion matrix. The example of a confusion matrix in a binary classification is shown in Table I.1.

Table I.1: Example of a Confusion Matrix

\begin{tabular}{l|l|l|} 
& \multicolumn{1}{l}{ Real Positive } & \multicolumn{1}{l}{ Real Negative } \\
\cline { 2 - 3 } Predicted Positive & True Positive & False Positive \\
\cline { 2 - 3 } Predicted Negative & False Negative & True Negative \\
\cline { 2 - 3 } & &
\end{tabular}

The confusion matrix represents all the results of a classifier into a single table. Predicted Positive are the cases, which the method classify as positive. Predicted Negative are the cases, which the method classify as negative. Real Positive are the cases that are labeled as positive. And Real Negative are the cases labeled as negative. The true positive are the cases which the classifier correctly predicted as positive or negative cases, respectively. The false positive and false negative are the cases which the classifier wrongly predicted as positive or negative cases, respectively.

\section{I.1 RECALL}

Recall measures the proportion of the real positive cases correctly classify by the classification method. It is most relevant in applications that aim to identify real positive cases. Recall is defined by:

$$
\text { Recall }=\frac{\text { True Positive }}{\text { Real Positive }}
$$

\section{I.2 PRECISON}

Precision measures the proportion of the predicted positive cases correctly classify by the classification method. It is a measure of the true positive accuracy. Precision is define by:

$$
\text { Precision }=\frac{\text { True Positive }}{\text { Predicted Positive }}
$$




\section{I.3 F1-SCORE}

F-Measure is a single measure that combines the results of precision and recall. F-Measure is a harmonic mean of precision and recall, define by:

$$
F-\text { Measure }=\frac{1}{\alpha \frac{1}{\text { Precision }}+(1-\alpha) \frac{1}{\text { Recall }}}=\frac{\left(\beta^{2}+1\right) \text { Precision } \cdot \text { Recall }}{\beta^{2} \text { Precision }+ \text { Recall }}
$$

where $\beta^{2}=\frac{1-\alpha}{\alpha}, \beta$ is a parameter that controls the balance of precision and recall. F1 Score is the special case when $\beta=1$, in other words, the F-Measure equally weights precision and recall. F1 Score is defined by:

$$
F 1=\frac{2 \cdot \text { Precision } \cdot \text { Recall }}{\text { Precision }+ \text { Recall }}
$$




\section{SPEARMAN CORRELATION}

The Spearman Correlation was proposed by Spearman in 1904 [63]. It measures the prediction monotonicity of a model. In this work, we use the Spearman Correlation to compare the prediction quality scores, given by the video quality assessment methods, with the subjective quality scores. Let $x_{i}$ denote the predict score and $y_{i}$ denote the subjective score, where $i \in 1,2, \ldots, M$. The Spearman Correlation Coefficient SCC is defined by the following equation:

$$
S C C=\frac{\sum_{i=1}^{M}\left(\chi_{i}-\bar{\chi}\right)\left(\gamma_{i}-\bar{\gamma}\right)}{\sqrt{\sum_{i=1}^{M}\left(\chi_{i}-\bar{\chi}\right)^{2}} \sqrt{\sum_{i=1}^{M}\left(\gamma_{i}-\bar{\gamma}\right)^{2}}}
$$

where $\chi_{i}$ is the rank of $x_{i}$ and $\gamma_{i}$ is the rank of $y_{i}$ in the ordered data series, $\bar{\chi}$ and $\bar{\gamma}$ are the respect midrank. The SCC makes no assumption about the shape of the relationship between $x_{i}$ and $y_{i}$. 